\title{
PERFORMANCE OF CONCATENATED WALSH/PN SPREADING SEQUENCES FOR CDMA SYSTEMS
}

\author{
By \\ Johnson Oghenevwareh Sebeni
}

B. Eng. (Electrical and Electronics Engineering), University of Benin, Nigeria, 1989

\author{
A THESIS SUBMITTED IN PARTIAL FULFILLMENT OF \\ THE REQUIREMENTS FOR THE DEGREE OF \\ MASTER OF APPLIED SCIENCE \\ in \\ THE FACULTY OF GRADUATE STUDIES
}

DEPARTMENT OF ELECTRICAL AND COMPUTER ENGINEERING

We accept this thesis as conforming to the required standard

THE UNIVERSITY OF BRITISH COLUMBIA

August 1998

(C) Johnson Oghenevwareh Sebeni, 1998 
In presenting this thesis in partial fulfilment of the requirements for an advanced degree at the University of British Columbia, I agree that the Library shall make it freely available for reference and study. I further agree that permission for extensive copying of this thesis for scholarly purposes may be granted by the head of my department or by his or her representatives. It is understood that copying or publication of this thesis for financial gain shall not be allowed without my written permission.

Department of Electrical and Computer Engineering The University of British Columbia

2075 Wesbrook Place

Vancouver, Canada

V6T 1W5

Date:

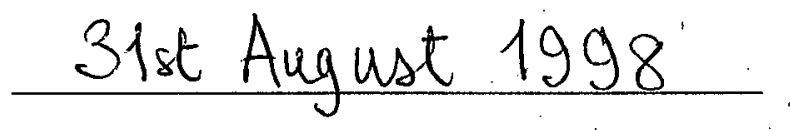




\begin{abstract}
The Bit Error Rate (BER) performance on the forward link of a Code Division Multiple Access (CDMA) system using the concatenated orthogonal Walsh/m-sequence scheme for bandwidth spreading is studied. Expressions are derived for the BER for single cell systems using the Gaussian approximation and the characteristic function method. Performance results obtained using these expressions are compared to those obtained by Monte Carlo simulation. It was found that BER estimates using the Gaussian approximation are inaccurate because of statistical dependence among the interference terms.

To take advantage of multipath diversity, the performance of the Rake receiver is compared to that of the correlation receiver for such systems. A modified Rake receiver is suggested whose BER performance is shown to be better than that of the Rake and correlation receiver especially for Rician fading multipath channels with Rice factor greater than $2 \mathrm{~dB}$.

The area-averaged BER on the forward link of a multiple cell CDMA system is evaluated. A lognormal probability density function (pdf) is suggested to approximate the distribution of the ratio of the sum of average power of the received signals from other base stations to that of the received signal from the controlling base station which is assumed to be the one with the lowest attenuation to the mobile. A comparison of areaaveraged BER's obtained using this approximate pdf and the Gaussian approximation to those obtained by Monte Carlo simulation shows a reasonable agreement.
\end{abstract}




\section{Table of Contents}

Abstract $\quad$ ii

List of Figures $\quad$ vi

$\begin{array}{ll}\text { Acknowledgments } & \text { ix }\end{array}$

1 Introduction $\quad 1$

2 Background $\quad 3$

2.1 Parameters of Spreading Codes ... . . . . . . . . . . . . 3

2.1.1 System and Channel Model ............... 4

2.1.2 Autocorrelation parameters ............. 5

2.1.3 Cross-correlation parameters .............. 8

2.1.4 System Performance ........................... 10

2.2 Concatenated Orthogonal/ PN Spreading Code . . . . . . . . . 13

2.2.1 A Multi-Code CDMA System ............... . 14

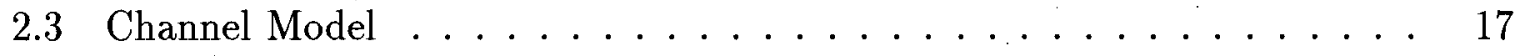

2.3.1 Statistics of Fading . . . . . . . . . . . . 18

2.3.2 Statistics of Phase Distortion . . . . . . . . . . . 19

2.3.3 Statistics of Transmission Delays Through Multipaths . . . . . . 19

2.4 Multipath Diversity . . . . . . . . . . . . . . . 20

2.4.1 Selection Combining Technique . . . . . . . . . . 21

2.4.2 Maximal-Ratio Combining Technique . . . . . . . . . . 22 
3 Performance of Concatenated CDMA codes for Forward Links

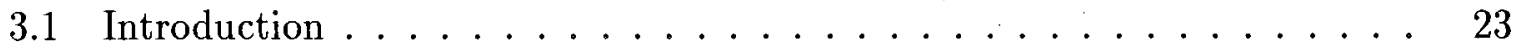

3.2 System Model . . . . . . . . . . . . . . . . . . . . . . 24

3.3 Channel Model ......................... 25

3.4 Channels with zeroth path having Rayleigh distributed fading . . . . . . 27

3.4.1 Evaluation of BER Via the Characteristic Function Method .. . . 27

3.4.2 Evaluation of Bit Error Probability Via the Gaussian Approximation of the Multipath Interference . . . . . . . . . . . 30

3.4.3 Evaluation of Bit Error Probability Via Monte Carlo Simulation 32

3.5 Channels with zeroth path having Rician distributed fading . . . . . . 33

3.5.1 Evaluation of Bit Error Probability Via the Characteristic Function

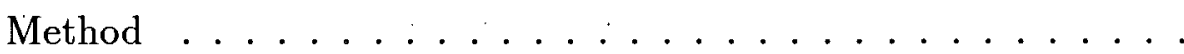

3.5.2 Evaluation of Bit Error Probability Via the Gaussian Approximation of the Multiple Access Interference . . . . . . . . . . . . 34

3.5.3 Evaluation of Bit Error Probability Via Monte Carlo Simulation . 35

3.6 Numerical Results . . . . . . . . . . . . . . . . 35

4 Multipath Diversity $\quad 52$

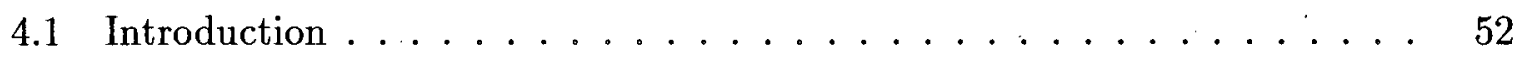

4.2 System and Channel Model . . . . . . . . . . . . . . . 53

4.3 BER for channels with Rayleigh fading on the zeroth path . . . . . . . . 54

4.3.1 Gaussian approximation ................. 56

4.3.2 BER via Monte Carlo Simulation . . . . . . . . . . . . 59

4.4 BER for channels with Rician fading on the zeroth path . . . . . . . . 59

4.4.1 Gaussian Approximation ................ 60

4.4.2 BER via Monte Carlo Simulation . . . . . . . . . . . 61 
4.5 A Modified Diversity Receiver for Environments with weak multipath signals 61

4.6 Numerical Results . . . . . . . . . . . . . . . . . . 62

5 Performance on the Forward Link in a Multicell Environment $\quad 73$

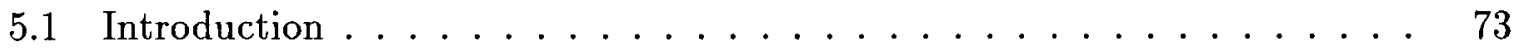

5.2 System and Channel Model . . . . . . . . . . . . . . . . . 74

5.3 BER for Rayleigh faded channels . . . . . . . . . . . 76

5.4 First and Second Moments of $y \ldots \ldots \ldots$. . . . . . . . . . . . . .

5.5 BER for Rician faded channels . . . . . . . . . . . . . 83

5.6 Numerical Results . . . . . . . . . . . . . . . . 85

6 Conclusion and Further Study $\quad 92$

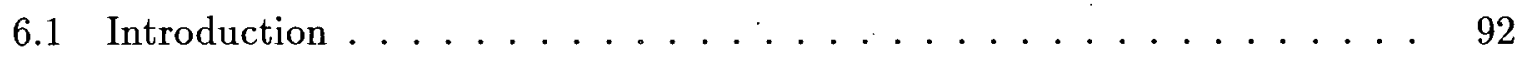

6.2 Contributions of the Thesis and Suggestions for Further Study . . . . . 92

$\begin{array}{ll}\text { Glossary } & 96\end{array}$

$\begin{array}{ll}\text { Bibliography } & 97\end{array}$ 


\section{List of Figures}

2.1 A transmitter structure for Multi-Code CDMA $\ldots \ldots \ldots \ldots$

3.1 Generation of the concatenated Walsh $/$ m-sequence $\ldots \ldots \ldots 25$

3.2 BER in a Rayleigh faded channel with weak multipaths, $M=64 \ldots \ldots 41$

3.3 BER in a Rayleigh faded channel with strong multipaths, $M=64 \ldots 42$

3.4 BER in a Rayleigh faded channel (with weak paths) using different evaluation techniques, $M=64 \ldots \ldots \ldots \ldots \ldots \ldots$

3.5 BER in a Rayleigh faded channel (with strong paths) using different evaluation techniques, $M=64 \ldots \ldots \ldots \ldots \ldots \ldots$

3.6 BER in a Rician faded channel with weak multipaths, $M=64 \ldots \ldots 45$

3.7 BER in a Rician faded channel with strong multipaths, $M=64 \ldots \ldots$

3.8 BER in a Rician faded channel (with weak paths) using different evaluation techniques, $M=64 \ldots \ldots \ldots \ldots \ldots \ldots \ldots \ldots$

3.9 BER in a Rician faded channel (with weak paths and $10 \%$ fading power on the zeroth path) using different evaluation techniques, $M=64 \ldots$.

3.10 BER in a Rician faded channel (with strong paths) using different evaluation techniques, $M=64 \ldots \ldots \ldots \ldots \ldots \ldots \ldots \ldots \ldots \ldots$

3.11 Probability density function of the multipath interference (weak multipaths) when $\beta=1.0,\left|\alpha_{1}\right|=\gamma_{1},\left|\alpha_{2}\right|=\gamma_{2}$ and $\gamma_{0}=0 \ldots \ldots \ldots$

3.12 BER in a Rician faded channel (with weak paths) using different evaluation techniques, $M=64 \ldots \ldots \ldots \ldots \ldots \ldots \ldots \ldots \ldots \ldots \ldots \ldots$

4.1 Rake Receiver (delayed received signal configuration) $\ldots \ldots \ldots$. . . . . 54 
4.2 Bits affected by multipath interference in three path channel . . . . . 55

4.3 Modified Rake Receiver . . . . . . . . . . . . . . . . 63

4.4 BER in a Rayleigh faded channel with weak multipaths, $M=64, P=$ 2047 (correlation receiver, Rake receiver and modified Rake receiver) . .

4.5 BER in a Rayleigh faded channel with strong multipaths, $M=64, P=$ 2047 (correlation receiver, Rake receiver and modified Rake receiver) . .

4.6 BER in a Rician faded channel with weak multipaths, $M=64, P=2047$ (correlation receiver, Rake receiver and modified Rake receiver) . . . .

4.7 BER in a Rician faded channel with weak multipaths and $10 \%$ fading power on the zeroth path, $M=64, P=2047$ (Rake receiver only) . . .

4.8 BER in a Rician faded channel with strong multipaths, $M=64, P=2047$ (correlation receiver, Rake receiver and modified Rake receiver) . . . . .

4.9 BER in a Rician faded channel (with different Rice Factor $K_{r}$ ) using Rake receiver, correlation receiver and the modified Rake receivers; $M=64$, $P=2047$ and Number of users $K=64 \ldots \ldots \ldots$

5.1 Probability density function of the the ratio of the sum of the average received power from the other base stations to the average received power from the controlling base station . . . . . . . . . . . . . .

5.2 Area-averaged BER in a Rayleigh faded channel with weak multipaths, $M=64, P=2047$ (Rake receiver) . . . . . . . . . .

5.3 Area-averaged BER in a Rayleigh faded channel with strong multipaths, $M=64, P=2047$ (Rake receiver) . . . . . . . . . .

5.4 Area-averaged BER in a Rician faded channel with weak multipaths, $M=$ $64, P=2047$ (Rake receiver) $\ldots \ldots \ldots \ldots$ 
5.5 Area-averaged BER in a Rician faded channel with strong multipaths, $M=64, P=2047$ (Rake receiver) $\ldots \ldots \ldots$. . . . . . . 91 


\section{Acknowledgments}

First, I would like to thank my thesis supervisor, Dr. Cyril Leung, for his guidance throughout the duration of this thesis work. His constructive criticism, useful suggestions and encouragements gave me the needed strength to complete this project. I would also like to thank Mr Ian Marsland and other people in the Communications Group for their support and help. This work was supported by the African Development Bank (ADB) and a Government of Japan fellowship grant ADB/JAP/96/0251. I owe the ADB and the Government of Japan a debt of gratitude for giving me the opportunity to pursue my studies at the University of British Columbia. Finally, I would like to thank my parents, Mr. Paul Sebeni and Mrs Victoria Sebeni, for their understanding throughout the duration of this program. 


\section{Chapter 1}

\section{Introduction}

In the last few years, wireless communications has undergone a rapid growth in its commercial deployment which has led to new visions for its applications. From the present available applications in mobile telephony, there are numerous future applications in the area of multimedia communications. Also, there has been a big leap in the number of subscribers using wireless means for communication. The implication of this large number of users and the planned future applications is the need to design wireless systems that can accommodate more users and meet their respective quality of service requirements. In addition, such systems should be able to share the same limited communication resources amongst the different applications with the feature that unused resources by one application can easily be used by other applications, without the need for very expensive subscriber units.

Important to CDMA systems is the use of bandwidth spreading codes which can lead to more efficient sharing of limited communication resources. Several spreading codes such as Pseudo-noise (PN) codes and the set of Walsh sequences have been studied for use in CDMA systems $[1,2]$. In $[3,4]$, a concatenated Walsh/m-sequence scheme was studied for application as a spreading code in the forward link of cellular CDMA systems. In this thesis, this type of spreading code is further studied. In addition to evaluating the bit error probability performance of the forward link of such systems, different bit error probability performance evaluation techniques are compared.

In Chapter 2, we review some of the fundamental concepts used in CDMA systems 
that will serve as background material throughout the thesis. We also discuss an example system that use the concatenated Walsh/m-sequence scheme in a CDMA system that is suitable for variable source rate applications. In Chapter 3 , we evaluate the bit error probability performance when the concatenated Walsh $/ \mathrm{m}$-sequence is used for the forward link of a single cell CDMA system. In this chapter, we observe the effect of changing some of the parameters of the spreading code. The use of multipath diversity is discussed in Chapter 4 where a modified Rake receiver is proposed for applications in some environments. In Chapter 5 , the system performance is evaluated for a multicell application. Finally, in Chapter 6, conclusions and future research suggestions are discussed. 


\section{Chapter 2}

\section{Background}

In this chapter, we describe the parameters of some codes used for bandwidth spreading in wireless CDMA. We also discuss the concatenated orthogonal/PN spreading code and a transmitter structure when this coding scheme is applied in a multimedia system that involves variable rate information sources. Also, we highlight issues relating to the wireless channel and its modeling for our analysis. Finally, we discuss the use of diversity to improve performance in wireless CDMA systems.

\subsection{Parameters of Spreading Codes}

Channel separation is essential for reducing adjacent channel interference in a multiple access system. In CDMA systems, channel separation is determined primarily by the type of code sequences used. Also, the types of codes used set bounds on other system capabilities including synchronization, bit error rate (BER) performance and the spectral occupancy of the codes [5]. The performance measure of primary interest in this thesis is the BER. A large number of parameters can be used to describe the spreading codes in a CDMA system, but we will look primarily at those parameters that are important to BER analysis. First, we present a simple model for a binary phase shift keyed (BPSK) spread spectrum multiple access system. We then discuss the correlation parameters of spreading codes. Finally, we look at issues involved in selecting a code set that will improve the performance of a CDMA system. 


\subsubsection{System and Channel Model}

The system which may not be time synchronous has $K$ transmitters. In a time synchronous system, the signals from the different transmitters are chip and bit aligned when they arrive at the receiver. The same radio frequency (RF) band is utilized by each of the transmitters with a common center angular frequency of $\omega_{c}=2 \pi f_{c}$. In order to simplify analysis, all users are assumed to transmit with the same average power $\mathcal{P}$. Let the $k$ th user's data signal be denoted by $b_{k}(t)$, which is a sequence of unit amplitude, positive and negative rectangular pulses of duration $T$. It is assumed that the $n$th data pulse, denoted as $b_{n}^{k 1}$ takes on values +1 and -1 with equal probability. The $k$ th user is assigned a spreading code sequence $\boldsymbol{a}_{k}$ of elements in $\{+1,-1\}$. We can represent the $k$ th user's code sequence as

$$
a_{k}(t)=\sum_{j=-\infty}^{j=\infty} a_{j}^{k} \psi\left(t-j T_{c}\right)
$$

where $a_{j}^{k}$ denotes the $j$ th element of the spreading code sequence $\boldsymbol{a}_{k}$, and $\psi(t)$ is an arbitrary code pulse waveform which is time limited to a duration of $T_{c}$. The energy in a code pulse or "chip" is normalized such that $\int_{0}^{T_{c}} \psi^{2}(t) d t=T_{c}$. All the code sequences allocated to the different users have the same period $M$ so that $a_{n}^{k}=a_{M+n}^{k}$. For the moment, we assume that there are $M$ chips per bit period $T$. Both the code sequence and the data signal are used to modulate the carrier signal using BPSK to produce the signal $s_{k}(t)$ given by

$$
s_{k}(t)=\sqrt{2 \mathcal{P}} a_{k}(t) b_{k}(t) \cos \left(\omega_{c} t+\theta_{k}\right)
$$

where $\theta_{k}$ represent the phase of the carrier at the $k$ th transmitter.

\footnotetext{
${ }^{1}$ We use this notation to be consistent with other works in this area $[6,7,8]$.
} 


\subsubsection{Autocorrelation parameters}

The received signal for the case where there is only one user, i.e. $K=1$, is given by

$$
r_{1}(t)=s_{1}(t)+n(t)
$$

where $n(t)$ is the additive white Gaussian noise (AWGN) process with two-sided power spectral density $N_{0} / 2$. For a multipath channel with $L+1$ equal amplitude paths, the received signal is given by

$$
r_{1}(t)=\sum_{n=0}^{L} s_{1}\left(t-\tau_{n}\right)+n(t)
$$

where $\tau_{n}$ is the transmission delay of the signal through the $n$th path. The paths are assumed to be arranged in ascending order of transmission delays, i.e., $\tau_{0}<\tau_{1}<\cdots<\tau_{L}$. Here, we have also assumed that the channel introduces no amplitude or phase distortion, and the paths have unity gain.

If the received signal is the input to a Conventional Single-User Detector (correlation receiver) [9] that is matched to the signal from the zeroth path, the output is

$$
Z_{1}=\int_{0}^{T} r_{1}(t) a_{1}(t) \cos \left(\omega_{c} t\right) d t
$$

We assume coherent demodulation and that the receiver is capable of acquiring time synchronization with the signal from the zeroth path. By the acquisition of time synchronization, we mean that the despreading code sequence at the receiver is synchronized in time to the spreading code sequence used at the transmitter. Thus, we can set $\theta_{1}$ and $\tau_{0}$ to zero without loss of generality. Transmission delays of the signal through the other paths are measured relative to $\tau_{0}$. We also assume that $\tau_{L}<T$, which means that only two adjacent bits will be considered in the self interference. Consequently, the output of the correlation receiver becomes 


$$
\begin{aligned}
Z_{1}= & \sqrt{\frac{\mathcal{P}}{2}} T\left\{b_{0}^{1}+\sum_{n=1}^{L}\left\{\int_{0}^{\tau_{n}} b_{-1}^{1} a_{1}(t) a_{1}\left(t-\tau_{n}\right) d t+\int_{\tau_{n}}^{T} b_{0}^{1} a_{1}(t) a_{1}\left(t-\tau_{n}\right) d t\right\} \cos \left(\phi_{n}\right)\right\} \\
& +\eta \\
= & \sqrt{\frac{\mathcal{P}}{2}} T\left\{b_{0}^{1}+\sum_{n=1}^{L}\left[b_{-1}^{1} R_{1,1}\left(\tau_{n}\right)+b_{0}^{1} \hat{R}_{1,1}\left(\tau_{n}\right)\right] \cos \left(\phi_{n}\right)\right\}+\eta
\end{aligned}
$$

. In practical communication systems, $f_{c} \gg T^{-1}$; consequently, in (2.7), we have neglected the double frequency terms because of the low-pass filter after the correlation receiver [1]. The term $\phi_{n}=\omega_{c} \tau_{n}$ is the phase of the carrier signal through the $n$th path relative to the one through the zeroth path at the time of reception, while $\eta$ is the low-pass version of $n(t)$ after correlation with $a_{1}(t) \cos \left(\omega_{c} t\right)$, and is a zero mean Gaussian random variable with variance $N_{0} T / 4 . b_{0}^{1}$ is the current bit being detected, while $b_{-1}^{1}$ is the previous bit.

The integrals in (2.6) give the autocorrelation parameters of the spreading code sequence. These integrals are defined as the continuous time partial autocorrelation functions [6], and are given by

$$
\begin{aligned}
R_{1,1}\left(\tau_{n}\right) & =\int_{0}^{\tau_{n}} a_{1}(t) a_{1}\left(t-\tau_{n}\right) d t \\
& =C_{1,1}(l-M) \hat{R}_{\psi}\left(\tau_{n}^{\prime}\right)+C_{1,1}(l+1-M) R_{\psi}\left(\tau_{n}^{\prime}\right)
\end{aligned}
$$

and

$$
\begin{aligned}
\hat{R}_{1,1}\left(\tau_{n}\right) & =\int_{\tau_{n}}^{T} a_{1}(t) a_{1}\left(t-\tau_{n}\right) d t \\
& =C_{1,1}(l) \hat{R}_{\psi}\left(\tau_{n}^{\prime}\right)+C_{1,1}(l+1) R_{\psi}\left(\tau_{n}^{\prime}\right)
\end{aligned}
$$

where $l=\left\lfloor\tau_{n} / T_{c}\right\rfloor$ is the integer part of the $n$th path delay normalized to a chip duration and $\tau_{n}^{\prime}=\tau_{n}-l T_{c} . C_{1,1}(l)$ is the discrete aperiodic autocorrelation function defined as 
[10]

$$
C_{1,1}(l)=\left\{\begin{aligned}
\sum_{j=0}^{M-1-l} a_{j}^{1} a_{j+l}^{1} & , \quad 0 \leq l \leq M-1 \\
M-1+l & \\
\sum_{j=0} a_{j-l}^{1} a_{j}^{1}, & 1-M \leq l<0 \\
0, & |l|=M .
\end{aligned}\right.
$$

Refering to $(2.8)$ and $(2.9), R_{\psi}\left(\tau_{n}^{\prime}\right)$ and $\hat{R}_{\psi}\left(\tau_{n}^{\prime}\right)$ represent the partial autocorrelation function of the chip waveform defined as [6]

$$
\begin{aligned}
\hat{R}_{\psi}(s) & =\int_{s}^{T_{c}} \psi(t) \psi(t-s) d t, \\
\text { and } \quad R_{\psi}(s) & =\hat{R}_{\psi}\left(T_{c}-s\right) .
\end{aligned}
$$

The usual periodic autocorrelation function is defined as [10]

$$
\theta_{1}(l)=\sum_{j=0}^{M-1} a_{j}^{1} a_{j+l}^{1}, \quad \forall \text { integer } \quad l .
$$

There are two cases to be considered in (2.6). First of all, if $b_{-1}^{1}=b_{0}^{1}$, then the sum of the partial autocorrelation functions result in even (periodic) autocorrelation functions given by

$$
b_{0}^{1}\left[R_{1,1}\left(\tau_{n}\right)+\hat{R}_{1,1}\left(\tau_{n}\right)\right]=b_{0}^{1}\left[\theta_{1}(l) \hat{R}_{\psi}\left(\tau_{n}^{\prime}\right)+\theta_{1}(l+1) R_{\psi}\left(\tau_{n}^{\prime}\right)\right] .
$$

However, if $b_{-1}^{1} \neq b_{0}^{1}$, then the sum of the partial autocorrelation functions result in odd autocorrelation functions given by

$$
b_{0}^{1}\left[\hat{R}_{1,1}\left(\tau_{n}\right)-R_{1,1}\left(\tau_{n}\right)\right]=b_{0}^{1}\left[\hat{\theta}_{1}(l) \hat{R}_{\psi}\left(\tau_{n}^{\prime}\right)+\hat{\theta}_{1}(l+1) R_{\psi}\left(\tau_{n}^{\prime}\right)\right] .
$$

We have used the relationships that $\theta_{1}(l)=C_{1,1}(l)+C_{1,1}(l-M)$ and $\hat{\theta}_{1}(l)=C_{1,1}(l)-$ $C_{1,1}(l-M)$. The performance of the system in a multipath channel environment will thus depend on the spreading code sequence's even and odd autocorrelation parameters. Before we examine how code sequences can be optimized to improved performance in such systems, we consider the situation in which there is more than one transmitter, i.e. $K>1$. 


\subsubsection{Cross-correlation parameters}

The received signal for the case where there are $K>1$ transmitters is given by

$$
r(t)=\sum_{k=1}^{K} s_{k}\left(t-\tau_{k}\right)+n(t) .
$$

In (2.16), it is assumed that there is only one transmission path between each transmitter and the receiver and that this path does not introduce any amplitude or phase distortion. The signal from the $k$ th transmitter experiences a transmission delay of $\tau_{k}$ before reception.

If this received signal is the input to a correlation receiver that is matched to the signal from the $i$ th transmitter, the output is

$$
Z_{i}=\int_{0}^{T} r(t) a_{i}(t) \cos \left(\omega_{c} t\right) d t
$$

We assume coherent demodulation and that the receiver is capable of acquiring time synchronization with the signal from the $i$ th transmitter. Thus, we can set $\theta_{i}$ and $\tau_{i}$ to zero without loss of generality. Transmission delays of the signals from the other transmitters are measured relative to $\tau_{i}$. We also measure the phase of the carriers from the other transmitters relative to $\theta_{i}$. The output of the correlation receiver becomes

$$
\begin{aligned}
Z_{i}= & \sqrt{\frac{\mathcal{P}}{2}} T\left\{b_{0}^{i}+\sum_{\substack{k=1 \\
k \neq i}}^{K}\left\{\int_{0}^{\tau_{k}} b_{-1}^{k} a_{i}(t) a_{k}\left(t-\tau_{k}\right) d t+\int_{\tau_{k}}^{T} b_{0}^{k} a_{i}(t) a_{k}\left(t-\tau_{k}\right) d t\right\} \cos \left(\phi_{k}\right)\right\} \\
& +\eta \\
= & \sqrt{\frac{\mathcal{P}}{2}} T\left\{b_{0}^{i}+\sum_{\substack{k=1 \\
k \neq i}}^{K}\left[b_{-1}^{k} R_{k, i}\left(\tau_{k}\right)+b_{0}^{k} \hat{R}_{k, i}\left(\tau_{k}\right)\right] \cos \left(\phi_{k}\right)\right\}+\eta
\end{aligned}
$$

where $\eta$. is the low-pass version of $n(t)$ after correlation with $a_{i}(t) \cos \left(\omega_{c} t\right)$, which is a zero mean Gaussian random variable with variance $N_{0} T / 4 . b_{0}^{i}$ is the current bit of the $i$ th transmitter that is being detected. $b_{-1}^{k}$ and $b_{0}^{k}$ are the two successive bits of the $k$ th 
transmitter that overlap $b_{0}^{i} . \quad \phi_{k}=\theta_{k}-\omega_{c} \tau_{k}$ is the phase of the carrier from the $k$ th transmitter at the receiver.

The integrals in (2.18) give the cross-correlation parameters of the spreading code sequences. They are defined as the continuous time partial cross-correlation functions $[6]$, and are given by

$$
\begin{aligned}
R_{k, i}\left(\tau_{k}\right) & =\int_{0}^{\tau_{k}} a_{i}(t) a_{k}\left(t-\tau_{k}\right) d t \\
& =C_{k, i}(l-M) \hat{R}_{\psi}\left(\tau_{k}^{\prime}\right)+C_{k, i}(l+1-M) R_{\psi}\left(\tau_{k}^{\prime}\right)
\end{aligned}
$$

and

$$
\begin{aligned}
\hat{R}_{k, i}\left(\tau_{k}\right) & =\int_{\tau_{k}}^{T} a_{i}(t) a_{k}\left(t-\tau_{k}\right) d t \\
& =C_{k, i}(l) \hat{R}_{\psi}\left(\tau_{k}^{\prime}\right)+C_{k, i}(l+1) R_{\psi}\left(\tau_{k}^{\prime}\right)
\end{aligned}
$$

where $C_{k, i}(l)$ is the discrete aperiodic cross-correlation function defined as [10]

$$
C_{k, i}(l)=\left\{\begin{aligned}
\sum_{j=0}^{M-1-l} a_{j}^{i} a_{j+l}^{k}, & 0 \leq l \leq M-1 \\
\sum_{j=0}^{M-1+l} a_{j-l}^{i} a_{j}^{k}, & 1-M \leq l<0 \\
0, & |l|=M .
\end{aligned}\right.
$$

The parameters $R_{\psi}\left(\tau_{k}^{\prime}\right)$ and $\hat{R}_{\psi}\left(\dot{\tau}_{k}^{\prime}\right)$ are as defined in (2.11) and (2.12). If we measure $\tau_{k}(\bmod T), l=\left\lfloor\tau_{k} / T_{c}\right\rfloor$ is the integer part of the transmission delay of the signal from the $k$ th transmitter normalized to a chip duration, while $\tau_{k}^{\prime \prime}=\tau_{k}-l T_{c}$. The usual periodic cross-correlation function of two code sequences $\boldsymbol{a}_{i}$ and $\boldsymbol{a}_{k}$ is defined as [10]

$$
\theta_{k, i}(l)=\sum_{j=0}^{M-1} a_{j}^{i} a_{j+l}^{k}, \quad \forall \text { integer } \quad l .
$$

Following the same analysis as in the single user, multipath channel case, if $b_{0}^{k}=b_{-1}^{k}$, the output of the correlation receiver depends on the even or periodic cross-correlation functions $\theta_{k, i}(l)=C_{k, i}(l)+C_{k, i}(M-l)$ and $\theta_{k, i}(l+1)$. If $b_{0}^{k} \neq b_{-1}^{k}$, the output of the 
correlation receiver depends on the odd cross-correlation functions $\hat{\theta}_{k, i}(l)=C_{k, i}(l)-$ $C_{k, i}(M-l)$ and $\hat{\theta}_{k, i}(l+1)$.

\subsubsection{System Performance}

In the literature $[5,10]$, three different system performance parameters that have been considered in the selection of a spreading code are (i) the maximum interference at the output of the $i$ th correlation receiver due to the presence of the $k$ th signal; (ii) the signal to interference plus noise ratio at the output of the correlation receiver; and (iii) the BER for the $i$ th correlation receiver [10].

Massey and Uhran [11] showed that the maximum magnitude of the periodic and odd cross-correlation and the maximum magnitude of the periodic and odd autocorrelation are important in determining the maximum interference at the $i$ th correlation receiver due to the presence of the $K-1$ signals and the multipath signals. They also discuss methods for selecting a set of sequences for which these parameters are small [11].

If the signal phases $\phi_{k}$, transmission delays $\tau_{k}$ and the data bits $\left\{b_{-1}^{k}, b_{0}^{k}\right\}$ are treated as mutually independent random variables, Pursley [10] has shown that for rectangular chip waveform, and when the interference is treated as additional noise, the signal to noise ratio (SNR) at the output of the correlation receiver is given by

$$
\mathrm{SNR}=\left(\frac{1}{6 M^{3}} \sum_{\substack{k=1 \\ k \neq i}}^{K} r_{k, i}+\frac{N_{0}}{2 E_{b}}\right)^{-1}
$$

where $r_{k, i}$ is the interference parameter given by

$$
\begin{aligned}
r_{k, i}= & \sum_{l=0}^{M-1}\left\{C_{k, i}^{2}(l-M)+C_{k, i}(l-M) C_{k, i}(l+1-M)\right. \\
& \left.+C_{k, i}^{2}(l+1-M)+C_{k, i}^{2}(l)+C_{k, i}(l) C_{k, i}(l+1)+C_{k, i}^{2}(l+1)\right\} .
\end{aligned}
$$

and $E_{b}=\mathcal{P} T$ is the energy transmitted per data bit. The interference parameter in the 
SNR expression is further approximated in [10] to

$$
r_{k, i}=2 M^{2}+4 \sum_{l=1}^{M-1} C_{k, k}(l) C_{i, i}(l) .
$$

The above approximation reduces the number of correlations to be evaluated in order to find the average SNR. The spreading code which give the highest SNR can be selected for a particular propagation environment.

Yao [12], treated the carrier phases, transmission delays and data bits as random variables, and showed that the interference can be treated approximately as a zero mean Gaussian random variable. We will refer to this as the Gaussian approximation. Then, for a system using BPSK modulation, the BER is given by [12]

$$
P_{e}=\mathrm{Q}(\sqrt{\mathrm{SNR}})
$$

where

$$
\mathrm{Q}(x)=\frac{1}{\sqrt{2 \pi}} \int_{x}^{\infty} \exp \left(-t^{2} / 2\right) d t .
$$

He also obtained lower and upper bounds on the BER by applying moment space bounds [13]. The maximum periodic and odd cross correlations and the parameters $r_{k, i}$ must be computed to apply these bounds.

The average BER of phase coded CDMA systems has been commonly analyzed using the Gaussian approximation $[1,3,7,8,14]$. The average SNR which is evaluated over all the spreading sequences, is used to find the average BER. An often used assumption is that the BER performance of systems using long period spreading code sequencès can be approximated by those of systems using random sequences $[6,14]$. Under such conditions, for the rectangular chip waveform, the average SNR simplifies to [6]

$$
\mathrm{SNR}=\left(\frac{K-1}{3 M}+\frac{N_{0}}{2 E_{b}}\right)^{-1} .
$$


It is worth noting that the Gaussian approximation is not accurate in general, and often results in optimistic performance estimate $[1,15]$. Attempts have been made to improve the accuracy of the Gaussian approximation [16]. Unfortunately, the improved Gaussian approximations are still not accurate for all operating environments and cannot be used in some operating environments [15].

Another approach that has been used in evaluating the BER performance, is the utilization of the characteristic function of the interference. This approach, which is not analytically tractable, involves numerical integration. It has been used for deterministic spreading code sequences $[1,2,7]$ and random spreading code sequences [6]. The characteristic function approach which gives the exact BER performance has a computational requirement which grows as a function of the period, $M$, of the spreading code sequence and the number of users, $K$. The rate of growth depends on the operating environment. The BER performance of a CDMA system with m-sequences as the spreading code sequences has been obtained using the characteristic function approach [1, 7]. In this case, the spreading code sequences allocated to the users are fixed, and the BER was obtained for one user. For a CDMA system with random spreading code sequences, the BER obtained from this approach was an average over the spreading code [6].

Finally, Monte Carlo simulation techniques have been used to evaluate the BER performance [15]. The output of the correlation receiver is obtained, after generating the relevant random variables. The number of bits in error is divided by the total number of bits to give the average BER. Semi-analytical approaches have also been considered [15], where the conditional BER is averaged over the different interference values. For reasonable accuracies, the number of bits is very large when the BER is of the order of $10^{-6}$. Consequently, importance sampling techniques have been combined with Monte Carlo simulation to estimate the BER performance [15].

Whatever the approach used in evaluating the BER performance of a CDMA system, 
the selection of a good spreading code can be intuitively done by looking its correlation parameters under the desired operating environment. The maximum, mean and mean square values of the different correlation parameters of spreading codes will affect the BER performance of CDMA systems. In particular, the maximum even cross-correlation has been shown to be a very good measurement parameter for spreading code performance [17].

\subsection{Concatenated Orthogonal/ PN Spreading Code}

So far, we have seen the importance of the correlation properties of a spreading code to the performance of spread spectrum systems. A desirable objective will be to select a code that has small even and odd cross-correlations between the code sequences. The code sequences need also have impulsive even autocorrelations and small aperiodic autocorrelations. There exist families of spreading codes which satisfy some of these requirements, but a large amount of computational effort is required to obtain sub-families which satisfy all the requirements [5]. Orthogonal sequences such as Walsh sequences will be desirable as spreading code sequences in a system where transmissions can be synchronized at the chip level, but, when there is a loss of synchronization, Walsh sequences have been shown to perform very poorly compared to PN sequences [17]. On the other hand, PN sequences have ideal autocorrelation parameters [5], but because of their finite cross-correlation values, they are inferior to Walsh sequences in synchronous spread spectrum systems.

A new sequence that can be of interest in CDMA systems is the concatenation of two different sequences that have some desired properties. Here, by concatenation we mean that the element in each position of the new sequence, is the product of the elements in corresponding positions of the two sequences. The motivation for doing this is that it is 
suggested in [18] that the concatenation of a common PN sequence to orthogonal Walsh sequences tend to randomize their unsatisfactory cross-correlations at non zero time delay. Concatenated Walsh/PN sequences are used in the forward links of the CDMA system described in the IS-95 standard. The Bandwidth spreading by a concatenation of Walsh sequences to a common PN sequence has been shown to give desirable BER performance in a spread spectrum system when the Walsh codes are synchronized at the chip level [3]. In [3], concatenated Walsh/m-sequences are used to spread the bandwidth of signals transmitted from a base station (where synchronization at the chip level is practicable) to several receivers. Since the transmission delays of the signals to a particular receiver are the same, these signals will be orthogonal at that receiver. But, the presence of multipaths with different transmission delays will result in the presence of interference in the output of a correlation receiver that is matched to a particular signal. However, the average interference is small compared to that of a system where bandwidth spreading is by Walsh sequences alone. The BER performance of the forward link of a CDMA system using concatenated Walsh/m-sequences for bandwidth spreading is shown to be superior to that of a system using m-sequences alone [3]. Also, concatenated Walsh/PN codes have been used in CDMA systems involving variable rate sources (suitable for multimedia applications). Such systems, called Multi-Code CDMA [19] will be discussed in the next section.

\subsubsection{A Multi-Code CDMA System}

Multi-Code CDMA has been studied as a suitable multiple access technique that can meet the ever increasing demand for high user data rate [19]. Also, with the need for the introduction of multimedia services into wireless networks, Multi-Code CDMA has been suggested as a candidate access technique that can effectively allocate channel resources to variable rate sources $[4,19]$. 
In a Multi-Code CDMA system, signals from the information sources are divided into several signals of constant rate $R$ using serial to parallel conversion. Signals from the higher rate sources will be converted to several rate $R$ signals while signals from the lower rate sources might be converted to one or a few rate $R$ signals. When the signaling rate of a source is less than $R$, the resulting single rate $R$ signal from this source is not transmitted all the time, thereby reducing the interference it would have caused to other users. Also, when the signaling rate of a source is not an integral multiple of $R$, the number of rate $R$ signals from this source should be chosen to give a combined rate that is just higher than the source rate. These rate $R$ signals will not be active all the time.

The bandwidths of the rate $R$ signals that are derived from the same information source are spread with concatenated Walsh/PN sequences. Note that the common PN sequence used in the concatenated Walsh/PN sequence is unique to that information source. A transmitter structure for Multi-Code CDMA is presented in Figure 2.1.

Based on the block diagram of Figure 2.1, let the $j$ th rate $R$ signal derived from the $i$ th information source be denoted as $b_{i, j}(t)$. These signals are modeled as sequences of unit amplitude, positive and negative rectangular pulses of duration $T_{R}$. Thus, the $n$th pulse in the $j$ th sequence, denoted as $b_{n}^{i, j}$ has an amplitude of +1 or -1 for $n T_{R} \leq t \leq(n+1) T_{R}$. Since the source outputs bits are equally likely to be +1 or $-1, b_{n}^{i, j}$ is also equally likely to be +1 or -1 . We denote the Walsh sequence used in forming the concatenated Walsh/PN spreading code sequence for the $j$ th rate $R$ signal by $w_{j}(t)$, and the common PN. sequence that is unique to the information source $i$ is also denoted by $p_{i}(t)$. Thus, for rectangular chip waveform, the concatenated sequence is given by $a_{i, j}(t)=p_{i}(t) w_{j}(t)$. The combined output from information source $i$ after bandwidth spreading; is given by

$$
e_{i}(t)=\sum_{j=1}^{m} b_{i, j}(t) a_{i, j}(t)
$$

where $m$ is the number of rate $R$ signals that are derived from the information source $i$. 


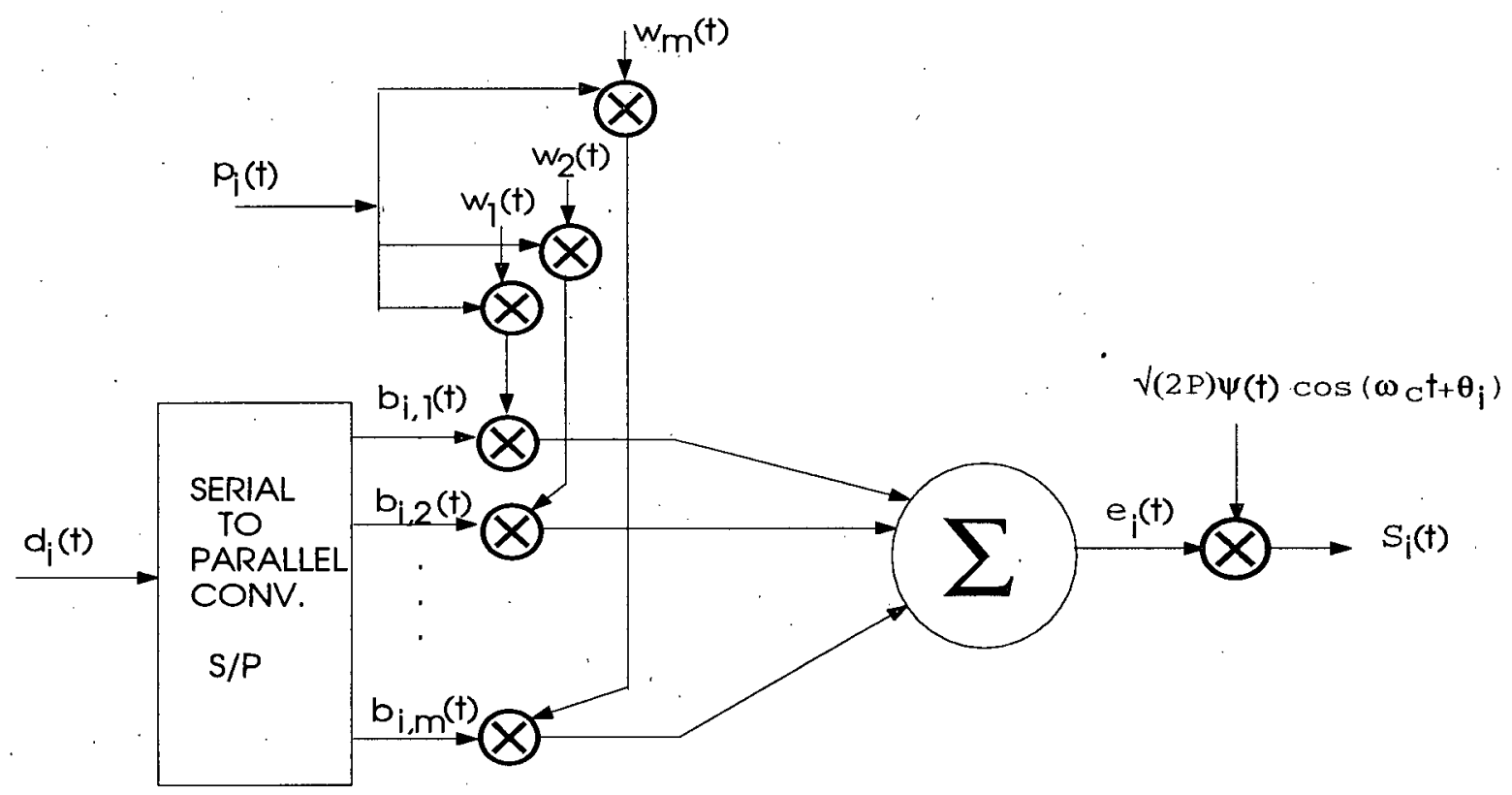

Figure 2.1: A transmitter structure for Multi-Code CDMA

The composite signal $e_{i}(t)$ is used to modulate an RF carrier using BPSK, to obtain the transmitted signal given by

$$
\begin{aligned}
s_{i}(t) & =\operatorname{Re}\left\{\sqrt{2 \mathcal{P}} \sum_{j=1}^{m} a_{i, j}(t) b_{i, j}(t) \exp \left(j \omega_{c} t+\theta_{i}\right)\right\} \\
& =\sqrt{2 \mathcal{P}} \sum_{j=1}^{m} a_{i, j}(t) b_{i, j}(t) \cos \left(\omega_{c} t+\theta_{i}\right)
\end{aligned}
$$

where $\theta_{i}$ is the phase of the carrier at the $i$ th information source, and Re denotes the real part.

The signals corresponding to the same source will be orthogonal at the receiver in applications over single path channels. Consequently, these signals will cause no mutual interference to each other. It should be noted that there will be interference caused by the signals from other sources. Also, the presence of multipath signals will result in some mutual interference among the signals corresponding to the same source. The power of 
this mutual interference signal is small in a system using the concatenated Walsh/PN spreading sequences compared to that using Walsh sequences. In the following section, we present a description of time dispersive channels with amplitude and phase distortions.

\subsection{Channel Model}

In many applications, the wireless channel is not only characterized by multipaths (caused by scattering) and a stationary AWGN process, but also by non-stationary processes known as fading. Fading is the distortion of the amplitudes of the signals transmitted through the multipaths. In general, fading of the signals through the different paths are correlated, but have often been treated in the literature as independent to simplify system analysis $[1,7]$. Thus, the wireless channel has been modeled as a Wide Sense Stationary Uncorrelated Scattering (WSSUS) process with frequency selectivity $[1,7]$. For an input signal of

$$
s(t)=\operatorname{Re}\left\{u(t) \exp \left(j \omega_{c} t\right)\right\},
$$

the output of a WSSUS channel is given by

$$
y(t)=\sum_{n=0}^{L} \boldsymbol{\operatorname { R e }}\left\{\alpha_{n}(t) u\left(t-\tau_{n}\right) \exp \left(j \omega_{c}\left(t-\tau_{n}\right)\right\}+n(t)\right.
$$

where $\alpha_{n}(t)$ is the complex amplitude gain of the $n$th path. When the rate of fading is slow compared to the bit rate, the dependence on time of the complex gain coefficients can be dropped during the interval of several bits. Thus, the fading parameters can be approximated as random variables. Consequently, for the $n$th multipath, $\alpha_{n}(t)=\alpha_{n}$ is given by [7]

$$
\begin{array}{r}
\alpha_{0}=\beta+\gamma_{0} A_{0} \exp \left(j \theta_{0}\right) \\
\text { and } \quad \alpha_{n}=\gamma_{n} A_{n} \exp \left(j \theta_{n}\right), 1 \leq n \leq L
\end{array}
$$


where $\gamma_{n} A_{n}$ denotes the amplitude gain of the signal through the $n$th path and $\theta_{n}$ accounts for the carrier phase distortion introduced by the channel on the signal through the $n$th path. In the model, $\tau_{n}$ is the transmission delay of the signal through the $n$th path. It should be noted that the zeroth path may consist of an unfaded specular signal of amplitude $\beta \neq 0$ and a faded signal. In the foregoing, $\gamma_{n}^{2}$ denotes the signal power gain in the $n$th faded path, while $A_{n}$ denotes the random variable that characterizes the fading on the signal transmitted through the $n$th path. $A_{n}$ has unity second moment. The parameters $\tau_{n}$ and $\theta_{n}$ which are also random processes have been approximated as random variables during the interval of several bits for the same reason of slow variation with respect to the duration of several bits. The distributions that have been used for the random variables $A_{n}, \theta_{n}$ and $\tau_{n}$ are discussed in the following sections.

\subsubsection{Statistics of Fading}

There is some disagreement about the statistics of the fading of signals through multipath channels in the literature [20]. One investigation reported the fading distributions to be Rayleigh or lognormal with lognormal showing a better fit, while another shows it to be Rayleigh or lognormal with Rayleigh showing a better fit. In [20], it was reported to be lognormally distributed over both local and global areas, with a log-mean value that decreases almost linearly with increasing excess delay. In [21], the fading statistics is found to be between a best fit Rayleigh distribution and a best fit Rician distribution in an outdoor environment. In indoor environment, [21] finds the fading distribution to be closer to a Rician distribution than for outdoor environment. In essence, indoor signals consist mostly of a specular component. In some applications, the fading has been modeled with very generalized distributions like the Nakagami m-distributions [22].

The multipath components are often shown to be mutually correlated. In [23], this correlation is modeled by the dependence of the fading amplitude of the $n$th path on that 
of the $(n-1)$ th path. In [20], this correlation is shown to decrease with the separation between the transmit and receive antennas and the excess delay. This correlation is also shown in [21]. However, it is common belief that the fading of signals through paths separated by more than $100 \mathrm{~ns}$ of transmission delay will no longer be correlated [24]. BER performance of CDMA systems where correlation between the fading amplitudes of multipath signals are taken into account have been shown to be insignificantly different from those of systems where this correlation is neglected [22].

\subsubsection{Statistics of Phase Distortion}

The phase distortion of the received carrier through the different paths are usually modeled as being mutually independent random variables $[1,7,25,26]$. Though the phase distortions vary with time, but by the reason of slow variation with respect to the duration of several bits, their dependence on time is dropped. The phase distortion are often approximated as mutually independent random variables that are uniformly distributed over $[0,2 \pi)[1,7,25,26]$.

\subsubsection{Statistics of Transmission Delays Through Multipaths}

The transmission delays of signals through multipaths channels are a reflection of the natural scattering structures that produce the multipaths. Because of the random size and location of these structures, the transmission delays through the different paths can be very random. In a case of wideband data collected for some environments, the transmission delays though multipath channels was found to follow a modified Poisson process [27]. In [20], this modified Poisson process was fitted to experimental data. Essentially, this modified Poisson process leads to the multipaths arriving in a clustered pattern or in a sparse pattern. The clusterness or sparseness of the multipaths depends on the environment. The transmission delays have also been modeled as being mutually independent 
random variables that are identically uniformly distributed between 0 and $\left(T-T_{c}\right)$ [7]. Two multipath signals are said to be resolvable when the time separation between the signals is greater or equal to a chip duration. In the context of spread spectrum systems, distinct paths that violate the resolvability condition are not counted separately. Instead, they are considered as a single path with a common transmission delay which is the average of the delays of the two paths. Also, the common amplitude gain and carrier phase results from the vector addition of the two signals' complex amplitude gains.

\subsection{Multipath Diversity}

The performances of communication systems are severely degraded by fading. SNR degradation can be as high as tens of decibels in Rayleigh faded channels [28]. Possible solutions to this problem are to increase transmission power, antenna size or antenna height etc. These solutions may not be practicable, and with the need for portability and reduced energy consumption, alternatives to the above solutions need to be evaluated. Such alternatives include special modulation techniques that are energy efficient yet less vulnerable to fading; special reception techniques like the use of multiple receivers etc. The use of multiple receivers and the combination of their outputs to combat fading is known as diversity.

There are several types of diversity such as space, frequency, polarization, time, and angle diversity. For digital transmission using spread-spectrum, multipath diversity which is a form of time diversity has been considered in the literature $[3,14,22]$. In wideband spread spectrum systems, where the multipath signals can be resolved by their time of arrival, different correlation receivers or "fingers" can be synchronized to the multipath signals at the chip level. The phase distortions introduced by the channel are removed and the resulting outputs of the correlation receivers are coherently combined to form a 
decision variable. The combination which can be linear or non-linear, is such that the output SNR is improved compared to the case when multipath diversity is not utilized. One such implementation is the Rake receiver where the combination gives the optimum SNR. One assumption that is made in the optimum diversity combination is that the noise and interference signals in the different fingers are uncorrelated. This is not often the case in practice, hence the Rake receiver is quasi-optimum [9]. The following combining techniques have been considered in the literature [28].

1. Selection combining.

2. Maximal-ratio combining.

3. Equal-gain combining.

We will look at the first two of these combining techniques since equal-gain combining, which is easier to implement in practice, is a special case of the maximal ratio combining technique.

\subsubsection{Selection Combining Technique}

In a selection diversity technique, the selection which can be done in the pre-detection stage gives an equivalent performance when it is done in the post-detection stage. In pre-detection selection diversity with $B$ resolvable multipath signals, the receiver selects from these $B$ signals the one with the highest SNR as input to a correlation receiver. In a post-detection selection diversity technique, $B$ different correlation receivers are synchronized at the chip levels and in phase to the $B$ resolvable multipath signals. The decision variable is the output of the receiver with the highest SNR or equivalently the one with the highest signal amplitude. We assume that the fading on the different multipath signals are slow enough such that the "search engines" in the different receivers are able 
to instantaneously estimate the SNR or signal amplitude. We also assume that the noise or interference signals on each of the fingers are independent and identically distributed.

The average SNR in a selection combining diversity technique is improved over that of the case when multipath diversity is not exploited [28].

\subsubsection{Maximal-Ratio Combining Technique}

The maximal ratio combining technique is a more general form of combining. The outputs from the $B$ fingers that have been synchronized to the signals from the $B$ paths are combined in an optimum fashion to give a decision variable. Let the output of each of the fingers at the time of sampling for a decision be denoted by $v_{n}$. A general combining law is that the outputs are combined with some weights $w_{n}$ to produce

$$
v=\sum_{n=1}^{B} w_{n} v_{n}
$$

The choice of weights determines whether the combining technique is equal gain or maximal ratio. In equal gain combining $w_{n}=1$ for all $n$, while for maximal ratio combining, the weights are chosen to optimize the output SNR. Equal gain combining, which is not an optimum technique, is easier to implement. Assuming that the path strengths are well estimated, it has been shown in [28] that the optimum combining law is

$$
v=\sum_{n=1}^{B} \alpha_{n}^{*} v_{n}
$$

where $\alpha_{n}^{*}$ is the conjugate of the complex amplitude gain of the signal transmitted through path $n$. We can see that the combination is a square law type which weight the outputs of the different fingers according to their signal strengths. Outputs from fingers which had higher amplitude signals as input are given more weight in the diversity combination than the ones from fingers with lower amplitude signals as input. The Rake receiver uses maximal ratio combination to exploit multipath diversity that may exist in spread spectrum communication systems. 


\section{Chapter 3}

\section{Performance of Concatenated CDMA codes for Forward Links}

\subsection{Introduction}

The forward link of a cellular network is the communication link through which a base station transmits signals to a mobile receiver, while the reverse link is the communication link through which it receives signals from a mobile transmitter. Since it is easier to synchronize, at the chip level, the signals to be transmitted to the different mobiles, the forward link can benefit from the advantages of using concatenated Walsh/PN sequences for bandwidth spreading in a cellular CDMA network.

In this chapter, we analyze the performance of the forward link of a cellular CDMA system when concatenated Walsh $/ \mathrm{m}$-sequences ${ }^{1}$ are used for bandwidth spreading. The performance criterion is the BER on a forward link in the presence of simultaneous transmissions to several mobile receivers. In this chapter, we assume that there is only one cell, and thus, there is no interference from other base stations. We also assume that the base station transmits at the same average power to all the mobiles. First, we present the system and channel models. We then analyze the BER performance using some of the techniques discussed in Chapter 2. Finally, we present and discuss performance results for different channel and system values.

\footnotetext{
${ }^{1}$ The PN sequences that will be used throughout this thesis are the m-sequences, and any use of the term PN sequence from now on refer to m-sequence unless otherwise stated
} 


\subsection{System Model}

There are $K$ forward links for simultaneous transmission to $K$ different mobiles. The base station allocates one Walsh sequence signal $w_{k}(t)$ for the $k$ th forward link and a common PN sequence signal $p(t)$ for concatenation with all the Walsh sequences. The Walsh sequences have the same period $M$, while the common PN sequence has a period $P$. For the ease of analysis in this thesis, we assume that $P$ and $M$ are relatively prime, i.e. they do not have any common factors. The concatenated Walsh/PN sequences will thus repeat after exactly $M P$ chips, and the starting element of each Walsh sequence will occur at every element of the PN sequence. The concatenated spreading sequence signal for the $k$ th forward link is given by $a_{k}(t)=p(t) w_{k}(t)^{2}$. Figure 3.1 shows the generation of the concatenated sequence. The $n$th element of the Walsh sequence for the $k$ th user (denoted as $w_{n}^{k}$ ) is multiplied with the $n$th element of the m-sequence (denoted as $p_{n}$ ) to produce the $n$th element of the concatenated sequence (denoted as $a_{n}^{k}$ ). Let the data signal to be transmitted through the $k$ th forward link be denoted by $b_{k}(t)$, which is a sequence of unit amplitude, positive and negative rectangular pulses of duration $T$. It is assumed that the $n$th data pulse, denoted as $b_{n}^{k}$ takes on the values +1 and -1 with equal probability. The resulting signal to be transmitted through the $k$ th forward link after bandwidth spreading is given by

$$
e_{k}(t)=b_{k}(t) a_{k}(t)
$$

This resulting signal $e_{k}(t)$ is modulated onto the phase of an RF carrier using BPSK to produce $s_{k}(t)=\sqrt{2 \mathcal{P}} e_{k}(t) \cos \left(\omega_{c} t+\theta\right)$ where $\theta$ is the carrier phase at the base station.

\footnotetext{
${ }^{2}$ We assume rectangular chip waveform throughout this thesis
} 


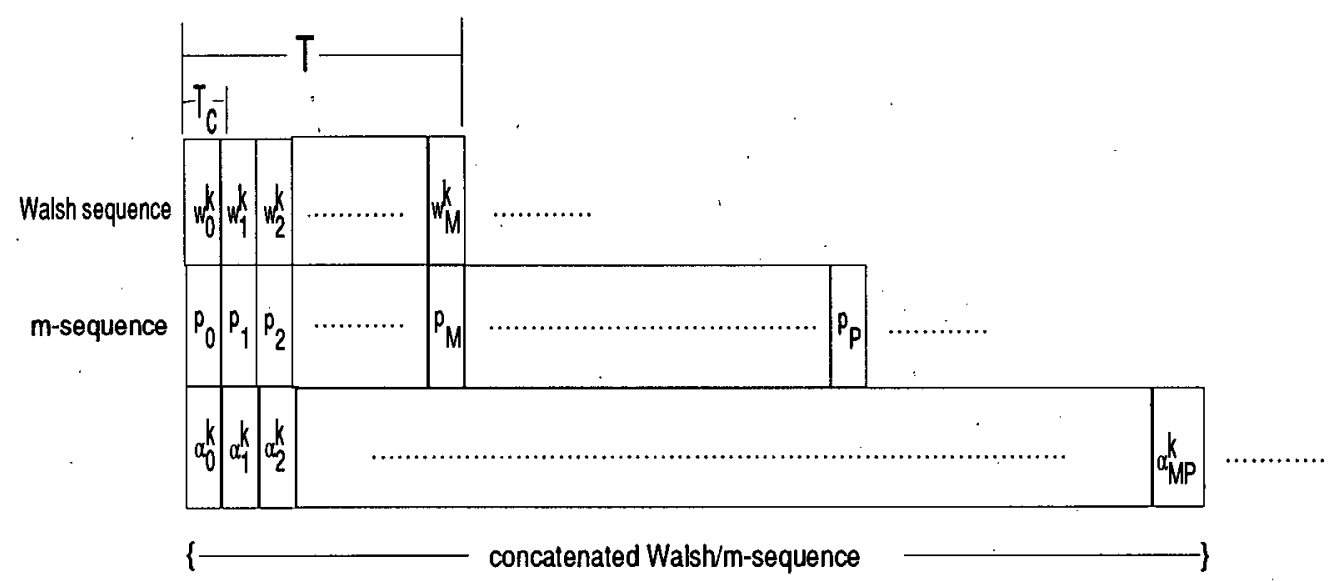

Figure 3.1: Generation of the concatenated Walsh/m-sequence

\subsection{Channel Model}

The channel is assumed to be frequency selective with $L+1$ different paths. The amplitude of the signal through each path is assumed to be Rayleigh faded, except for the zeroth path which may also contain an unfaded specular signal of amplitude gain $\beta$. When the zeroth path contains this specular unfaded signal, the zeroth path is a frequency non-selective Rician channel on its own. Thus for a total transmitted base station signal

$$
s(t)=\sum_{k=1}^{K} s_{k}(t)
$$

let

$$
y_{c}(t)=\sum_{k=1}^{K} \sqrt{2 \mathcal{P}} e_{k}(t) \exp \left(\omega_{c} t+\theta\right)
$$

be the complex representation of the total transmitted signal. The channel will produce a signal $s_{m}(t)$ at the receiver which is given by

$$
s_{m}(t)=\sum_{n=0}^{L} \boldsymbol{R e}\left\{\alpha_{n} y_{c}\left(t-\tau_{n}\right)\right\}
$$


where the complex gain coefficient $\alpha_{n}$ of the signal transmitted through the $n$th path is given by

$$
\begin{array}{r}
\alpha_{0}=\beta+\gamma_{0} A_{0} \exp \left(j \theta_{0}\right) \\
\text { and } \quad \alpha_{n}=\gamma_{n} A_{n} \exp \left(j \theta_{n}\right), 1 \leq n \leq L .
\end{array}
$$

We assume Rayleigh distributions for the variables $A_{0}, \cdots, A_{L}$. We also assume that the fading on the signals transmitted through the paths are mutually independent. While this assumption simplifies analysis, it has been found that correlation between the multipath components does not significantly affect the performance results [22]. The difference in transmission delays of the signals through the $n$th path and the zeroth path is given by $\tau_{n}=l_{n} T_{c}+\tau_{n}^{\prime}$, where $l_{n}$ is a positive integer and $\tau_{n}^{\prime}$ is assumed to be uniformly distributed between 0 and $T_{c}$. For all our analyses, we assume that the channel produce clustered multipaths. This assumption simplifies analysis and it will be shown that the BER performance obtained from this assumption is not different from that obtained from using a model of a modified Poisson process for the signal arrival times through the multipaths. Thus, we assume that the difference in transmission delays of the signals through the $n$th path and the zeroth path is given by $\tau_{n}=n T_{c}+\tau_{n}^{\prime}$. In $(3.5),\left\{\theta_{0}, \cdots, \theta_{L}\right\}$ are assumed to be uniformly distributed between 0 and $2 \pi$ and mutually independent.

The received signal at the $i$ th mobile is given by

$$
r(t)=s_{m}(t)+n(t)
$$

If this signal is the input to a correlation receiver at a mobile using the $i$ th forward link, and assuming that the despreading code at this mobile acquires synchronization with the spreading code of the signal through the zeroth path and coherent demodulation, the output of this correlation receiver is given by

$$
Z_{i}=\int_{0}^{T} r(t) a_{i}(t) \cos \left(\omega_{c} t+\Theta\right) d t
$$


For a channel where the zeroth path contains an unfaded specular signal, $\Theta=\theta$. For a channel without a specular signal in the zeroth path, $\Theta=\theta_{0}+\theta$. We now analyze the BER for a channel with no specular signal in the zeroth path. We set $\theta$ to zero without loss of generality.

\subsection{Channels with zeroth path having Rayleigh distributed fading}

The output of the correlation receiver in this case is given by

$$
Z_{i}=\sqrt{2 \mathcal{P}} \frac{T}{2}\left\{\alpha_{0} b_{0}^{i}+\eta+I_{m}+F_{m}\right\}
$$

where $\eta \sim N\left(0, \frac{N_{0}}{2 E_{b}}\right)$ is due to $n(t) . I_{m}$ is the interference due to the simultaneous transmission by the base station to other mobiles, and $F_{m}$ is interference due to the multipath signals. Both $I_{m}$ and $F_{m}$ depend on the spreading sequence allocated to the $i$ th user and the relative transmission delays of the other paths with respect to the zeroth path. We have not shown their dependence on $i$ because of the ease of simplifying notation. Since concatenated Walsh/PN spreading sequences are being used for all the forward links to the mobiles, and there is synchronization of these spreading codes at the chip level, $I_{m}=0$. We assume that the fading is slow relative to the duration of several bits so that we can find the probability of bit error conditioned on the fading variable $A_{0}$.

\subsubsection{Evaluation of BER Via the Characteristic Function Method}

The expression for the BER evaluation via the characteristic function method is given by $[7]$

$$
P_{e i}=P_{\eta}\left(A_{0}\right)+\frac{1}{\pi} \cdot \int_{0}^{\infty} \frac{\sin \left(A_{0} u\right)}{u} \Phi_{\eta}(u)\left[1-\Phi_{F_{m}}(u)\right] d u
$$


where $P_{\eta}\left(A_{0}\right)$ is the BER in the absence of multipath signals, $\Phi_{\eta}(u)$ is the characteristic function of $\eta$ and $\Phi_{F_{m}}(u)$ is the characteristic function of the multipath interference $F_{m}$. The characteristic function of $\eta$ is given by [7]

$$
\begin{array}{rr} 
& \Phi_{\eta}(u)=\exp \left(-u^{2} \sigma_{\eta}^{2} / 2\right) \\
\text { and } \quad & P_{\eta}\left(A_{0}\right)=\mathrm{Q}\left(A_{0} / \sigma_{\eta}\right) .
\end{array}
$$

The average probability of error is given by

$$
\begin{gathered}
\bar{P}_{e i}=\bar{P}_{\eta}+E_{A_{0}}\left[\frac{1}{\pi} \int_{0}^{\infty} \frac{\sin \left(A_{0} u\right)}{u} \Phi_{\eta}(u)\left[1-\Phi_{F_{m}}(u)\right] d u\right] \\
\text { where } \quad \bar{P}_{\eta}=\int_{0}^{\infty} \mathrm{Q}\left(A_{0} / \sigma_{\eta}\right) \cdot 2 A_{0} \exp \left(-A_{0}^{2}\right) d A_{0}
\end{gathered}
$$

Since $\gamma_{0}^{2}=1$ and $E\left[A_{0}^{2}\right]=1$, then we have [7]

$$
\bar{P}_{\eta}=\frac{1}{2}\left\{1-\left[1+\left(\frac{E_{b}}{N_{0}}\right)^{-1}\right]^{-1 / 2}\right\} .
$$

The parameter $F_{m}$ is independent of $A_{0}$, thus the average BER is given by [7]

$$
\begin{aligned}
\bar{P}_{e i}= & \frac{1}{2}\left\{1-\left[1+\left(\frac{E_{b}}{N_{0}}\right)^{-1}\right]^{-1 / 2}\right\} \\
& +\frac{1}{2 \sqrt{\pi}} \int_{0}^{\infty} \exp \left(-\frac{1}{4} u^{2}\right) \Phi_{\eta}(u)\left[1-\Phi_{F_{m}}(u)\right] d u .
\end{aligned}
$$

Using (3.4) and (3.7), we can express the multipath interference as

$$
\begin{aligned}
F_{m} & =\frac{2}{T} \int_{0}^{T} a_{i}(t) \sum_{n=1}^{L} \sum_{k=1}^{K} \gamma_{n} A_{n} b_{k}\left(t-\tau_{n}\right) a_{k}\left(t-\tau_{n}\right) \cos \left(\omega_{c} t+\theta_{n}-\omega_{c} \tau_{n}\right) \cos \left(\omega_{c} t+\theta_{0}\right) d t \\
& =\frac{1}{T} \int_{0}^{T} \sum_{n=1}^{L} \sum_{k=1}^{K} \gamma_{n} A_{n} b_{k}\left(t-\tau_{n}\right) a_{i}(t) a_{k}\left(t-\tau_{n}\right) \cos \left(\phi_{n}\right) d t \\
& =\frac{1}{T} \sum_{n=1}^{L} \sum_{k=1}^{K} \gamma_{n} A_{n}\left\{\int_{0}^{\tau_{n}} b_{-1}^{k} a_{i}(t) a_{k}\left(t-\tau_{n}\right) d t+\int_{\tau_{n}}^{T} b_{0}^{k} a_{i}(t) a_{k}\left(t-\tau_{n}\right) d t\right\}
\end{aligned}
$$

where $\phi_{n}=\omega_{c} \tau_{n}+\theta_{n}-\theta_{0}$ is the phase difference between the carriers in the zeroth path and the $n$th path. Using the continuous time partial cross correlation functions (2.20) 
and $(2.21)$, the multipath interference $\dot{F}_{m}$ is given by

$$
F_{m}=\frac{1}{T} \sum_{k=1}^{K} \sum_{n=1}^{L} \gamma_{n} A_{n}\left[b_{-1}^{k} R_{k, i}\left(s, \tau_{n}\right)+b_{0}^{k} \hat{R}_{k, i}\left(s, \tau_{n}\right)\right] \cos \left(\phi_{n}\right)
$$

where $s$ is the position of the starting element of the long PN sequence for the desired bit $b_{0}^{i}$. Since the period of the PN sequence and that of the Walsh sequences are relatively prime, $s$ can be modeled as a random variable that is uniformly distributed between 0 and $P-1$. For rectangular chip waveform, the characteristic function of the multipath interference is given as

$$
\Phi_{F_{m}}(u)=E\left[\exp \left(j u F_{m}\right)\right]
$$

Conditioning on the vectors $\tau=\left\{\tau_{1}, \cdots, \tau_{L}\right\}$ and $\boldsymbol{b}=\left\{b_{-1}^{1}, b_{0}^{1}, b_{-1}^{2}, b_{0}^{2}, \cdots, b_{-1}^{K}, b_{0}^{K}\right\}$ and the random variable $s$, we have

$$
\begin{aligned}
\Phi_{F_{m}}(u)= & E_{(\boldsymbol{b}, s)}\left[E _ { \boldsymbol { \tau } } \left[E _ { ( \boldsymbol { A } , \boldsymbol { \phi } ) } \left[\operatorname { e x p } \left(\frac{j u}{T} \sum_{n=1}^{L} \sum_{k=1}^{K} \gamma_{n} A_{n}\left[b_{-1}^{k} R_{k, i}\left(s, \tau_{n}\right)+b_{0}^{k} \hat{R}_{k, i}\left(s, \tau_{n}\right)\right]\right.\right.\right.\right. \\
& \left.\left.\left.\left.\cos \left(\phi_{n}\right)\right) \mid \boldsymbol{\tau}, \boldsymbol{b}, s\right]\right]\right] \\
= & E_{\left(\boldsymbol{b}_{, s}\right)}\left[\dot { E } _ { \boldsymbol { \tau } } \left[\prod _ { n = 1 } ^ { L } E _ { ( A _ { n } , \phi _ { n } ) } \left[\operatorname { e x p } \left(\frac{j u}{T} \sum_{k=1}^{K} \gamma_{n} A_{n}\left[b_{-1}^{k} R_{k, i}\left(s, \tau_{n}\right)+b_{0}^{k} \hat{R}_{k, i}\left(s, \tau_{n}\right)\right]\right.\right.\right.\right. \\
& \left.\left.\left.\left.\cos \left(\phi_{n}\right)\right) \mid \boldsymbol{\tau}, \boldsymbol{b}, s\right]\right]\right]
\end{aligned}
$$

where $\boldsymbol{A}=\left\{A_{1}, \cdots, A_{L}\right\}$ and $\boldsymbol{\phi}=\left\{\phi_{1}, \cdots, \phi_{L}\right\}$ are vectors of the fading: variables and carrier phase difference of the paths respectively. Each of $A_{n} \cos \left(\phi_{n}\right)$ are Gaussian with zero mean and variance $1 / 2$. Thus,

$$
\begin{aligned}
\Phi_{F_{m}}(u)= & E_{(\boldsymbol{b}, s)}\left[E _ { \boldsymbol { \tau } } \left[\prod _ { n = 1 } ^ { L } \operatorname { e x p } \left(\frac { - u ^ { 2 } \gamma _ { n } ^ { 2 } } { 4 T ^ { 2 } } \left(\sum _ { k = 1 } ^ { K } \left[b_{-1}^{k} R_{k, i}\left(s, \tau_{n}\right)\right.\right.\right.\right.\right. \\
& \left.\left.\left.\left.\left.+b_{0}^{k} \hat{R}_{k, i}\left(s, \tau_{n}\right)\right]\right)^{2}\right) \mid \boldsymbol{b}, s\right]\right] \\
\quad= & E_{(\boldsymbol{b}, s)}\left[\prod _ { n = 1 } ^ { L } E _ { \tau _ { n } } \left[\operatorname { e x p } \left(\frac { - u ^ { 2 } \gamma _ { n } ^ { 2 } } { 4 T ^ { 2 } } \left(\sum _ { k = 1 } ^ { K } \left[b_{-1}^{k} R_{k, i}\left(s, \tau_{n}\right)\right.\right.\right.\right.\right.
\end{aligned}
$$




$$
\left.\left.\left.\left.\left.+b_{0}^{k} \hat{R}_{k, i}\left(s, \tau_{n}\right)\right]\right)^{2}\right) \mid \boldsymbol{b}, s\right]\right] .
$$

Since $\tau_{n}=n T_{c}+\tau_{n}^{\prime}$ and $\tau_{n}^{\prime}$ is uniformly distributed between 0 and $T_{c},(3.22)$ can be expressed as

$$
\begin{aligned}
\Phi_{F_{m}}(u)= & E_{(\boldsymbol{b}, s)}\left[\prod _ { n = 1 } ^ { L } \left\{\frac { 1 } { T _ { c } } \int _ { 0 } ^ { T _ { c } } \operatorname { e x p } \left(\frac { - u ^ { 2 } \gamma _ { n } ^ { 2 } } { 4 M ^ { 2 } T _ { c } ^ { 2 } } \left(\sum _ { k = 1 } ^ { K } \left[\left(b_{-1}^{k} C_{k, i}(n-M)+b_{0}^{k} C_{k, i}(n)\right) T_{c}\right.\right.\right.\right.\right. \\
& +\left(b_{-1}^{k}\left(C_{k, i}(n+1-M)-C_{k, i}(n-M)\right)\right. \\
& \left.\left.\left.\left.\left.\left.+b_{0}^{k}\left(C_{k, i}(n+1)-C_{k, i}(n)\right)\right) \tau_{n}^{\prime}\right]\right)^{2}\right) d \tau_{n}^{\prime}\right\}\right] \\
= & E_{(\boldsymbol{b}, s)}\left[\prod_{n=1}^{L}\left\{\frac{M \sqrt{\pi}}{\gamma_{n} u C_{n}}\left\{\operatorname{erf}\left(\frac{\gamma_{n} u}{2 M}\left(B_{n}+C_{n}\right)\right)-\operatorname{erf}\left(\frac{\gamma_{n} u}{2 M} B_{n}\right)\right\}\right\}\right]
\end{aligned}
$$

where $C_{n}$ is given by

$$
\begin{array}{r}
C_{n}=\sum_{k=1}^{K}\left\{b_{-1}^{k}\left(C_{k, i}(n+1-M)-C_{k, i}(n-M)\right)+b_{0}^{k}\left(C_{k, i}(n+1)-C_{k, i}(n)\right)\right\} \\
\text { and } B_{n}=\sum_{k=1}^{K}\left[b_{-1}^{k} C_{k, i}(n-M)+b_{0}^{k} C_{k, i}(n)\right] .
\end{array}
$$

We cannot express $\Phi_{F_{m}}(u)$ in a closed form. We note that $\boldsymbol{b}$ has $2 K$ components and that there are $2^{2 K}$ different vectors with each occuring with probability of $1 / 2^{2 K}$. The characteristic function $\Phi_{F_{m}}(u)$ can be obtained numerically using the distribution of $s$ and $\boldsymbol{b}$. Consequently, the average BER can be obtained from (3.15). The computation time using this method grows linearly with $P \times K \times 2^{2 K}$.

\subsubsection{Evaluation of Bit Error Probability Via the Gaussian Approximation of the Multipath Interference}

Starting from (3.8), and since we approximate the multipath interference $F_{m}$ as having a Gaussian distribution, the sum $F_{m}+\eta$ will be a zero mean Gaussian random variable. The conditional variance of the output of the correlation receiver is given by

$$
\operatorname{var}\left(Z_{i} \mid A_{0}\right)=\operatorname{var}(\eta)+\operatorname{var}\left(F_{m}\right)
$$


From (3.17), we can express the variance of the multipath interference as

$$
\begin{aligned}
\operatorname{var}\left(F_{m}\right)= & E\left[\left(\frac{1}{T} \sum_{k=1}^{K} \sum_{n=1}^{L} \gamma_{n} A_{n}\left[b_{-1}^{k} R_{k, i}\left(s, \tau_{n}\right)+b_{0}^{k} \hat{R}_{k, i}\left(s, \tau_{n}\right)\right] \cos \left(\phi_{n}\right)\right)^{2}\right] \\
= & \frac{1}{T^{2}} E_{s}\left[E _ { \boldsymbol { \tau } } \left[E _ { ( \boldsymbol { b } , \boldsymbol { \phi } , \boldsymbol { A } ) } \left[\left(\sum _ { k = 1 } ^ { K } \sum _ { n = 1 } ^ { L } \gamma _ { n } A _ { n } \left[b_{-1}^{k} R_{k, i}\left(s, \tau_{n}\right)\right.\right.\right.\right.\right. \\
& \left.\left.\left.\left.\left.+b_{0}^{k} \hat{R}_{k, i}\left(s, \tau_{n}\right)\right] \cos \left(\phi_{n}\right)\right)^{2} \mid \boldsymbol{A}, \boldsymbol{\phi}, \boldsymbol{b}, \boldsymbol{\tau}, \boldsymbol{s}\right]\right]\right]
\end{aligned}
$$

Since $\left\{A_{n} \cos \left(\phi_{n}\right) ; n=1, \cdots, L\right\}$ are mutually independent zero mean Gaussian random variables with variances $1 / 2$, we have

$$
\begin{aligned}
\operatorname{var}\left(F_{m}\right)= & \frac{1}{T^{2}} E_{s}\left[E _ { \boldsymbol { \tau } } \left[E _ { \boldsymbol { b } } \left[\left(\sum _ { n = 1 } ^ { L } \frac { \gamma _ { n } ^ { 2 } } { 2 } \left(\sum _ { k = 1 } ^ { K } \left[b_{-1}^{k} R_{k, i}\left(s, \tau_{n}\right)\right.\right.\right.\right.\right.\right. \\
& \left.\left.\left.\left.\left.\left.+b_{0}^{k} \hat{R}_{k, i}\left(s, \tau_{n}\right)\right]\right)^{2}\right) \mid \boldsymbol{\tau}, s\right]\right]\right] .
\end{aligned}
$$

Also, since $E\left[b_{m}^{i} b_{n}^{j}\right]=0$ for $i \neq j, \forall m$ and $n$, and $E\left[b_{-1}^{k} b_{0}^{k}\right]=0$, we simplify the above, after some manipulations, to obtain

$$
\operatorname{var}\left(F_{m}\right)=\frac{1}{2 T^{2}} E_{s}\left[E_{\tau}\left[\sum_{n=1}^{L} \gamma_{n}^{2} \sum_{k=1}^{K}\left[R_{k, i}^{2}\left(s, \tau_{n}\right)+\hat{R}_{k, i}^{2}\left(s, \tau_{n}\right)\right] \mid s\right]\right]
$$

(3.31) can be simplified for the concatenated Walsh/m-sequences when $P$ is large relative to $M$. Thus we have the variance of the multipath interference as $[3,4]$

$$
\operatorname{var}\left(F_{m}\right) \cong \frac{K}{3 M} \sum_{n=1}^{L} \gamma_{n}^{2}
$$

An interesting point to note is that when $P$ is large, $\operatorname{var}\left(F_{m}\right)$ is the same as when the spreading sequence is random. It should be noted that for $P \gg M$, the variance of the multipath interference is independent of the number of integer chip durations in $\tau_{n}$. Using the Gaussian Approximation, the conditional SNR at the output of the correlation receiver is given by

$$
\operatorname{SNR}\left(A_{0}\right)=\frac{\gamma_{0}^{2} A_{0}^{2}}{\frac{N_{0}}{2 E b}+\frac{K}{3 M} \sum_{n=1}^{L} \gamma_{n}^{2}}
$$


The conditional bit error probability is consequently given by $\mathrm{Q}\left(\sqrt{\operatorname{SNR}\left(A_{0}\right)}\right)$. The average bit error probability can be found by averaging over the Rayleigh random variable $A_{0}$. This can be shown to be [1]

$$
\bar{P}_{e i}=\frac{1}{2}\left\{1-\left[1+2\left(\mathrm{SNR}^{\prime}\right)^{-1}\right]^{-1 / 2}\right\}
$$

where

$$
\mathrm{SNR}^{\prime}=\frac{\gamma_{0}^{2}}{\frac{N_{0}}{2 E_{b}}+\frac{K}{3 M} \sum_{n=1}^{L} \gamma_{n}^{2}}
$$

\subsubsection{Evaluation of Bit Error Probability Via Monte Carlo Simulation}

We can also evaluate the BER via Monte Carlo simulation.. Using this method, we randomly pick a starting element position, $s$, of the $\mathrm{PN}$ sequence to be used for the bit $b_{-1}^{i}$ from the $P$ possible element positions which are equally likely, and generate $2 M$. elements of the PN sequence following and including this starting element. We concatenate these $2 M$ elements with two periods of the Walsh sequence allocated to each of the users to obtain a $2 M$ segment of the concatenated Walsh/PN sequence for each user $k$. This segment of concatenated sequence $\boldsymbol{a}_{k}$ is used to spread the bandwidth of two adjacent data pulses of $b_{k}(t)$ with amplitudes $b_{-1}^{k}$ and $b_{0}^{k}$ which are independent and identically distributed ( iid) at +1 and -1 with equal probabilities. At the receiver, the fractional transmission delays $\left\{\tau_{n}^{\prime} ; n=1, \cdots, L\right\}$ are generated. These variables $\left\{\tau_{n}^{\prime} ; n=1, \cdots, L\right\}$ are uniformly distributed between 0 and $T_{c}$ and are iid. We also generate the path amplitude gains $\left\{\gamma_{n} A_{n} ; n=0, \cdots, L\right\}$ which are iid Rayleigh random variables. The random Gaussian noise variable $\eta$ is also generated. We obtain the multipath interference and then form the output of the correlation receiver $Z_{i}$ for the desired user. From $Z_{i}$, we check whether the desired bit is received correctly or in error. This process is repeated to receive an adequate number of error bits in order obtain a $95 \%$ confidence interval of $\pm 5 \%$ of the average simulated BER. 


\subsection{Channels with zeroth path having Rician distributed fading}

In this case, we assume that $\Theta=0$. If there is a faded signal in the zeroth path, it will add interference because of the large autocorrelation at zero time delay of the spreading sequence allocated to the desired user. We denote this interference as $I_{m}$. Thus

$$
Z_{i}=\sqrt{2 \mathcal{P}} \frac{T}{2}\left\{\beta b_{0}^{i}+\eta+I_{m}+F_{m}\right\}
$$

Let $\beta^{2}$ denote the power gain of the non-faded signal in the zeroth path, and $\gamma_{0}^{2}$ denote the power gain of the faded signal in the zeroth path. Following the same procedure as for channels with Rayleigh faded zeroth path, we can find the probability of bit error, but, in this case,

$$
\begin{aligned}
I_{m} & =\frac{2}{T} \int_{0}^{T} \gamma_{0} A_{0} b_{0}^{i} a_{i}^{2}(t) \cos \left(\omega_{c} t\right) \cos \left(\omega_{c} t+\theta_{0}\right) d t \\
& =b_{0}^{i} A_{0} \gamma_{0} \cos \left(\theta_{0}\right)
\end{aligned}
$$

\subsubsection{Evaluation of Bit Error Probability Via the Characteristic Function Method}

Here; the average BER is obtained by modifying (3.12), and is given by

$$
\begin{array}{r}
\bar{P}_{e i}=\tilde{P}_{\eta}+\left[\frac{1}{\pi} \int_{0}^{\infty} \frac{\sin (\beta u)}{u} \Phi_{\eta}(u)\left[1-\Phi_{T_{m}}(u)\right] d u\right] \\
\text { where } \quad \bar{P}_{\eta}=\mathrm{Q}\left(\beta / \sigma_{\eta}\right) .
\end{array}
$$

Here, $T_{m}=I_{m}+F_{m}$ is the total interference while $\Phi_{T_{m}}(u)$ is its characteristic function which we need to evaluate. The multipath interference $F_{m}$ is the same as before. Let $\boldsymbol{A}=\left\{A_{0}, \cdots, A_{L}\right\}$ and $\boldsymbol{\phi}=\left\{\phi_{0}, \cdots, \phi_{L}\right\}$ where $\phi_{0}=\theta_{0}$. Conditioning on the vectors $\boldsymbol{\tau}$ and $\boldsymbol{b}$, the characteristic function of the total interference is given by

$$
\Phi_{T_{m}}(u)=E_{(\boldsymbol{b}, s)}\left[E _ { \boldsymbol { \tau } } \left[E _ { ( \boldsymbol { A } , \boldsymbol { \phi } ) } \left[\operatorname { e x p } \left(\frac{j u}{T} \sum_{n=1}^{L} \sum_{k=1}^{K} \gamma_{n} A_{n}\left[b_{-1}^{k} R_{k, i}\left(s, \tau_{n}\right)+b_{0}^{k} \hat{R}_{k, i}\left(s, \tau_{n}\right)\right]\right.\right.\right.\right.
$$




$$
\begin{aligned}
& \left.\left.\left.\left.\cos \left(\phi_{n}\right)+\frac{j u}{T} b_{0}^{i} A_{0} \gamma_{0} \cos \left(\phi_{0}\right)\right) \mid \boldsymbol{\tau}, \boldsymbol{b}, s\right]\right]\right] \\
= & E_{(\boldsymbol{b}, s)}\left[E _ { \boldsymbol { \tau } } \left[\prod _ { n = 1 } ^ { L } E _ { ( A _ { n } , \phi _ { n } ) } \left[\operatorname { e x p } \left(\frac{j u}{T} \sum_{k=1}^{K} \gamma_{n} A_{n}\left[b_{-1}^{k} R_{k, i}\left(s, \tau_{n}\right)+b_{0}^{k} \hat{R}_{k, i}\left(s, \tau_{n}\right)\right]\right.\right.\right.\right. \\
& \left.\left.\left.\left.\cos \left(\phi_{n}\right)\right) \mid \boldsymbol{\tau}, \boldsymbol{b}, s\right] E_{\left(A_{0}, \phi_{0}, b_{0}^{i}\right)}\left[\exp \left(\frac{j u}{T} b_{0}^{i} A_{0} \gamma_{0} \cos \left(\phi_{0}\right)\right)\right]\right]\right]
\end{aligned}
$$

Again, each of $A_{n} \cos \left(\phi_{n}\right)$ are Gaussian with zero mean and variance 1/2. Thus,

$$
\begin{aligned}
\Phi_{T_{m}}(u)= & E_{\boldsymbol{b}, s}\left[E _ { ( \boldsymbol { \tau } ) } \left[\prod _ { n = 1 } ^ { L } \operatorname { e x p } \left(\frac { - u ^ { 2 } \gamma _ { n } ^ { 2 } } { 4 T ^ { 2 } } \left(\sum _ { k = 1 } ^ { K } \left[b_{-1}^{k} R_{k, i}\left(s, \tau_{n}\right)\right.\right.\right.\right.\right. \\
& \left.\left.\left.\left.\left.+b_{0}^{k} \hat{R}_{k, i}\left(s, \tau_{n}\right)\right]\right)^{2}\right) \mid \boldsymbol{b}, s\right]\right] \exp \left(\frac{-u^{2} \gamma_{0}^{2}}{4}\right) \\
= & E_{(\boldsymbol{b}, s)}\left[\prod _ { n = 1 } ^ { L } E _ { \tau _ { n } } \left[\operatorname { e x p } \left(\frac { - u ^ { 2 } \gamma _ { n } ^ { 2 } } { 4 T ^ { 2 } } \left(\sum _ { k = 1 } ^ { K } \left[b_{\tau 1}^{k} R_{k, i}\left(s, \tau_{n}\right)\right.\right.\right.\right.\right. \\
& \left.\left.\left.\left.\left.+b_{0}^{k} \hat{R}_{k, i}\left(s, \tau_{n}\right)\right]\right)^{2}\right) \mid \boldsymbol{b}, s\right]\right] \exp \left(\frac{-u^{2} \gamma_{0}^{2}}{4}\right) .
\end{aligned}
$$

Following the same steps as before, (3.43) can be expressed as

$$
\Phi_{F_{m}}(u)=E\left[\prod_{n=1}^{L}\left\{\frac{M \sqrt{\pi}}{\gamma_{n} u C_{n}}\left\{\operatorname{erf}\left(\frac{\gamma_{n} u}{2 M}\left(B_{n}+C_{n}\right)\right)-\operatorname{erf}\left(\frac{\gamma_{n} u}{2 M} B_{n}\right)\right\}\right\}\right] \exp \left(\frac{-u^{2} \gamma_{0}^{2}}{4}\right)(3
$$

where $B_{n}$ and $C_{n}$ are given in (3.26) and (3.25) respectively.

We cannot express $\Phi_{T_{m}}(u)$ in a closed form. However, the characteristic function can be evaluated numerically for each starting element position, $s$. This can be averaged over all the starting element positions to obtain the average BER, and the computational time is as discussed in Section 3.4.1.

\subsubsection{Evaluation of Bit Error Probability Via the Gaussian Approximation of the Multiple Access Interference}

Starting from (3.36), and since we assume that the total interference $T_{m}$ has an approximately Gaussian distribution, the sum of the total interference and the AWGN will be 
a zero mean Gaussian random variable. The conditional variance of the output of the correlation receiver is given by

$$
\operatorname{var}\left(Z_{i} \mid b_{0}^{i}=1\right)=\operatorname{var}(\eta)+\operatorname{var}\left(T_{m}\right)
$$

where $\operatorname{var}\left(T_{m}\right)$ can be shown to be equal to the sum of the variance of $I_{m}$ and $F_{m}$. Now, $\operatorname{var}\left(I_{m}\right)=\gamma_{0}^{2} / 2$, while the variance of $F_{m}$ has been derived as in (3.32). Thus, the signal to noise ratio at the output of the correlation receiver becomes

$$
\mathrm{SNR}=\frac{\beta^{2}}{\frac{N_{0}}{2 E_{b}}+\frac{\gamma_{0}^{2}}{2}+\frac{K}{3 M} \sum_{n=1}^{L} \gamma_{n}^{2}} .
$$

The average bit error rate is given by $\mathrm{Q}(\sqrt{\mathrm{SNR}})$.

\subsubsection{Evaluation of Bit Error Probability Via Monte Carlo Simulation}

The procedure for the evaluation of the average bit error probability via Monte Carlo simulation is similar to that discussed in Section 3.4.3. The only difference is that there is also a specular signal transmitted through the zeroth path. This specular signal with a deterministic amplitude of $\beta$ is not resolved in time from the faded signal. We assume that the correlation receiver is synchronized at the chip level and in phase with the specular signal.

\subsection{Numerical Results}

To present numerical results of the BER performance of the forward link of a CDMA system using the concatenated Walsh/PN spreading sequence, we utilize some actual channel parameters. We base our multipath power profile (sometimes called Multipath Intensity Profile - MIP) on the investigation done in [21]. That investigation which was done in Ottawa, considered both wide band and narrow band CDMA. The wide 
band CDMA system that was considered was a $20 \mathrm{MHz}$ system, while the narrow band system was a $1.25 \mathrm{MHz}$ system. In outdoor environments, the results of that investigation showed that more than $80 \%$ of the total received power are in the three strongest paths for both narrow and wide band systems. For a narrow band system of $1.25 \mathrm{MHz}$, the path strengths of the three strongest paths normalized to the strongest path are $0 \mathrm{~dB}$, $-15 \mathrm{~dB}$ and $-17 \mathrm{~dB}$. For a wide band system of $20 \mathrm{MHz}$, these values are $0 \mathrm{~dB},-2 \mathrm{~dB}$ and $-3 \mathrm{~dB}$. In indoor environments which consist mostly of a specular signal with very little fading, only $10 \mathrm{MHz}$ and $20 \mathrm{MHz}$ bandwidth systems were investigated. In these cases, more than $90 \%$ of the total received power are in the three strongest paths. For a $10 \mathrm{MHz}$ system, the path strengths of the three strongest paths normalized to the strongest path are $0 \mathrm{~dB},-12 \mathrm{~dB}$ and $-14 \mathrm{~dB}$, while for a $20 \mathrm{MHz}$ system, these values are $0 \mathrm{~dB},-9.5 \mathrm{~dB}$ and $-12 \mathrm{~dB}$. We can see from this investigation that the signals in the paths other than the strongest path are very weak. In order to be able to observe the effect of strong multipaths in a Rician fading environment, we utilize an arbitrary MIP of $0 \mathrm{~dB},-2 \mathrm{~dB}$ and $-3 \mathrm{~dB}$. In all of our results, we assume there are 3 paths, and that the transmission delay between the signals transmitted through two adjacent paths is between $T_{c}$ and $2 T_{c}$.

In the numerical results presented, the processing gain is assumed to be 64 . The common $\mathrm{m}$-sequences that is used in concatenating with the Walsh sequences of period 64 are as follows. For $P=2047$, the $\mathrm{m}$-sequence is generated with a shift register with a characteristic polynomial of 5001 in octal notation [29], while for $P=63,31$ and 15 the characteristic polynomials are 103, 45 and 23 respectively. For a fixed m-sequence period of $P$, the performance of the systems was found to be independent of the particular m-sequence used.

Figures 3.2, 3.3, 3.4 and 3.5 show the BER performance for the forward link of a CDMA system using the concatenated Walsh/PN spreading sequences in outdoor channel environments that are characterized by Rayleigh fading on the signal transmitted through 
the zeroth path. Different methods are used to evaluate the BER. In all four figures, the transmitted bit energy to noise density ratio $E_{b} / N_{0}$ is $30 \mathrm{~dB}$.

In Figure 3.2, results for a system operating in a channel environment with weak multipath signals show that a smaller period m-sequence provides a lower BER. This can also be observed in Figure 3.3 for a system operating in a channel environment with strong multipath signals. The difference in BER performance for different period $\mathrm{m}$-sequence averaged over all Walsh sequences is less significant, and decreases as the number of users increases. It should noted that the performance results shown in Figures 3.2 and 3.3 using the characteristic function approach are for a system where the Walsh sequence used for concatenation to form the spreading sequence for the desired user is the first one in the set. This Walsh sequence is denoted as $\operatorname{WAL}(0, T)[30]$, while the Walsh sequences used for concatenation to form the spreading sequences for the interfering users are $\operatorname{WAL}(1, T)$ to $\operatorname{WAL}(4, T)$ for systems having 2 to 5 users. The results obtained using the characteristic function approach were confirmed to closely agree with simulations using the same set of Walsh sequences.

In Figures 3.4 and 3.5, simulated average BER performance results are obtained when any one of the 64 Walsh sequences can be selected to form the spreading sequence for the desired user. The Walsh sequences used to form the spreading sequences for the interfering users are selected at random from the remaining 63 Walsh sequences which are equally likely to be selected. We could not present results evaluated by the characteristic function approach because of the large amount of time required for computation when the number of users exceeds 5 .

In Figure 3.4, results are given for a system operating in a channel with weak multipaths. We can observe that the Gaussian approximation approach for evaluating the BER is accurate. Moreover, the average difference in performance between a system with $P=2047$ and a system with $P=15$ is not significant when the channel is made up of 
weak multipaths.

In Figure 3.5, results are presented.for a system operating in a channel with strong multipath signals. The Gaussian approximation gives a somewhat pessimistic estimate of the BER. Also, it can be observed that for small values of $K$, performance gain obtained with smaller period $\mathrm{m}$-sequence is somewhat more significant when the multipath signals are strong.

Figures 3.6, 3.7, 3.8 and 3.10 show the BER for indoor channel environments that are characterized by Rician fading on the signal transmitted through the zeroth path. We assume that there is no faded signal in the zeroth path in all cases i.e. $\gamma_{0}=0$. Again, different methods are used to evaluate the BER for different periods of $\mathrm{m}$-sequence.

In Figure 3.6, results for a system operating in a channel environment with weak multipath signals are presented. The parameter $E_{b} / N_{0}$ is $10 \mathrm{~dB}$ in this case. This lower $E_{b} / N_{0}$ was used because with weak multipath signals, the BER is very low for $E_{b} / N_{0}=$ $30 \mathrm{~dB}$ and we will have numerical instability with the characteristic function approach in evaluating the integral in (3.38). On the other hand, simulating a very low BER can take a lot of computer time. Figure 3.7 also show the average BER in a channel with strong multipath signals. Because of the presence of larger strength multipath signals, an $E_{b} / N_{0}$ value of $20 \mathrm{~dB}$ is used. Both Figures 3.6 and 3.7 show the same trend that smaller period m-sequences provide lower BER than larger period m-sequences. It should again be noted that the performance results shown are for systems where the Walsh sequence used in forming the spreading sequence for the desired user is $\operatorname{WAL}(0, T)$, while $\operatorname{WAL}(1, T)$ to WAL $(4, T)$ are used in forming the spreading sequences for the interfering users.

In Figures 3.8 and 3.10 BER results are shown averaged over all the different Walsh sequences. In these cases, $E_{b} / N_{0}=30 \mathrm{~dB}$. Figure 3.8 presents results for a channel with weak multipath signals and the number of users, $K$, ranging from 45 to 64 , while Figure 3.10 presents results for systems with strong multipath signals and the number of users, 
$K$, ranging from 10 to 64 . We can observe that the Gaussian approximation is very optimistic in estimating the BER, and that there is not much difference between systems with $P=2047$ and $P=15$ in both Figures 3.8 and 3.10 .

Figure 3.9 show BER performance when the zeroth path contained a $10 \%$ faded signal, i.e. $\gamma_{0}^{2}=0.1$ and $\beta^{2}=0.9$. The Gaussian approximation is found to be closer in agreement than for a case where there is no faded signal in the zeroth path as shown in Figure 3.8. We can also observe that this $10 \%$ power faded signal results in a BER that is at least 2.5 times that for a channel without a fading signal in the zeroth path.

In Figure 3.11, we have simulated the probability density function (pdf) of the multipath interference. Since the Gaussian Approximation assumes that this interference is conditionally Gaussian, we have fixed the fading variables at $A_{1}=A_{2}=1$. For a channel with weak multipath signals $(0 \mathrm{~dB},-9.5 \mathrm{~dB},-12 \mathrm{~dB})$, the variance of the multipath interference is obtained from (3.32) as 0.05843 . The value obtained from simulation is 0.0586 . A Gaussian pdf is fitted using the mean and variance obtained from simulations. It can be observed that despite the fact that both the simulated pdf and the Gaussian fit closely agree in most of the region on a linear scale, there is a considerable difference between the areas under the tails of the pdfs under a logarithmic scale. This difference accounts for the difference between the BER obtained from simulation and that obtained from using the Gaussian Approximation. The Gaussian Approximation is based on the application of the Central Limit Theorem to the sum of the interference contributed by the signals simultaneously transmitted to the different users. The Central Limit Theorem assumes independence between the random variables that are being added, however, in the case of the interference contributed by the signals being transmitted to the different users, there are mutual correlations amongst the variables because of the common transmission delays, amplitude gains and phases of the carriers. This lack of independence means that the Central Limit Theorem can no longer be applied. 
Figure 3.12 shows simulation results for a channel with weak multipath signals and the number of users, $K$, ranging from 45 to 64 . However, we assume a value of $E_{b} / N_{0}$ of 10dB. The Gaussian Approximation becomes more accurate in this case. The explanation for this is that the system becomes more noise limited than interference limited. 


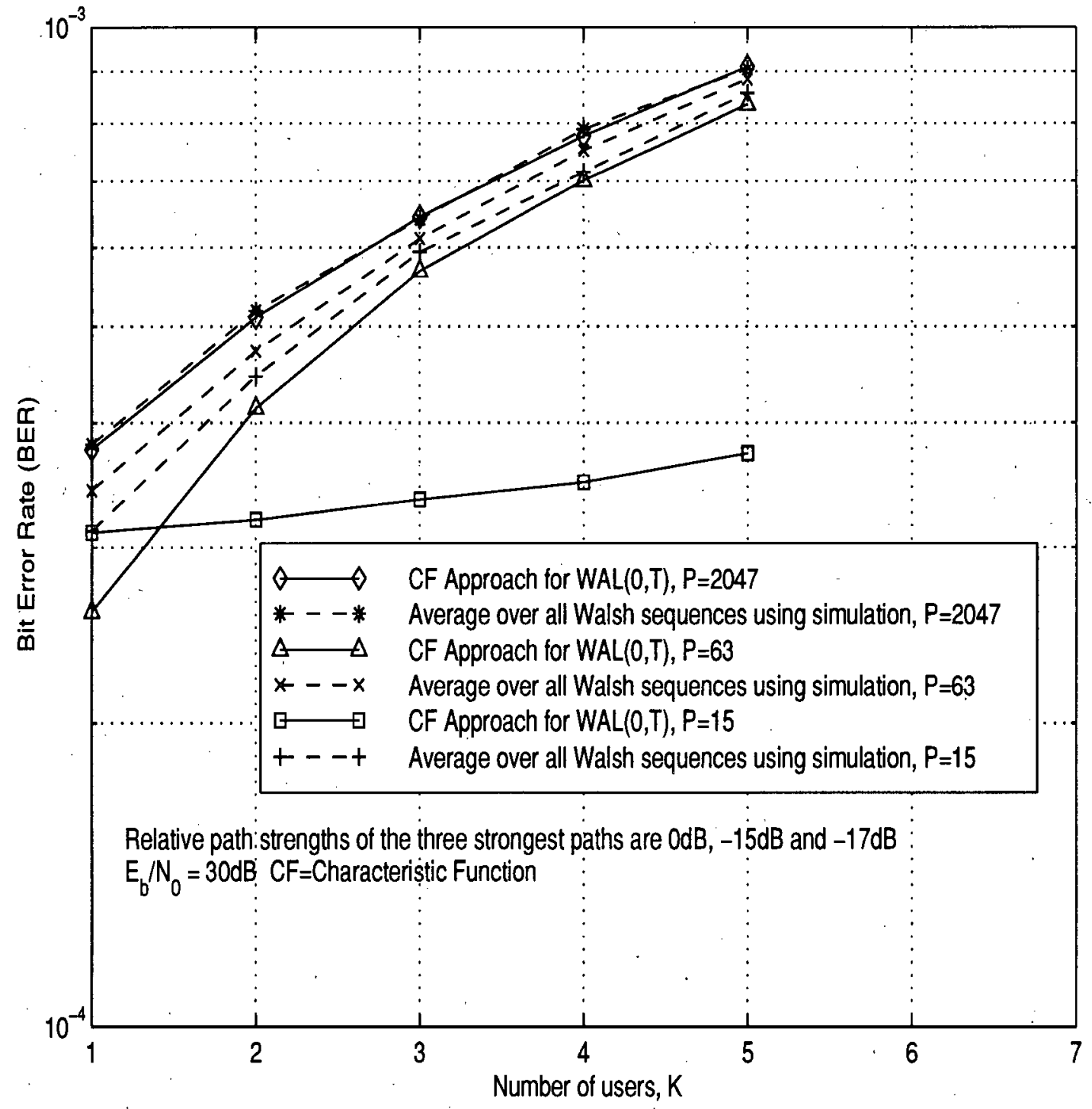

Figure 3.2: BER in a Rayleigh faded channel with weak multipaths, $M=64$ 


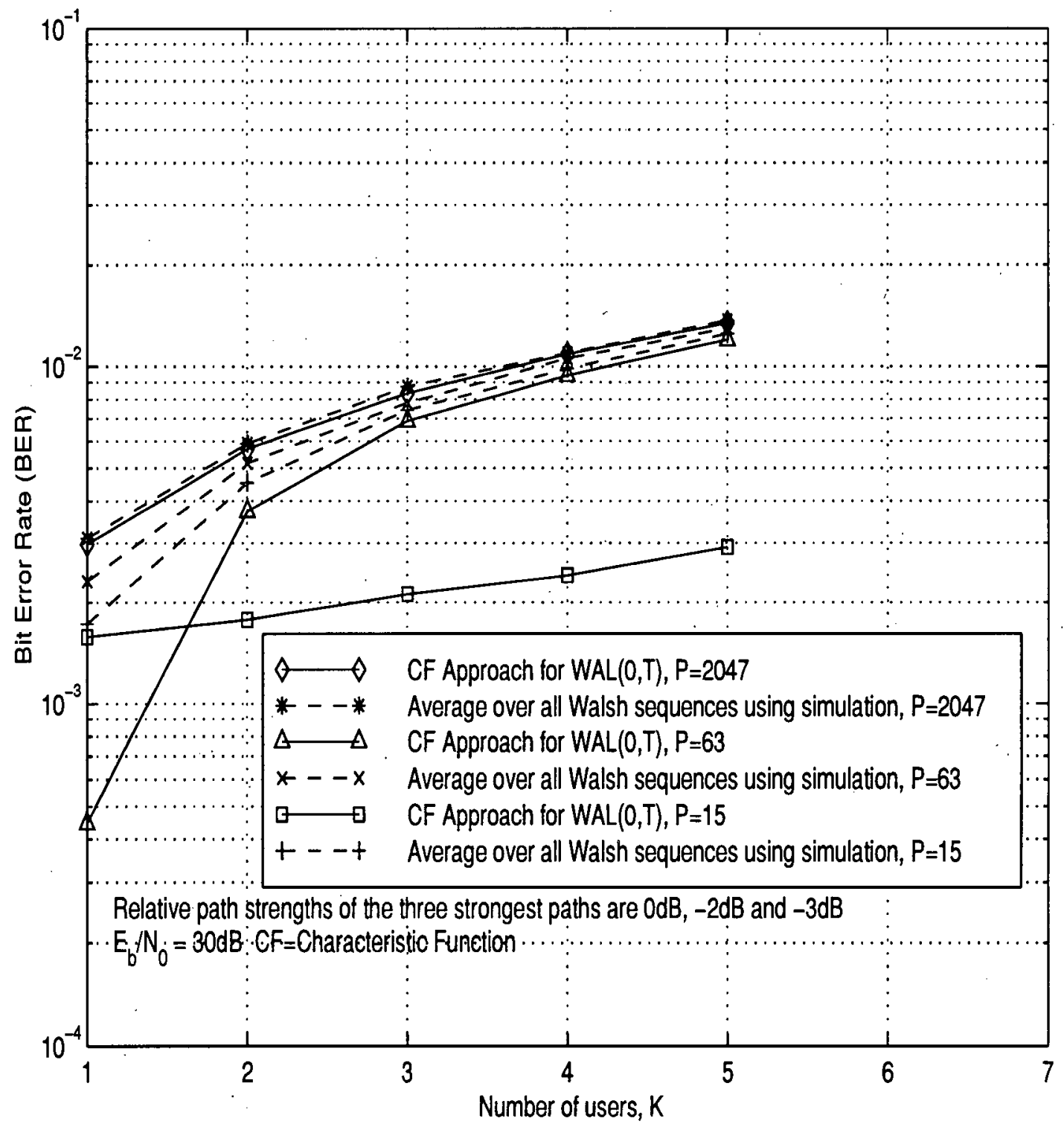

Figure 3.3: BER in a Rayleigh faded channel with strong multipaths, $M=64$ 


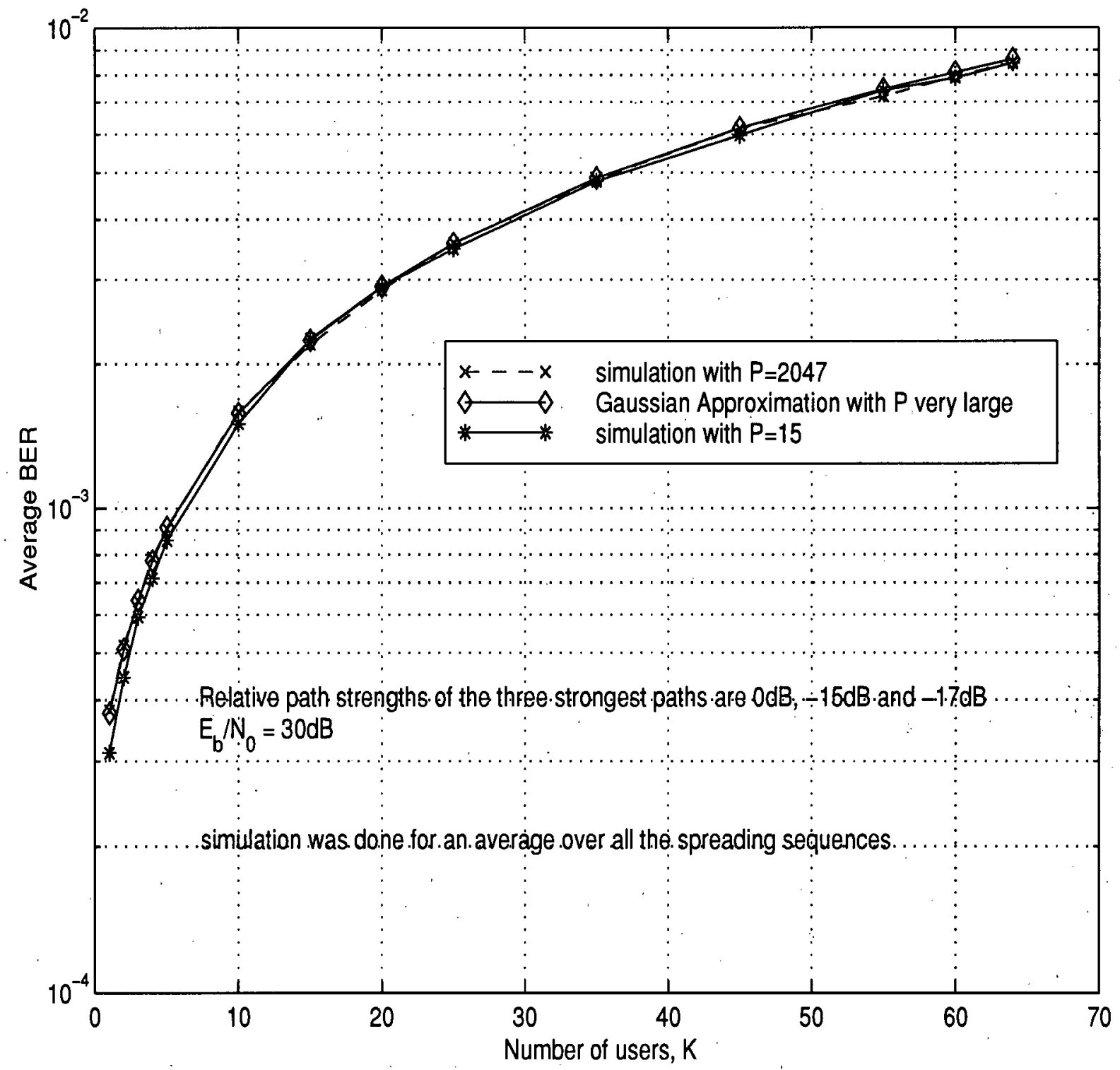

Figure 3.4: BER in a Rayleigh faded channel (with weak paths) using different evaluation techniques, $M=64$ 


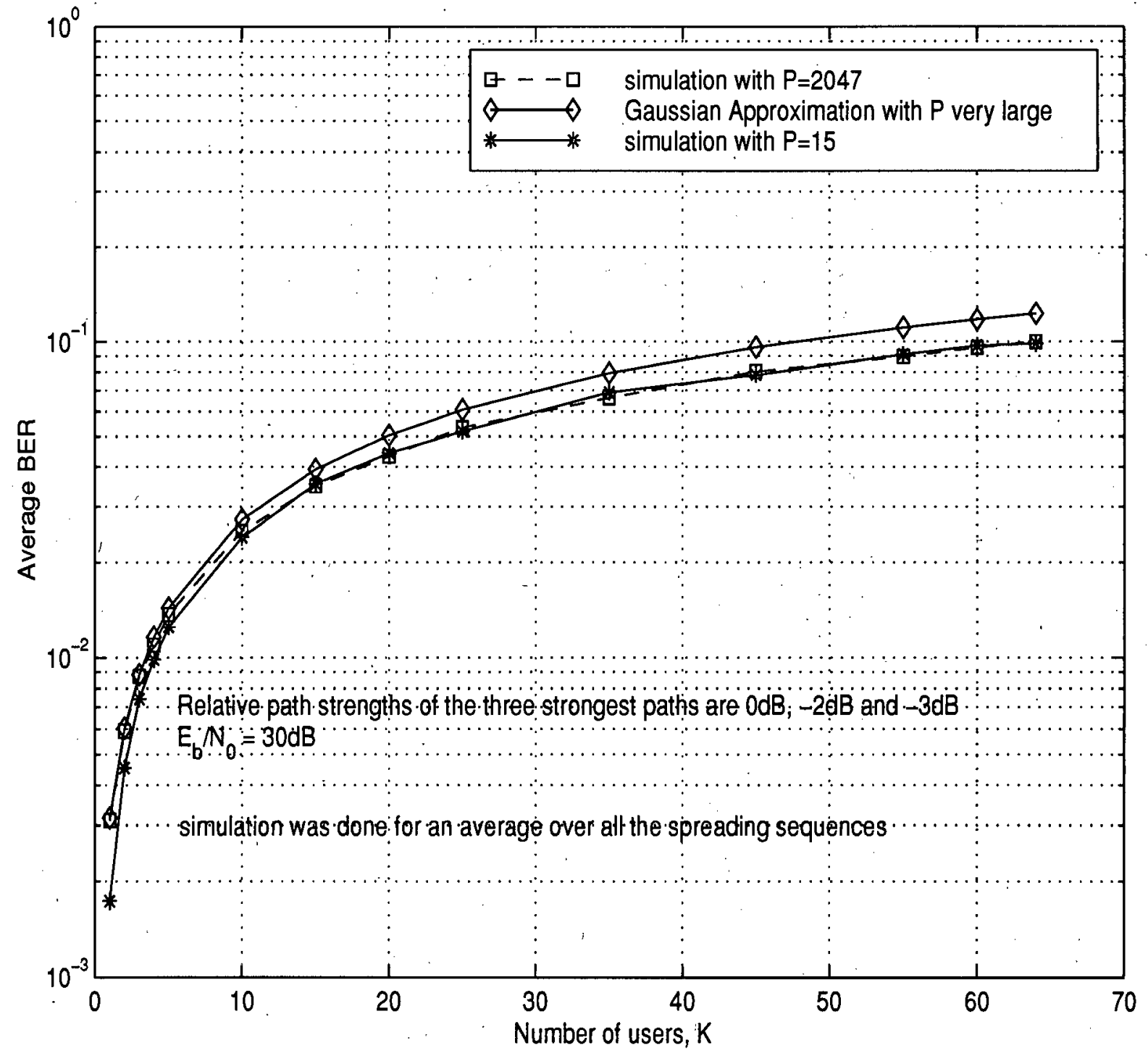

Figure 3.5: BER in a Rayleigh faded channel (with strong paths) using different evaluation techniques, $M=64$ 


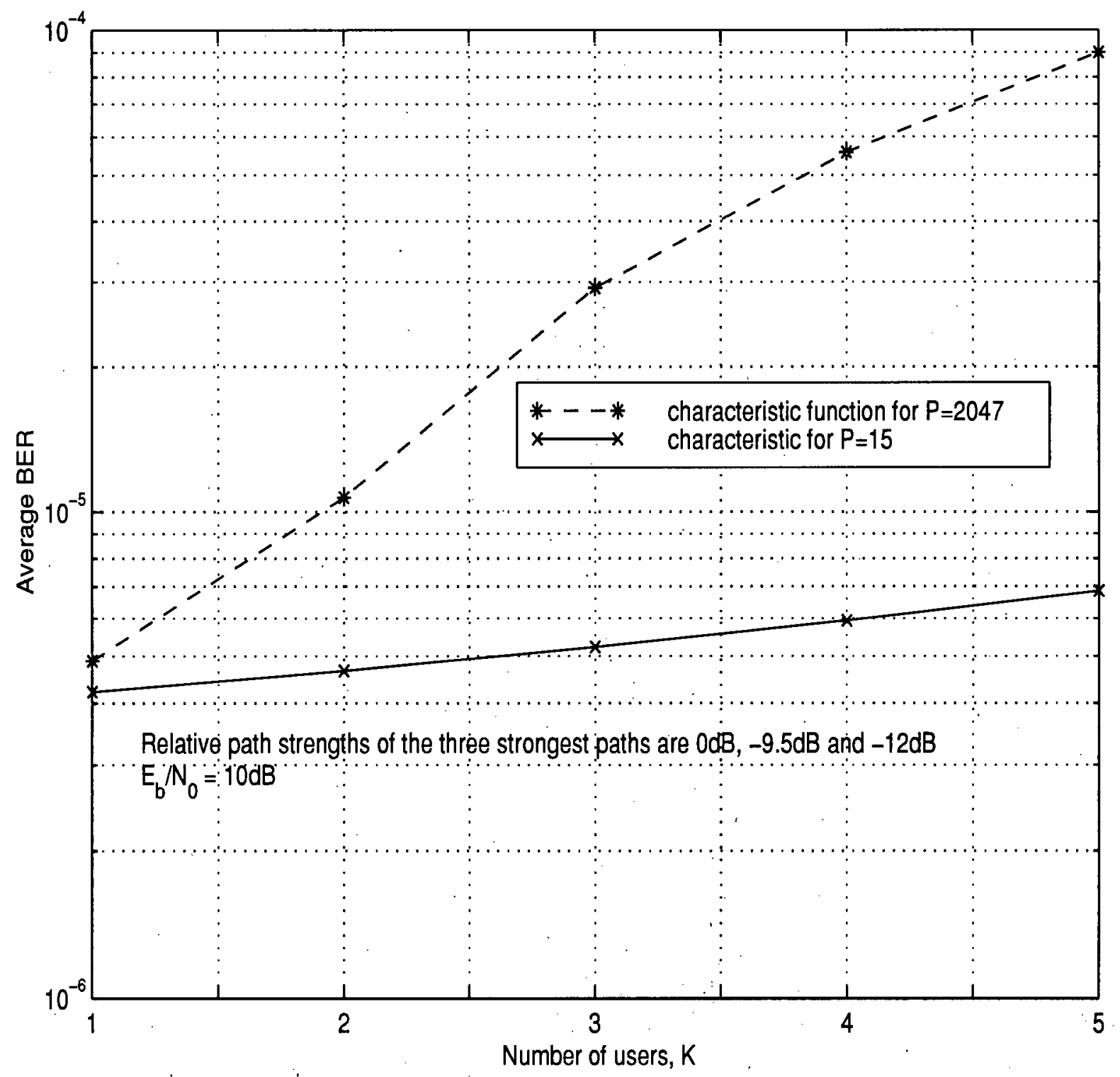

Figure 3.6: BER in a Rician faded channel with weak multipaths, $M=64$ 


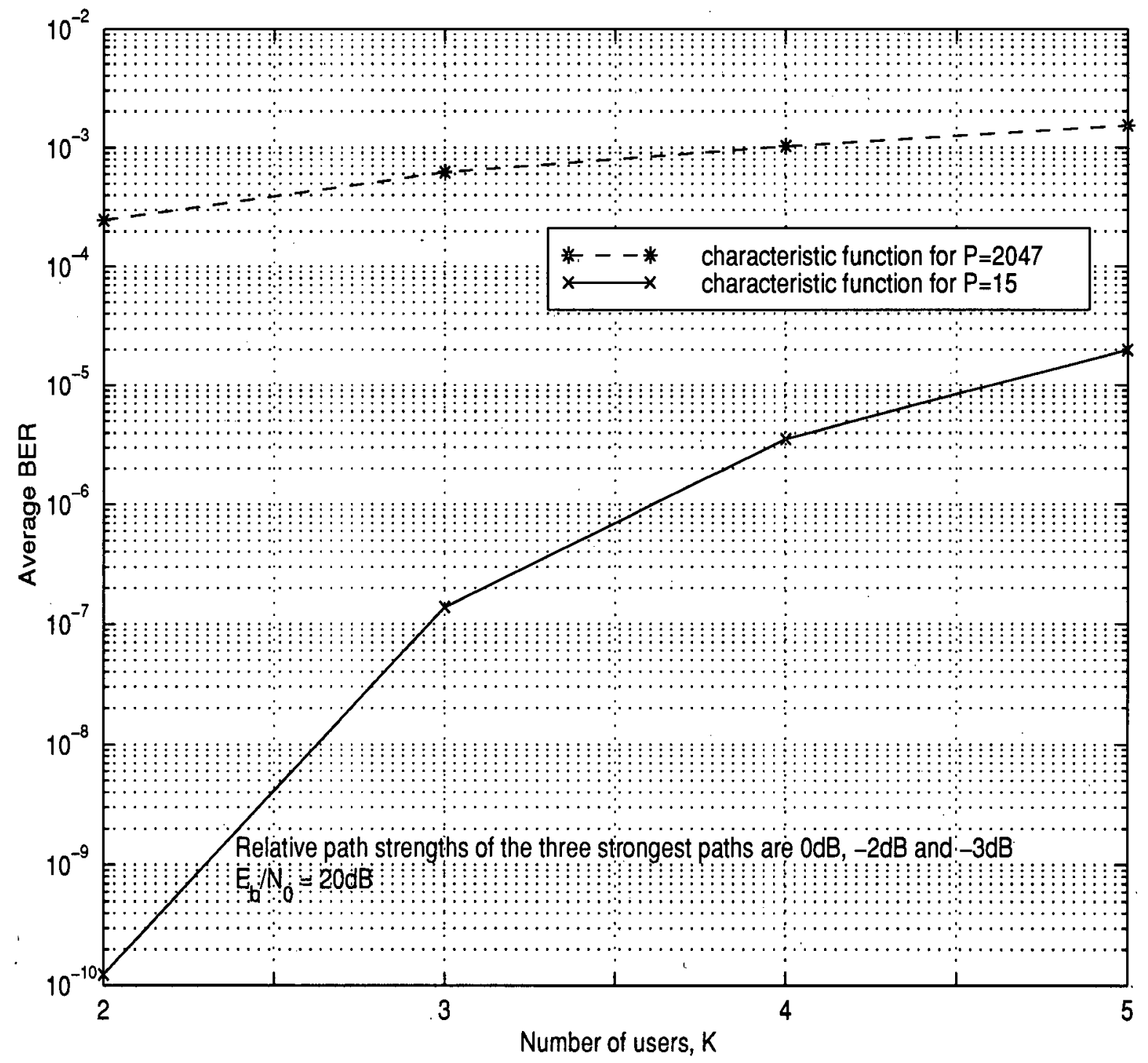

Figure 3.7: BER in a Rician faded channel with strong multipaths, $M=64$ 


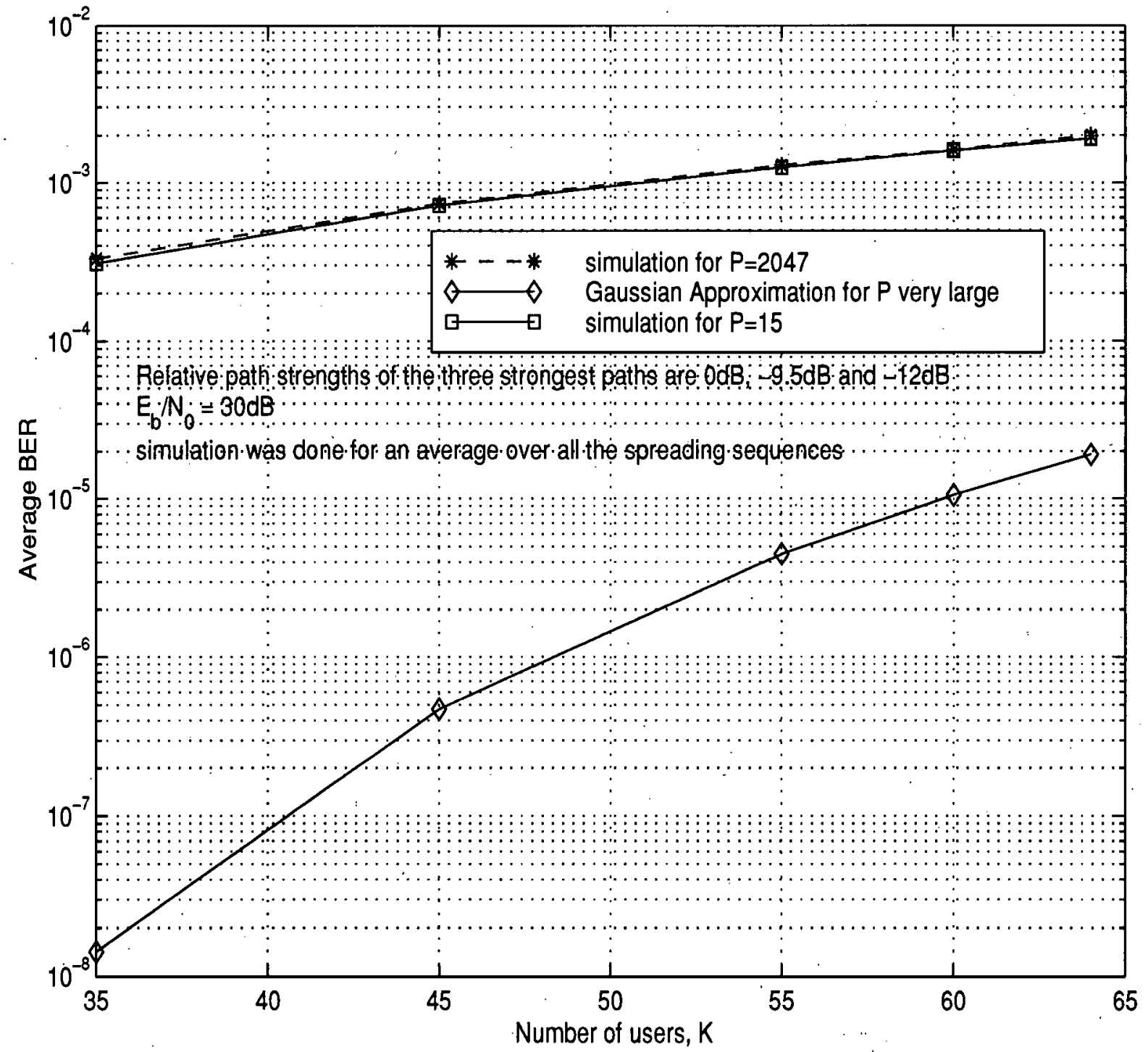

Figure 3.8: BER in a Rician faded channel (with weak paths) using different evaluation techniques, $M=64$ 


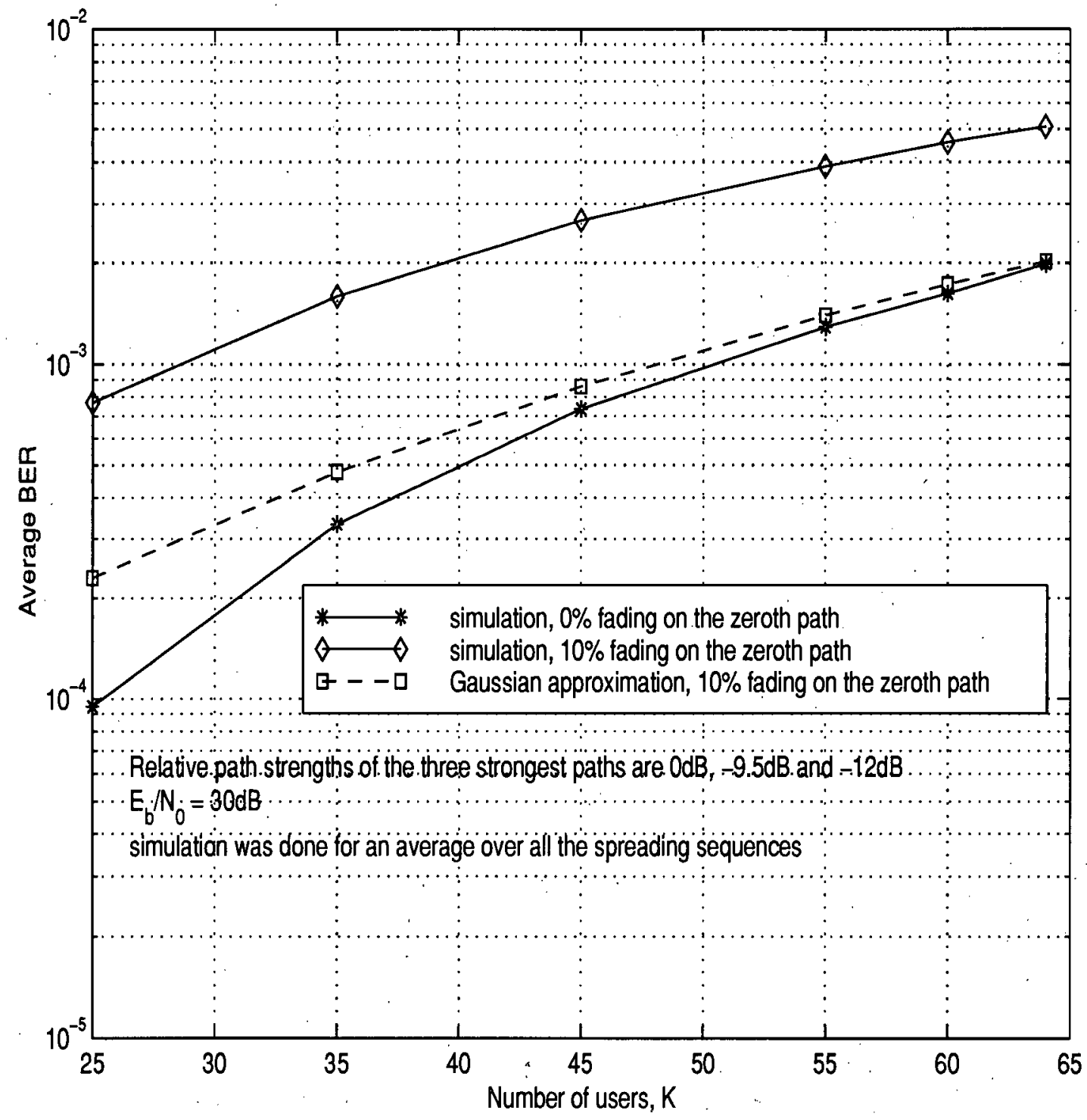

Figure 3.9: BER in a Rician faded channel (with weak paths and $10 \%$ fading power on the zeroth path) using different evaluation techniques, $M=64$ 


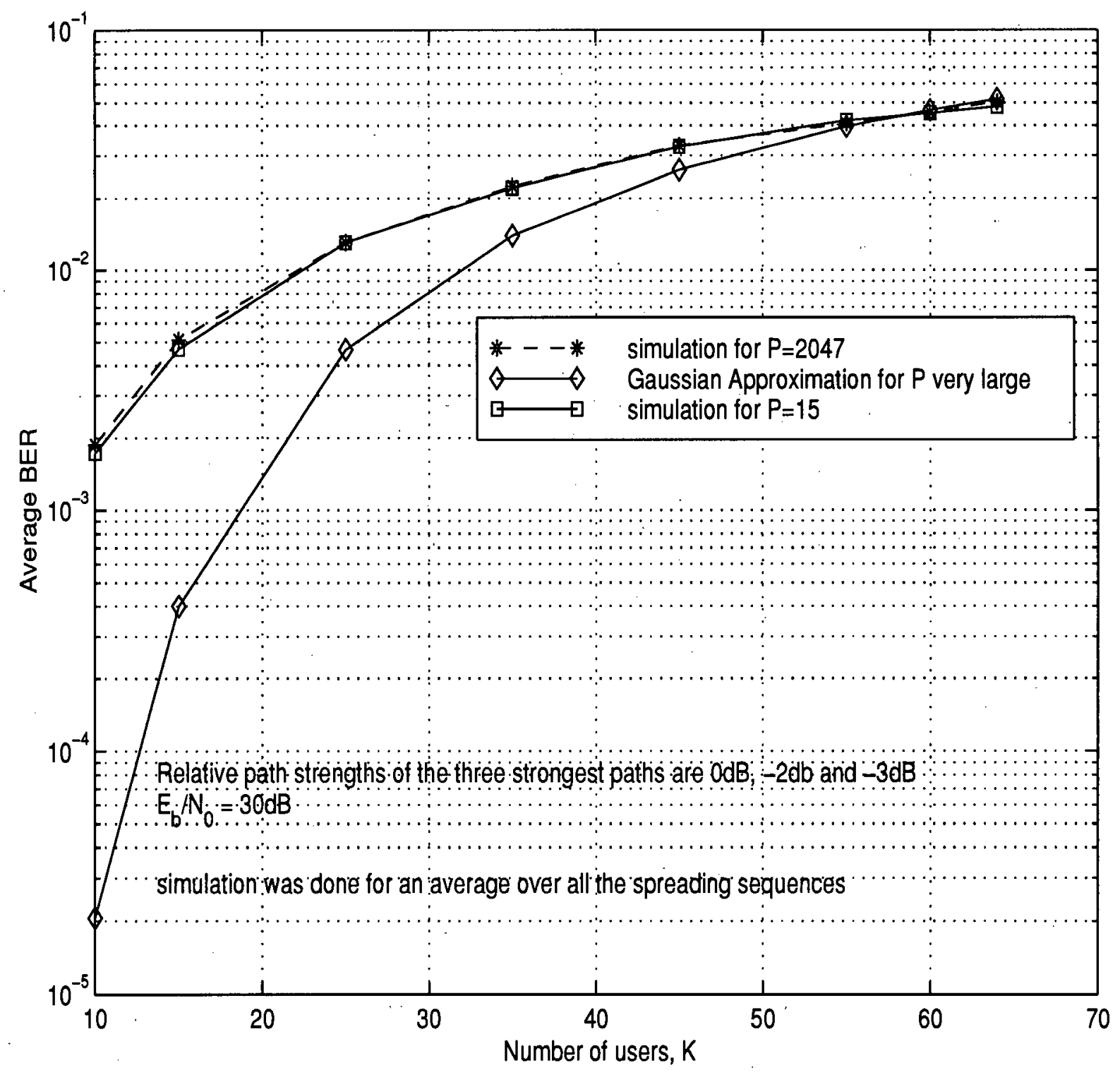

Figure 3.10: BER in a Rician faded channel (with strong paths) using different evaluation techniques, $M=64$ 
(a) LINEAR SCALE

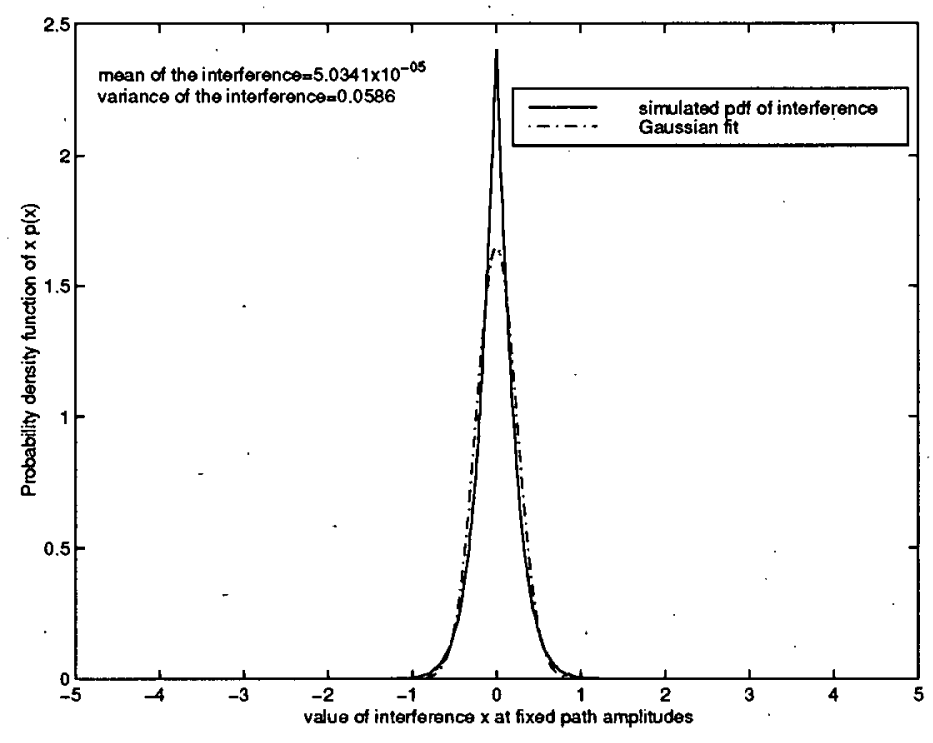

(b) LOGARITHMIC SCALE

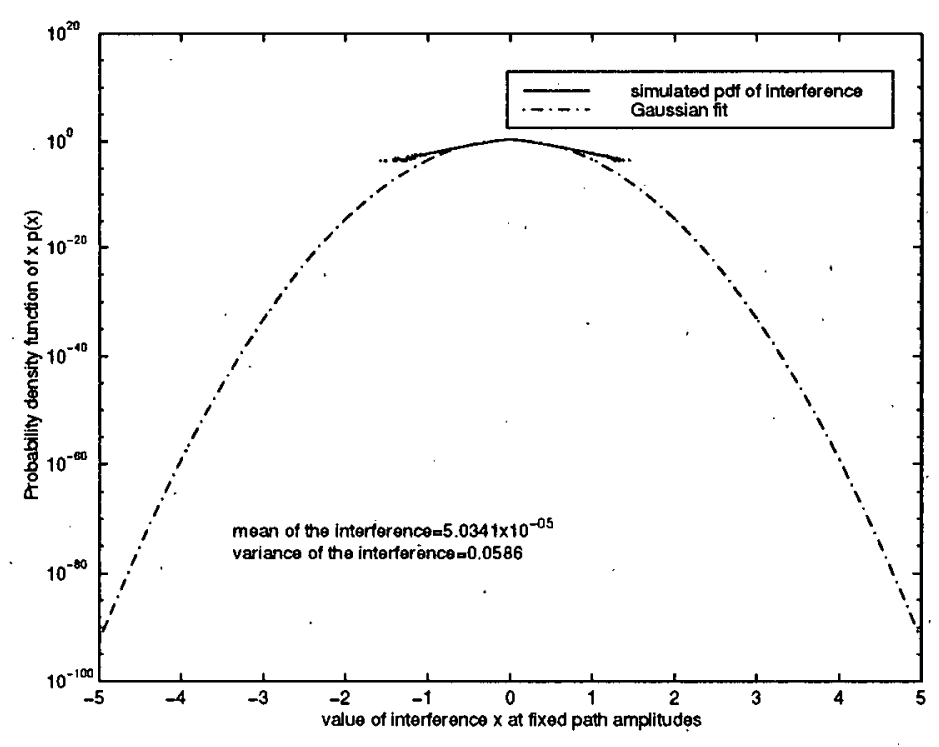

Figure 3.11: Probability density function of the multipath interference (weak multipaths) when $\beta=1.0,\left|\alpha_{1}\right|=\gamma_{1},\left|\alpha_{2}\right|=\gamma_{2}$ and $\gamma_{0}=0$ 


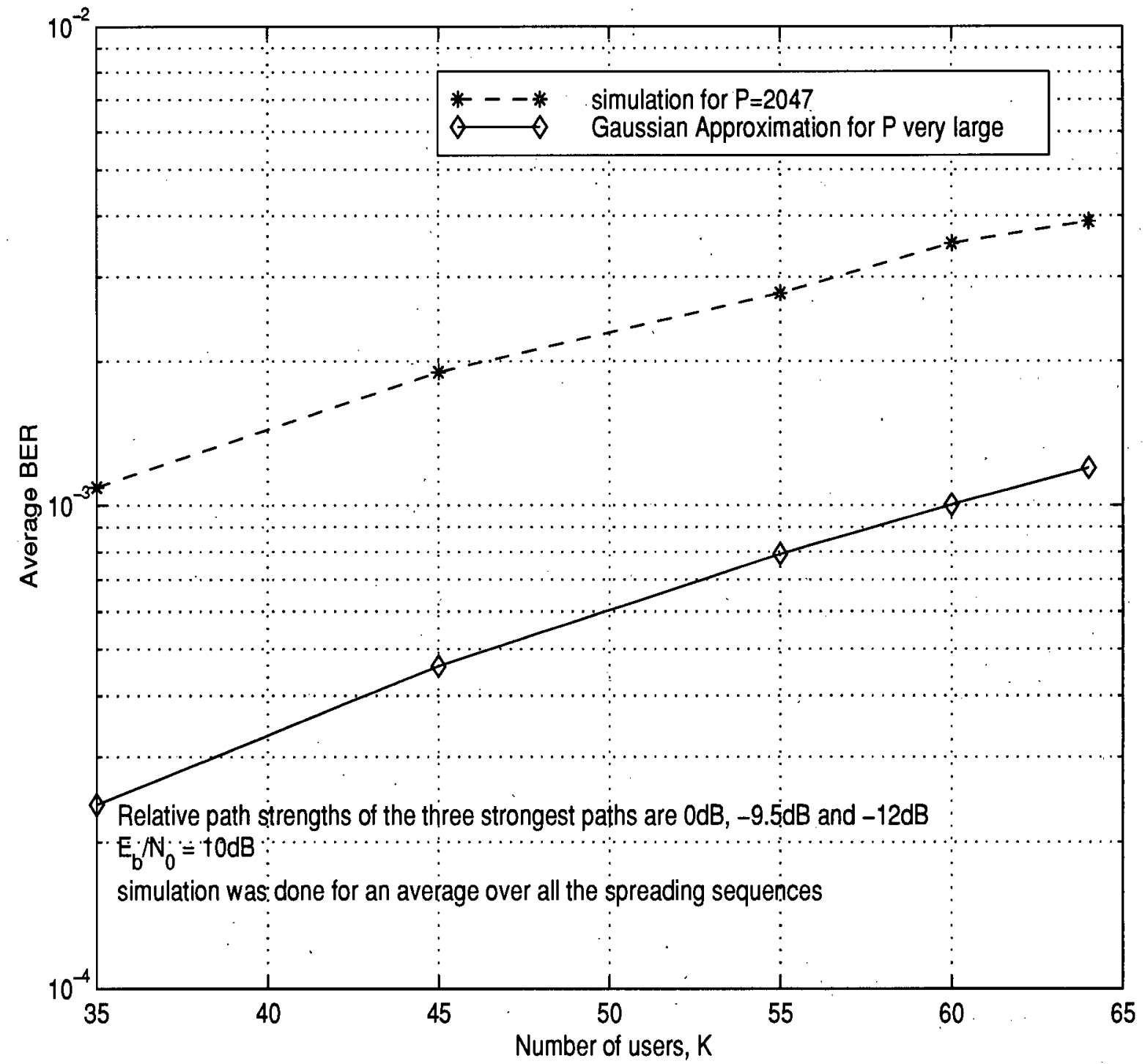

Figure 3.12: BER in a Rician faded channel (with weak paths) using different evaluation techniques, $M=64$ 


\section{Chapter 4}

\section{Multipath Diversity}

\subsection{Introduction}

The performance of wireless systems generally degrades with the presence of multipath signals [9]. One advantage of the wideband nature of CDMA systems is not just being able to discriminate against multipath signals, but being able to coherently combine information from the different paths in order to improve overall performance.

In this chapter, we study possible improvements to the BER of the forward link of CDMA systems using the orthogonal Walsh/PN spreading sequences by using multipath diversity. Generally, in spread spectrum systems, interference in the output of a finger may not be independent of the interference in the output of other fingers. When the time separation between the signals in the paths is more than a bit duration, there will be no overlap between the different portions of the received signals that are involved in the multipath interferences that are in the output of the fingers. However, when the time separation between the signals in two paths is less than a bit duration, there will be an overlap in the portions of the received signals that result in multipath interferences. For the channel multipath model in [14], it is shown that the multipath interference power due to paths whose signals are separated in time by less than a bit duration is higher than that due to paths whose signals are separated by more than a bit duration. In addition, the smaller the separation in time between these signals, the higher is this interference power. The BER obtained with multipath signals that are modeled with time separation 
of between one and two chip durations will tend to provide an upper bound on the BER for systems utilizing multipath diversity. It should be noted that because of the overlap between the portions of the signals that result in the multipath interferences in the different fingers, an analysis technique such as the characteristic function approach becomes quite complicated because of the dependence between the different multipath interference terms.

In this chapter, we base our analysis on the Gaussian approximation, and compare our results to those obtained from simulation with the same channel parameters as used in Chapter 3. First, we present the system and a modified channel model, then we derive expressions for the variance of the multipath interference when maximal ratio combining (Rake receiver) is used. Finally, we present numerical results.

\subsection{System and Channel Model}

The system is made up of $K$ transmitters at the base station, and we assume that there is only one cell. The transmitters are modeled as in Chapter 3. The channel model is also the same as in Chapter 3, except for the modification that the signals that are synchronized to the diversity fingers are pairwise resolvable. Thus, $\tau_{n+1}-\tau_{n} \geq T_{c}$.

The Rake receiver has $B+1$ fingers. These fingers will adaptively search out the $B+1$ strongest signals in the multipaths. We assume that the fadings on these paths are slow enough for the searching mechanism in these fingers to be able to determine the amplitude gains of the signals, and also that the fingers are able to synchronize the despreading sequences to the spreading sequences of the signals in these paths. We base all our analyses on the delayed received signal configuration of the Rake receiver for binary antipodal signaling $[9,28]$ shown in Figure 4.1. We now derive expressions for the mean and the variance of the output decision variable $U_{i}$, and hence obtain BER 


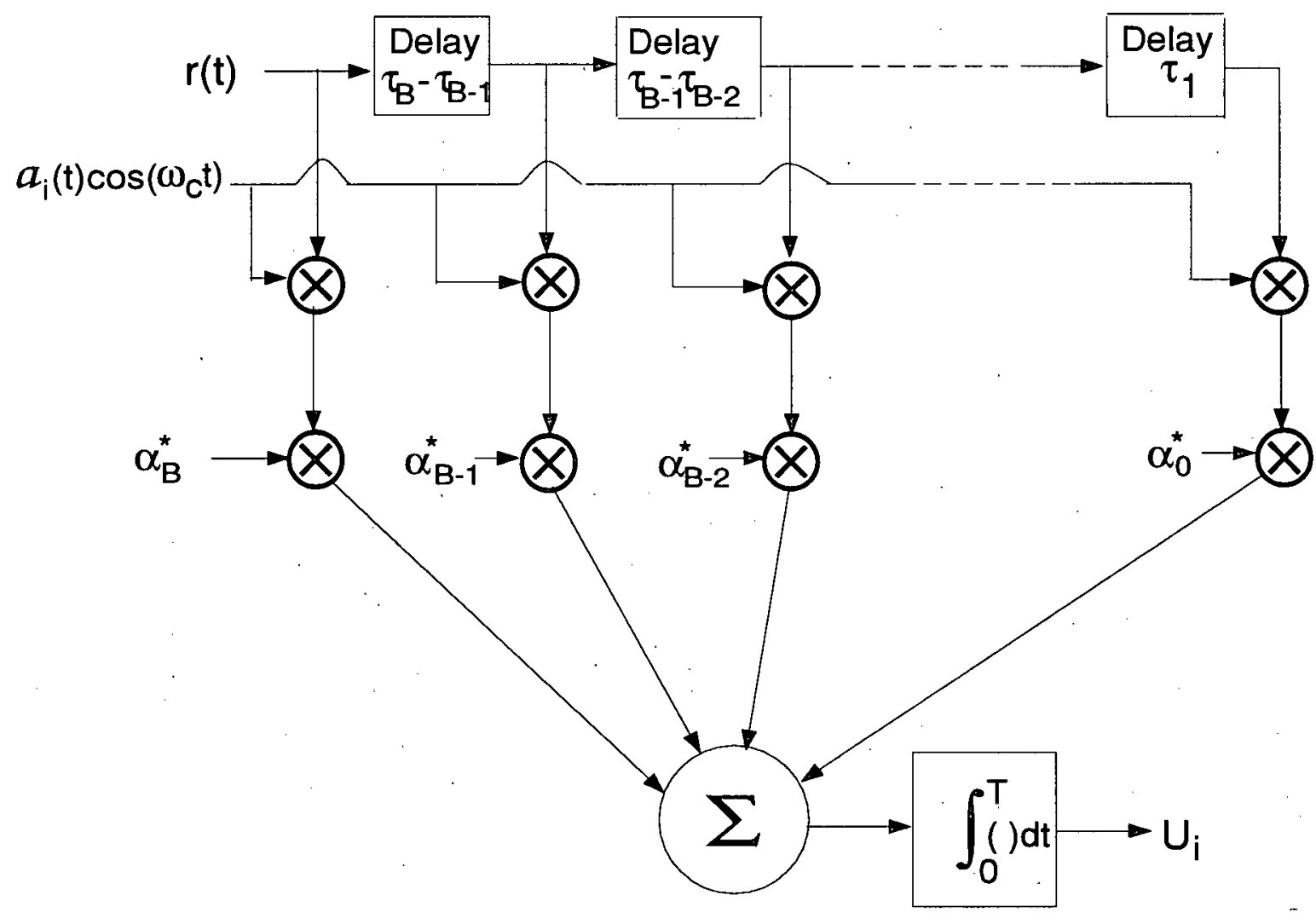

Figure 4.1: Rake Receiver (delayed received signal configuration)

expressions for channels with Rayleigh fading on the zeroth path, and channels with Rician fading on the zeroth path.

\subsection{BER for channels with Rayleigh fading on the zeroth path}

Using the complex representation of the transmitted signal in (3.3), the output decision variable from the Rake receiver can be expressed as

$$
U_{i}=\sum_{n=0}^{B} \int_{\tau_{n}}^{T+\tau_{n}} \operatorname{Re}\left\{\hat{\alpha}_{n}^{*}\left(y_{c}(t)+n(t)\right)\right\} a_{i}\left(t-\tau_{n}\right) \cos \left(\omega_{c}\left(t-\tau_{n}\right)\right) d t
$$




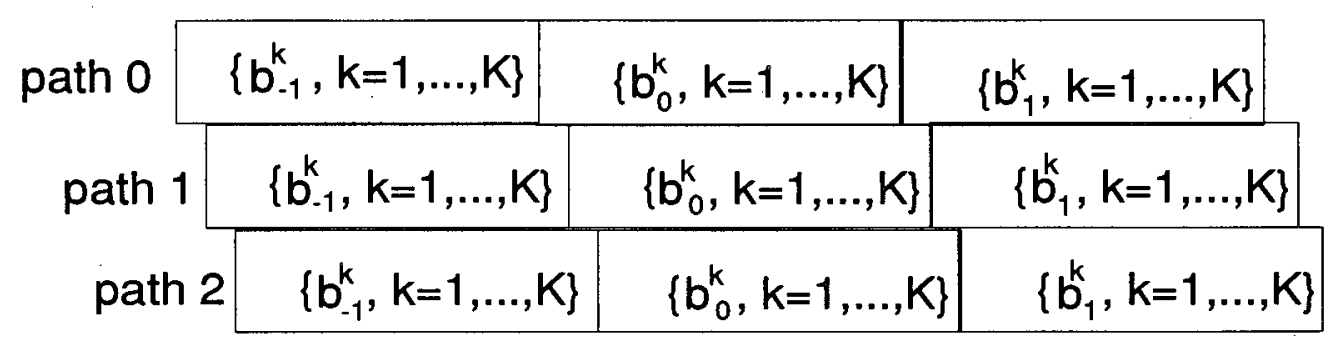

Figure 4.2: Bits affected by multipath interference in three path channel

where $\hat{\alpha}_{n}^{*}$ is the conjugate of the estimated complex gain coefficient of the signal on the $n$th path, and $a_{i}(t)$ is the despreading sequence. If we assume perfect estimates, i.e. $\hat{\alpha}_{n}^{*}=\alpha_{n}^{*}$, the output decision variable can be expressed as

$$
U_{i}=\sqrt{\frac{\mathcal{P}}{2}} T\left\{b_{0}^{i} \sum_{n=0}^{B}\left|\alpha_{n}\right|^{2}+I_{m}+F_{m}\right\}+n^{\prime}
$$

where $I_{m}$ is again the total interference due to the signals simultaneously transmitted by the base station to the other mobiles. For a channel where the signal on the zeroth path has Rayleigh fading, $I_{m}=0 . F_{m}$ is the interference due to multipath signals. The parameter $n^{\prime}$ due to AWGN is obtained from (4.1) as

$$
n^{\prime}=\sum_{n=0}^{B} \int_{\tau_{n}}^{T+\tau_{n}} \operatorname{Re}\left\{\alpha_{n}^{*} n(t)\right\} a_{i}\left(t-\tau_{n}\right) \cos \left(\omega_{c}\left(t-\tau_{n}\right)\right) d t .
$$

To derive expressions for the multipath interference, we consider the information bits that are affected because of the overlapping portions of the signal during correlation in the different fingers. Figure 4.2 shows the bits involved in the multipath interference when the maximum difference between the transmission delays of the paths is less than a bit duration.

Consequently, the multipath interference is obtained by using (3.17), and is given by

$$
F_{m}=\frac{1}{T} \sum_{n=0}^{B} \sum_{\substack{l=0 \\ l \neq n}}^{L} \sum_{\substack{k=1 \\ K}}^{K}\left|\alpha_{n} \alpha_{l}\right|\left[b_{l 1}^{k} R_{k, i}\left(s, \tau_{n l}\right)+b_{l 2}^{k} \hat{R}_{k, i}\left(s, \tau_{n l}\right)\right] \cos \left(\phi_{n l}\right)
$$


In (4.4), $b_{l 1}^{k}$ and $b_{l 2}^{k}$ denote the two adjacent bits of the $k$ th user in the signal in path $l$ that interfere with the desired bit in path $n$. If the signal in path $l$ is delayed in time with respect to that in path $n$, the bits are $b_{-1}^{k}$ and $b_{0}^{k}$ respectively. If the signal in path $l$ is advanced in time with respect to that in path $n$, the bits are $b_{0}^{k}$ and $b_{1}^{k}$ respectively. The parameters $\phi_{n l}=\phi_{n}-\phi_{l}$ and $\tau_{n l}=\left|\tau_{n}-\tau_{l}\right|$ represent the carrier phase difference and the relative delay of the signals in path $n$ and path $l$ respectively.

\subsubsection{Gaussian approximation}

To obtain the BER via the Gaussian approximation technique, we need to obtain the variance of the output of the decision variable. Conditioned on the complex amplitude gains $\alpha_{0}, \cdots, \alpha_{L}, n^{\prime}$ is independent of $F_{m}$, and the variance of $U_{i}$ is given by

$$
\operatorname{var}\left(U_{i}\right)=\operatorname{var}\left(n^{\prime}\right)+\operatorname{var}\left(F_{m}\right) \frac{\mathcal{P} T^{2}}{2}
$$

where $\operatorname{var}\left(n^{\prime}\right)$ is obtained from (4:3) and is given by [4]

$$
\operatorname{var}\left(n^{\prime}\right)=\frac{N_{0} T}{4} \sum_{n=0}^{B}\left|\alpha_{n}\right|^{2} .
$$

Since both $n^{\prime}$ and $F_{m}$ are zero mean random variables, conditioning on the complex amplitude gain $\alpha_{n}$ and $b_{0}^{i}=1$, the mean of $U_{i}$ is given by

$$
\bar{U}_{i}=\sqrt{\frac{\mathcal{P}}{2}} T \sum_{n=0}^{B}\left|\alpha_{n}\right|^{2} .
$$

The conditional SNR is given by

$$
\operatorname{SNR}\left(\alpha_{0}, \cdots, \alpha_{L}\right)=\frac{\left(\sum_{n=0}^{B}\left|\alpha_{n}\right|^{2}\right)^{2}}{\frac{N_{0}}{2 E_{b}} \sum_{n=0}^{B}\left|\alpha_{n}\right|^{2}+\operatorname{var}\left(F_{m}\right)} .
$$

Assuming the interference + noise is Gaussian, the conditional BER is given by

$$
P_{e i}\left(\alpha_{0}, \cdots, \alpha_{L}\right)=\mathrm{Q}\left(\sqrt{\operatorname{SNR}\left(\alpha_{0}, \cdots, \alpha_{L}\right)}\right) .
$$


The average BER can be obtained by averaging over the complex amplitude gains $\alpha_{0}, \cdots, \alpha_{L}$. Now, we derive expressions for the variance of the multipath interference. We can rewrite $(4.4)$ as

$$
\begin{gathered}
F_{m}=\frac{1}{T} \sum_{n=0}^{B} \sum_{k=1}^{K}\left\{\sum_{l=0}^{n-1}\left|\alpha_{n} \alpha_{l}\right|\left[b_{0}^{k} R_{k, i}^{\prime}\left(s, \tau_{n l}\right)+b_{1}^{k} \hat{R}_{k, i}^{\prime}\left(s, \tau_{n l}\right)\right] \cos \left(\phi_{n l}\right)\right. \\
\left.+\sum_{l=n+1}^{L}\left|\alpha_{n} \alpha_{l}\right|\left[b_{-1}^{k} R_{k, i}\left(s, \tau_{n l}\right)+b_{0}^{k} \hat{R}_{k, i}\left(s, \tau_{n l}\right)\right] \cos \left(\phi_{n l}\right)\right\} \\
\text { where, } \quad R_{k, i}^{\prime}\left(\tau_{n l}\right)=\int_{0}^{T-\tau_{n l}} a_{i}(t) a_{k}\left(t+\tau_{n l}\right) d t \\
\text { and } \quad \hat{R}_{k, i}^{\prime}\left(\tau_{n l}\right)=\int_{T-\tau_{n l}}^{T} a_{i}(t) a_{k}\left(t+\tau_{n l}\right) d t
\end{gathered}
$$

The variance of the multipath interference is given by

$$
\begin{aligned}
\operatorname{var}\left(F_{m}\right)= & \frac{1}{T^{2}} \mathrm{E}\left[\left(\sum _ { n = 0 } ^ { B } \sum _ { k = 1 } ^ { K } \left\{\sum_{l=0}^{n-1}\left|\alpha_{n} \alpha_{l}\right|\left[b_{0}^{k} R_{k, i}^{\prime}\left(s, \tau_{n l}\right)+b_{1}^{k} \hat{R}_{k, i}^{\prime}\left(s, \tau_{n l}\right)\right] \cos \left(\phi_{n l}\right)\right.\right.\right. \\
& \left.\left.\left.+\sum_{l=n+1}^{L}\left|\alpha_{n} \alpha_{l}\right|\left[b_{-1}^{k} R_{k, i}\left(s, \tau_{n l}\right)+b_{0}^{k} \hat{R}_{k, i}\left(s, \tau_{n l}\right)\right] \cos \left(\phi_{n l}\right)\right\}\right)^{2}\right]
\end{aligned}
$$

Since $\mathrm{E}\left[b_{n}^{i} b_{m}^{k}\right]=0$ for $i \neq k$ and for all $n$ and $m$, we can simplify (4.12) to

$$
\operatorname{var}\left(F_{m}\right)=\frac{1}{T^{2}} \sum_{k=1}^{K} \mathrm{E}\left[\left(\sum_{n=0}^{B}\left\{\sum_{l=0}^{n-1} X_{n l k}+\sum_{l=n+1}^{L} Y_{n l k}\right\}\right)^{2}\right]
$$

where

$$
\begin{aligned}
X_{n l k} & =\left|\alpha_{n} \alpha_{l}\right|\left[b_{0}^{k} R_{k, i}^{\prime}\left(s, \tau_{n l}\right)+b_{1}^{k} \hat{R}_{k, i}^{\prime}\left(s, \tau_{n l}\right)\right] \cos \left(\phi_{n l}\right) \\
\text { and } \quad Y_{n l k} & =\left|\alpha_{n} \alpha_{l}\right|\left[b_{-1}^{k} R_{k, i}\left(s, \tau_{n l}\right)+b_{0}^{k} \hat{R}_{k, i}\left(s, \tau_{n l}\right)\right] \cos \left(\phi_{n l}\right)
\end{aligned}
$$

Expanding the square, we have

$$
\begin{aligned}
\operatorname{var}\left(F_{m}\right)= & \frac{1}{T^{2}} \sum_{k=1}^{K}\left\{\sum_{n=0}^{B} \mathrm{E}\left[\left(\left\{\sum_{l=0}^{n-1} X_{n l k}+\sum_{l=n+1}^{L} Y_{n l k}\right\}\right)^{2}\right]+2 \sum_{n=0}^{B-1} \sum_{m=n+1}^{B}\right. \\
& \left.\mathrm{E}\left[\left(\sum_{l=0}^{n-1} X_{n l k}+\sum_{l=n+1}^{L} Y_{n l k}\right)\left(\sum_{j=0}^{m-1} X_{m j k}+\sum_{j=m+1}^{L} Y_{m j k}\right)\right]\right\} .
\end{aligned}
$$


The cross product terms in (4.12) disappear except when $l=m$ and $j=n$. Since $\mathrm{E}\left[\cos \left(\phi_{n l}\right) \cos \left(\phi_{m l}\right)\right]=0$ for $m \neq n$, we have

$$
\operatorname{var}\left(F_{m}\right)=\frac{1}{T^{2}} \sum_{k=1}^{K}\left\{\sum_{n=0}^{B}\left\{\sum_{l=0}^{n-1} \mathrm{E}\left[X_{n l k}^{2}\right]+\sum_{l=n+1}^{L} \mathrm{E}\left[Y_{n l k}^{2}\right]\right\}+2 \sum_{n=0}^{B-1} \sum_{m=n+1}^{B} \mathrm{E}\left[Z_{n m k}\right]\right\}
$$

where

$$
Z_{n m k}=\left|\alpha_{n} \alpha_{m}\right|^{2}\left[R_{k, i}^{\prime}\left(s, \tau_{n m}\right) \hat{R}_{k, i}\left(s, \tau_{n m}\right)\right] \cos ^{2}\left(\phi_{n m}\right)
$$

If the period of the m-sequence is long compared to the period of the Walsh sequence, we can model the sequence as random. This was verified for a period of Walsh sequence, $M$, of 64 and a period of $\mathrm{m}$-sequence of 2047. Consequently, we have

$$
\mathrm{E}\left[X_{n l k}^{2}\right]=\mathrm{E}\left[Y_{n l k}^{2}\right]=\left|\alpha_{n}^{\prime} \alpha_{l}\right|^{2} M T_{c}^{2} / 3
$$

Also,

$$
\begin{aligned}
\mathrm{E}\left[Z_{n m k}^{2}\right] & =\left|\alpha_{n} \alpha_{m}\right|^{2}\left(M-l_{m n}\right) T_{c}^{2} / 3, \quad \text { for } \quad k \doteq i \\
& =0, \quad \text { for } \quad k \neq i
\end{aligned}
$$

where $l_{m n}=\left\lfloor\tau_{m n} / T_{c}\right\rfloor$. Since we model the multipath signal arrival times as clustered, we have an upper bound for the variance if we replace $l_{m n}$ by $(m-n)$. We rewrite (4.16) as

$$
\operatorname{var}\left(F_{m}\right)=\frac{K}{3 M} \sum_{n=0}^{B} \sum_{\substack{l=0 \\ l \neq n}}^{L}\left|\alpha_{n}\right|^{2}\left|\alpha_{l}\right|^{2}+\frac{2}{3 M^{2}} \sum_{n=0}^{B-1} \sum_{m=n+1}^{B}\left|\alpha_{n}\right|^{2}\left|\alpha_{m}\right|^{2}(M-m+n) .
$$

We can simplify the expression for the variance of the multipath interference further by replacing $\left|\alpha_{n}\right|^{2}$ for $n=B+1, \cdots, L$ by their mean values. Thus, we have

$$
\begin{aligned}
\operatorname{var}\left(F_{m}\right)= & \frac{K}{3 M} \sum_{n=0}^{B}\left|\alpha_{n}\right|^{2}\left\{\sum_{\substack{l=0 \\
l \neq n}}^{B}\left|\alpha_{l}\right|^{2}+\sum_{l=B+1}^{L} \gamma_{l}^{2}\right\} \\
& +\frac{2}{3 M^{2}} \sum_{n=0}^{B-1} \sum_{m=n+1}^{B}\left|\alpha_{n}\right|^{2}\left|\alpha_{m}\right|^{2}(M-m+n) .
\end{aligned}
$$


The average BER can be obtained by averaging the conditional BER over the fading power gains $\left|\alpha_{n}\right|^{2}$ for $n=0, \cdots, B$. This can be done using numerical integration or via Monte Carlo averaging. It is useful to note that this approach is much less computationally complex than the Monte Carlo simulation approach described in the next section.

\subsubsection{BER via Monte Carlo Simulation}

The evaluation of the average BER via Monte Carlo simulation is similar to the correlation receiver case. One difference is that for each user $k$, we concatenate the $3 M$ elements of the m-sequence following and including the element at a random starting position, $s$, to three periods of the Walsh sequence allocated to user $k$. This concatenated code is used to spread the bandwidth of three adjacent data pulses of $b_{k}(t)$. with amplitudes $b_{-1}^{k}, b_{0}^{k}$ and $b_{1}^{k}$. The other difference is that at the receiver, $\tau_{n}=n T_{c}+\tau_{n}^{\prime}$ and $\tau_{n+1}=(n+1) T_{c}+\tau_{n+1}^{\prime}$ which are the transmission delays of the signals on the $n$th and $(n+1)$ th path respectively, are generated such that $T_{c} \leq \tau_{n+1}-\tau_{n}<2 T_{c}$.' It should be noted that in obtaining the output decision variable of the Rake receiver using (4.2) and (4.10), we utilize the expressions

$$
\begin{aligned}
R_{k, i}^{\prime}\left(\tau_{n l}\right) & =C_{k, i}\left(-l_{n l}\right) \hat{R}_{\psi}\left(\tau_{n l}^{\prime}\right)+C_{k, i}\left(-l_{n l}-1\right) R_{\psi}\left(\tau_{n l}^{\prime}\right) \\
\text { and } \quad \hat{R}_{k, i}^{\prime}\left(\tau_{n l}\right) & =C_{k, i}\left(M-l_{n l}\right) \hat{R}_{\psi}\left(\tau_{n l}^{\prime}\right)+C_{k, i}\left(M-l_{n l}-1\right) R_{\psi}\left(\tau_{n l}^{\prime}\right) \\
\text { where }{ }^{-} \tau_{n l}^{\prime} & =\tau_{n l}-l_{n l} T_{c} .
\end{aligned}
$$

\subsection{BER for channels with Rician fading on the zeroth path}

In this case, the carrier at the finger assigned to the zeroth path synchronizes with the carrier in the non-faded signal in this path. The output decision variable from the Rake 
receiver, assuming perfect estimate of the signal amplitude gains, can be expressed as

$$
U_{i}=\sqrt{\frac{\mathcal{P}}{2}} T\left\{b_{0}^{i}\left(\beta^{2}+\sum_{n=1}^{B}\left|\alpha_{n}\right|^{2}\right)+I_{m}+F_{m}\right\}+n^{\prime} .
$$

The parameter $I_{m}$ which is the interference due to simultaneous transmission to the mobiles on the same path is non-zero because of the faded signal in the zeroth path. Thus,

$$
I_{m}=b_{0}^{i} \beta \alpha_{0} \cos \left(\phi_{0}\right)
$$

The parameter $n^{\prime}$ now has a variance of

$$
\operatorname{var}\left(n^{\prime}\right)=\frac{N_{0} T}{4}\left(\beta^{2}+\sum_{n=1}^{B}\left|\alpha_{n}\right|^{2}\right) .
$$

We have to modify the multipath interference to

$$
\begin{aligned}
F_{m}= & \frac{1}{T}\left\{\sum_{n=1}^{B} \sum_{\substack{l=1 \\
l \neq n}}^{L} \sum_{k=1}^{K}\left|\alpha_{n} \alpha_{l}\right|\left[b_{l 1}^{k} R_{k, i}\left(s, \tau_{n l}\right)+b_{l 2}^{k} \hat{R}_{k, i}\left(s, \tau_{n l}\right)\right] \cos \left(\phi_{n l}\right)\right. \\
& +\sum_{l=1}^{L} \sum_{k=1}^{K}\left|\beta \alpha_{l}\right|\left[b_{l 1}^{k} R_{k, i}\left(s, \tau_{l}\right)+b_{l 2}^{k} \hat{R}_{k, i}\left(s, \tau_{l}\right)\right] \cos \left(\phi_{l}\right) \\
& +\sum_{n=1}^{B} \sum_{k=1}^{K}\left\{\left|\alpha_{n} \beta\right|\left[b_{01}^{k} R_{k, i}\left(s, \tau_{n}\right)+b_{02}^{k} \hat{R}_{k, i}\left(s, \tau_{n}\right)\right] \cos \left(\phi_{n}\right)\right. \\
& \left.\left.+\left|\alpha_{n} \alpha_{0}\right|\left[b_{01}^{k} R_{k, i}\left(s, \tau_{n}\right)+b_{02}^{k} \hat{R}_{k, i}\left(s, \tau_{n}\right)\right] \cos \left(\phi_{n} 0\right)\right\}\right\}
\end{aligned}
$$

\subsubsection{Gaussian Approximation}

Conditioning on the signal amplitude gains $\beta$ and $\alpha_{0}, \cdots, \alpha_{L}$, the variance of the Rake receiver output decision variable is given by

$$
\operatorname{var}\left(U_{i}\right)=\operatorname{var}\left(n^{\prime}\right)+\left(\operatorname{var}\left(I_{m}\right)+\operatorname{var}\left(F_{m}\right)\right) \frac{\mathcal{P} \dot{T}^{2}}{2}
$$

where $\operatorname{var}\left(I_{m}\right)=\beta^{2} \gamma_{0}^{2} / 2$. Note that we have replaced $\left|\alpha_{0}^{2}\right|$ by its average value of $\gamma_{0}^{2}$. The mean of $U_{i}$ is given by

$$
\bar{U}_{i}=\sqrt{\frac{\mathcal{P}}{2}} T\left(\beta^{2}+\sum_{n=1}^{B}\left|\alpha_{n}\right|^{2}\right)
$$


To determine the SNR, we need to derive the variance of $F_{m}$. We have to modify (4.21) to include the non-faded signal. Consequently, the variance of $F_{m}$ is given by

$$
\begin{aligned}
\operatorname{var}\left(F_{m}\right)= & \frac{K}{3 M}\left\{\sum_{n=1}^{B}\left|\alpha_{n}\right|^{2}\left\{\sum_{\substack{l=1 \\
l \neq n}}^{B}\left|\alpha_{l}\right|^{2}+\sum_{l=B+1}^{L} \gamma_{l}^{2}\right\}+\sum_{n=1}^{B}\left|\alpha_{n}\right|^{2}\left\{\beta^{2}+\left|\alpha_{0}\right|^{2}\right\}\right. \\
& \left.+\beta^{2}\left\{\sum_{l=1}^{B}\left|\alpha_{l}\right|^{2}+\sum_{l=B+1}^{L} \gamma_{l}^{2}\right\}\right\}+\frac{2}{3 M^{2}}\left\{\sum_{n=1}^{B-1} \sum_{m=n+1}^{B}\left|\alpha_{n}\right|^{2}\left|\alpha_{m}\right|^{2}(M-m+n)\right. \\
& \left.+\sum_{m=1}^{B} \beta^{2}\left|\alpha_{m}\right|^{2}(M-m)\right\} .
\end{aligned}
$$

The average BER can be obtained by averaging the conditional BER over the complex amplitude gains $\alpha_{0}, \cdots, \alpha_{B}$ using the same technique as in the case for the channel with Rayleigh fading on the zeroth path's signal. The conditional BER is given by

$$
P_{e i}\left(\alpha_{0}, \cdots, \alpha_{L}\right)=\mathrm{Q}\left(\sqrt{\operatorname{SNR}\left(\alpha_{0}, \cdots, \alpha_{L}\right)}\right)
$$

where

$$
\operatorname{SNR}\left(\alpha_{0}, \cdots, \alpha_{L}\right)=\frac{\left(\beta^{2}+\sum_{n=1}^{B}\left|\alpha_{n}\right|^{2}\right)^{2}}{\frac{N_{0}}{2 E_{b}}\left(\beta^{2}+\sum_{n=1}^{B}\left|\alpha_{n}\right|^{2}\right)+\operatorname{var}\left(I_{m}\right)+\operatorname{var}\left(F_{m}\right)} .
$$

\subsubsection{BER via Monte Carlo Simulation}

The procedure to evaluate of the average BER via Monte Carlo simulation is similar to that in Section 4.3.2. The only difference is that we have to take into consideration the presence of the specular signal, and assume that the carrier signal in the finger assigned to the zeroth path is synchronized to the carrier of this specular signal.

\subsection{A Modified Diversity Receiver for Environments with weak multipath signals}

Simulation results show that the performance of the Rake receiver in channels with Rician fading on the zeroth path degrades faster with the number of users in the system when 
the multipath signals (other than the specular signal) are weak compared to when the multipath signals are strong. The explanation for this is that when diversity is applied, the strong zeroth path signals become interference to the weak signals on the other paths. When the number of users increases, a larger amount of interference power is added by virtue of the multipath combination compared to the amount of desired signal power. At some point, the Rake receiver performs worse compared to the conventional correlation receiver.

To be able to utilize the information in the diversity fingers as well as combat the above drawback in the Rake receiver, we modify the Rake receiver. This modified receiver differs from the Rake receiver in the choice of the weights used in the diversity combination. The choice of weights is such that the output of a finger is included in the maximal ratio combining if the fading amplitude of the signal to which the finger. is synchronized is less than the maximum amplitude of the fading multipath signals by a certain threshold. When this threshold is $0 \%$, the diversity combining is similar to sèlection diversity. Figure 4.3 gives a block diagram of the modified Rake receiver. The Threshold Logic unit determines whether the weight to be used to multiply the output of the finger that is synchronized to the $n$th path is zero or $\alpha_{n}^{*}$. For a threshold $\Gamma \%$ modified Rake receiver, this weight is zero if $\left|\alpha_{n}\right|^{2}$ is less than the maximum of the set $\left\{\left|\alpha_{0}\right|^{2}, \cdots,\left|\alpha_{B}\right|^{2}\right\}$ by more than $\Gamma \%$.

\subsection{Numerical Results}

For numerical results, we use the same channel parameters as in Chapter 3 . In all the results, we assume there are $L+1=3$ paths and $B+1=3$ fingers. Also, a processing gain, $M$, of 64 is assumed. We only consider systems with $P=2047$, since for a large number of users, the performance of a system using the concatenated Walsh/m-sequence 


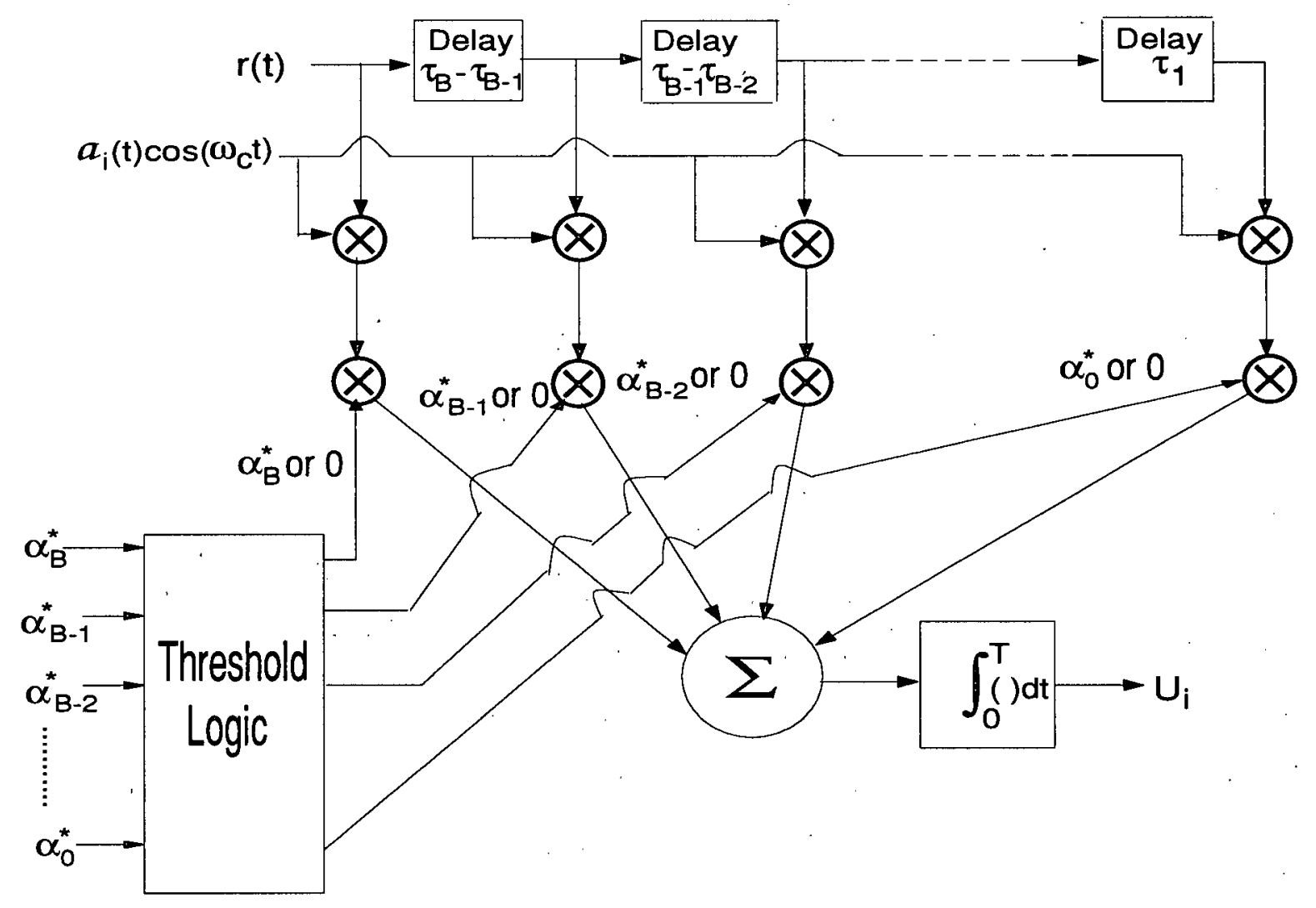

Figure 4.3: Modified Rake Receiver

for bandwidth spreading does not vary much with $P$. The common m-sequence that is used is generated from a shift register with a characteristic polynomial of 5001 in octal notation [29]. Also a transmitted bit energy to noise density ratio $E_{b} / N_{0}$ of $30 \mathrm{~dB}$ is used in all the results presented in this chapter.

Figures 4.4 and 4.5 show the BER performance for the forward link in outdoor channel environments that are characterized by Rayleigh fading on the zeroth path. We observe that the Gaussian approximation is very optimistic for estimating BER. As explained previously, this inaccuracy can be attributed to the dependence in the different random variables involved in the multipath interference. As expected, the Rake receiver provides 
an improved BER over the conventional correlation receiver in the presence of weak multipath signals (Figure 4.4) and strong multipath signals (Figure 4.5).

Figures 4.4 and 4.5 also show the performance of the modified Rake receiver (threshold $0 \%$ and 50\%). In Figures 4.4 and 4.5, the modified Rake receiver (threshold $0 \%$ ) performs worse compared to the Rake receiver and better compared to the correlation receiver. In contrast, Figure 4.4 shows that the modified Rake receiver (threshold 50\%) performs somewhat better compared to the Rake receiver. As expected, its performance does not degrade as fast with the number of users compared to the Rake receiver. Figure 4.5 shows that the modified Rake receiver (threshold $50 \%$ ) gives a similar performance compared to the Rake receiver.

Figures 4.6 and 4.8 show the BER for indoor channel environments that are characterized by Rician fading on the zeroth path. We assume that there is no faded signal in the zeroth path in both figures i.e. $\gamma_{0}=0$. We again observe that the Gaussian approximation is very optimistic for estimating BER. When the powers of the multipath signals are low, we observe from Figure 4.6 that the BER performance obtained from the Rake receiver degrades faster compared to that of the correlation receiver as the number of users increases. This is undesirable since indoor environments are characterized by weak multipath signals even at a bandwidth of up to $20 \mathrm{MHz}$ [21]. Figure 4.8 shows results for channel environments that are characterized by strong multipath signals. In this case, the Rake receiver gives a better BER performance compared to the correlation receiver even for a large number of userś.

Figure 4.7 compares the BER performance of systems operating in channel environments where there is $10 \%$ fading power of the signal transmitted through the zeroth path to one where there is no fading on the zeroth path. It is interesting to note that the BER for channels with $10 \%$ fading doubles in some instances compared to that for channels with $0 \%$ fading on the zeroth path. 
Figures 4.6 and 4.8 also compare the BER performance of the forward link where the modified Rake receiver is used (threshold $0 \%$ and $50 \%$ ) to those where the Rake and the correlation receivers are used. This comparison is done for a channel with weak multipath signals and one with strong multipath signals. In Figure 4.6, we can observe that the modified Rake receiver outperforms both the Rake receiver and the correlation receiver. At a BER of $10^{-3}$, a system using the modified Rake receiver can support between 3 and 4 more users than that using the correlation receiver. Such a system can also support between 7 and 8 more users compared to that using the Rake receiver. In Figure 4.8, we observe that the Rake-receiver is better than the modified Rake receiver (threshold $0 \%$ - selection diversity), and performs similarly to the modified Rake receiver (threshold $50 \%$ ). Note that the plot of the BER performance of the modified Rake receiver (threshold $50 \%$ ) is directly on top of that of the Rake receiver in this figure. Note also that the performance of the modified Rake receiver (threshold $0 \%$ ) lies between that of the Rake receiver and that of the correlation receiver in the channel environment shown in Figure 4.8 .

To examine how the performance of the modified Rake receiver varies with the power in the multipath signals, we present results comparing the BER of the three receivers with the Rice factor as a variable. The Rice factor is defined as the ratio of the power in the specular. signal to the sum of the faded signal average powers. In decibels, the Rice factor $K_{r}$ is given by

$$
K_{r}=10 \log _{10}\left(\frac{\beta^{2}}{\sum_{n=0}^{L} \gamma_{n}^{2}}\right)
$$

Figure 4.9 compares the BER performance of the three receivers for Rice factors ranging from $0 \mathrm{~dB}$ to $9 \mathrm{~dB}$. In the results presented, the average powers of the two faded signals are assumed to be equal. Thus for a channel with a Rice factor of $5 \mathrm{~dB}$, we assume an 
MIP of $0 \mathrm{~dB},-8.01 \mathrm{~dB}$ and $-8.01 \mathrm{~dB}$ for the three path model. We can observe that the Rake receiver starts to perform worse in BER compared to that of the modified Rake receiver (threshold $0 \%$ ) at a Rice factor greater than $5.2 \mathrm{~dB}$. The Rake receiver's BER performance is worse compared to that of the correlation receiver at Rice factor more than around $6 \mathrm{~dB}$. The modified Rake receiver (threshold $50 \%$ ) has a similar performance to the rake receiver at Rice factors between $0 \mathrm{~dB}$ and $2 \mathrm{~dB}$, and performs better than the Rake receiver at Rice factors greater than $2 \mathrm{~dB}$. At Rice factors greater than $7 \mathrm{~dB}$, the modified Rake receiver of threshold $50 \%$ performs slightly worse than the modified Rake of threshold $0 \%$. 


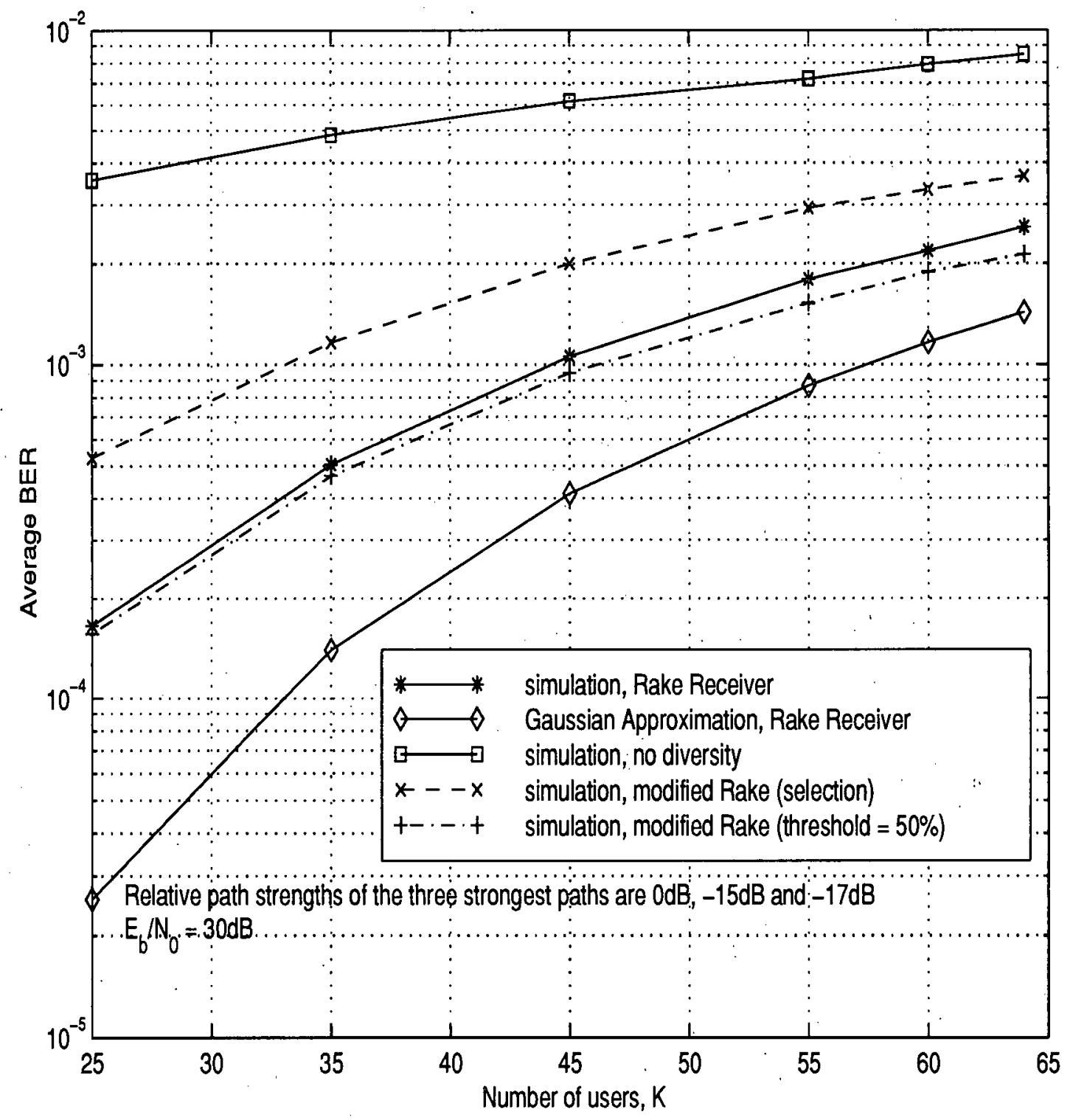

Figure 4.4: BER in a Rayleigh faded channel with weak multipaths, $M=64, P=2047$ (correlation receiver, Rake receiver and modified Rake receiver) 


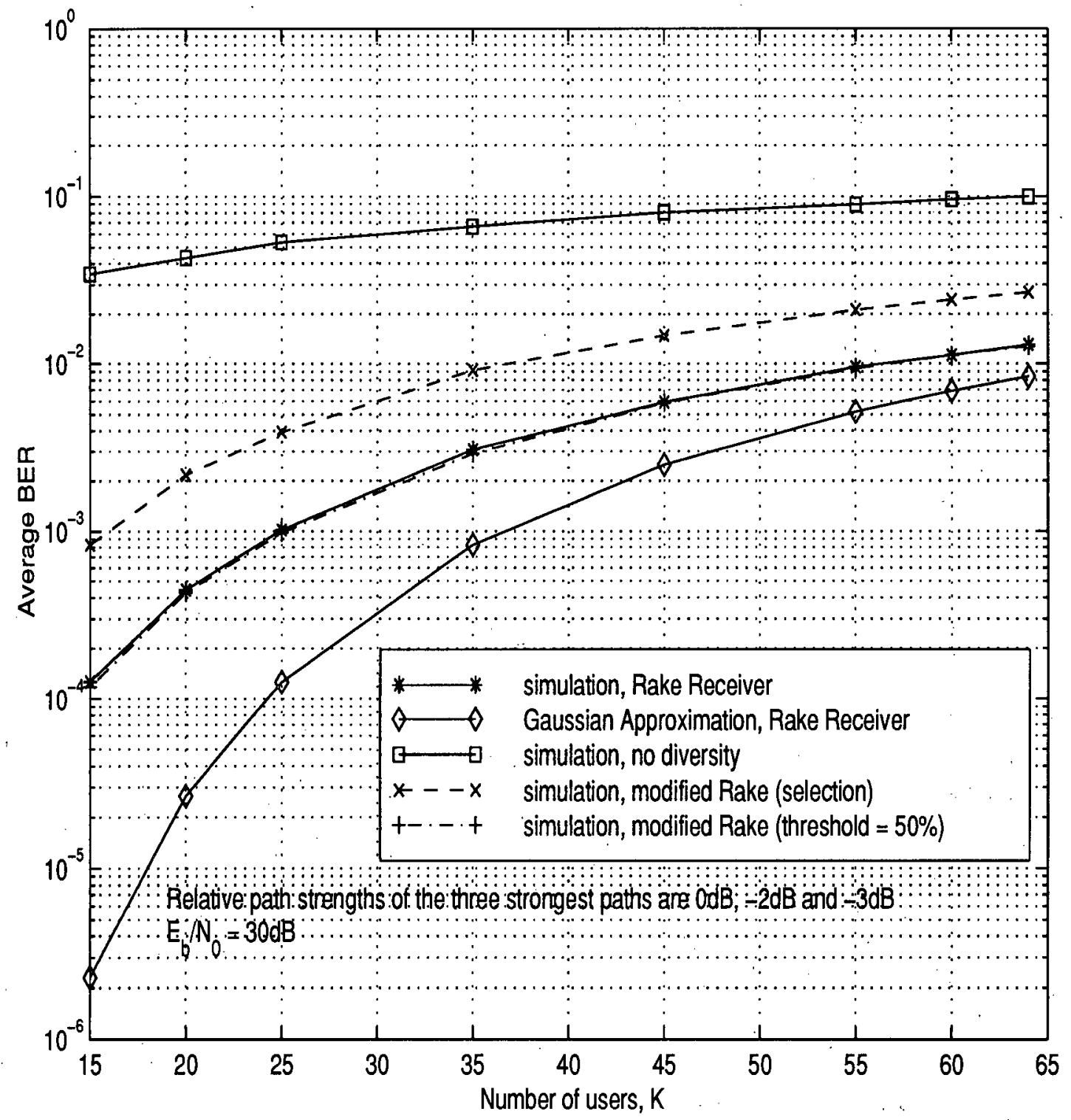

Figure 4.5: BER in a Rayleigh faded channel with strong multipaths, $M=64, P=2047$ (correlation receiver, Rake receiver and modified Rake receiver) 


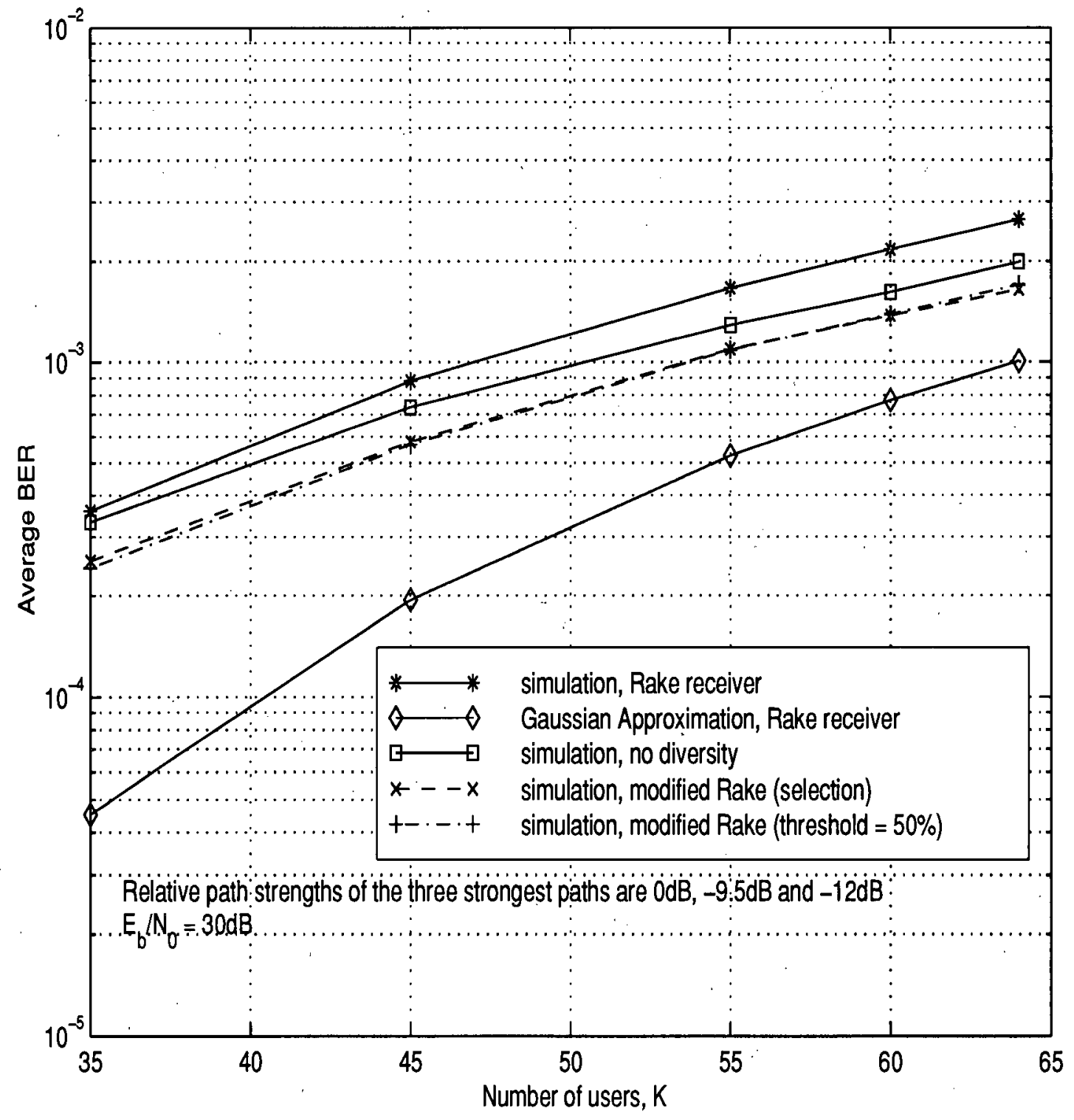

Figure 4.6: BER in a Rician faded channel with weak multipaths, $M=64, P=2047$ (correlation receiver, Rake receiver and modified Rake receiver) 


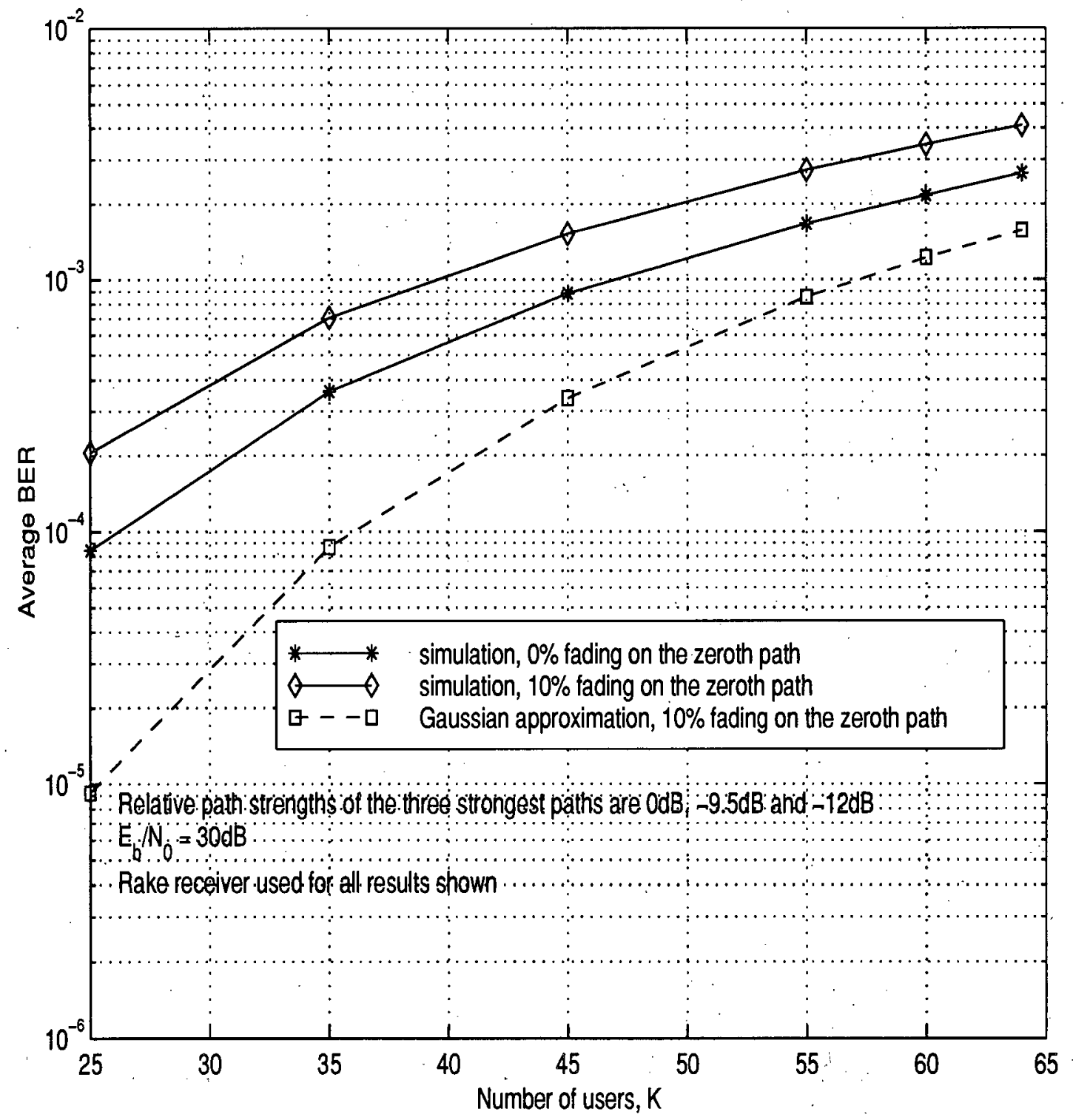

Figure 4.7: BER in a Rician faded channel with weak multipaths and $10 \%$ fading power on the zeroth path, $M=64, P=2047$ (Rake receiver only) 


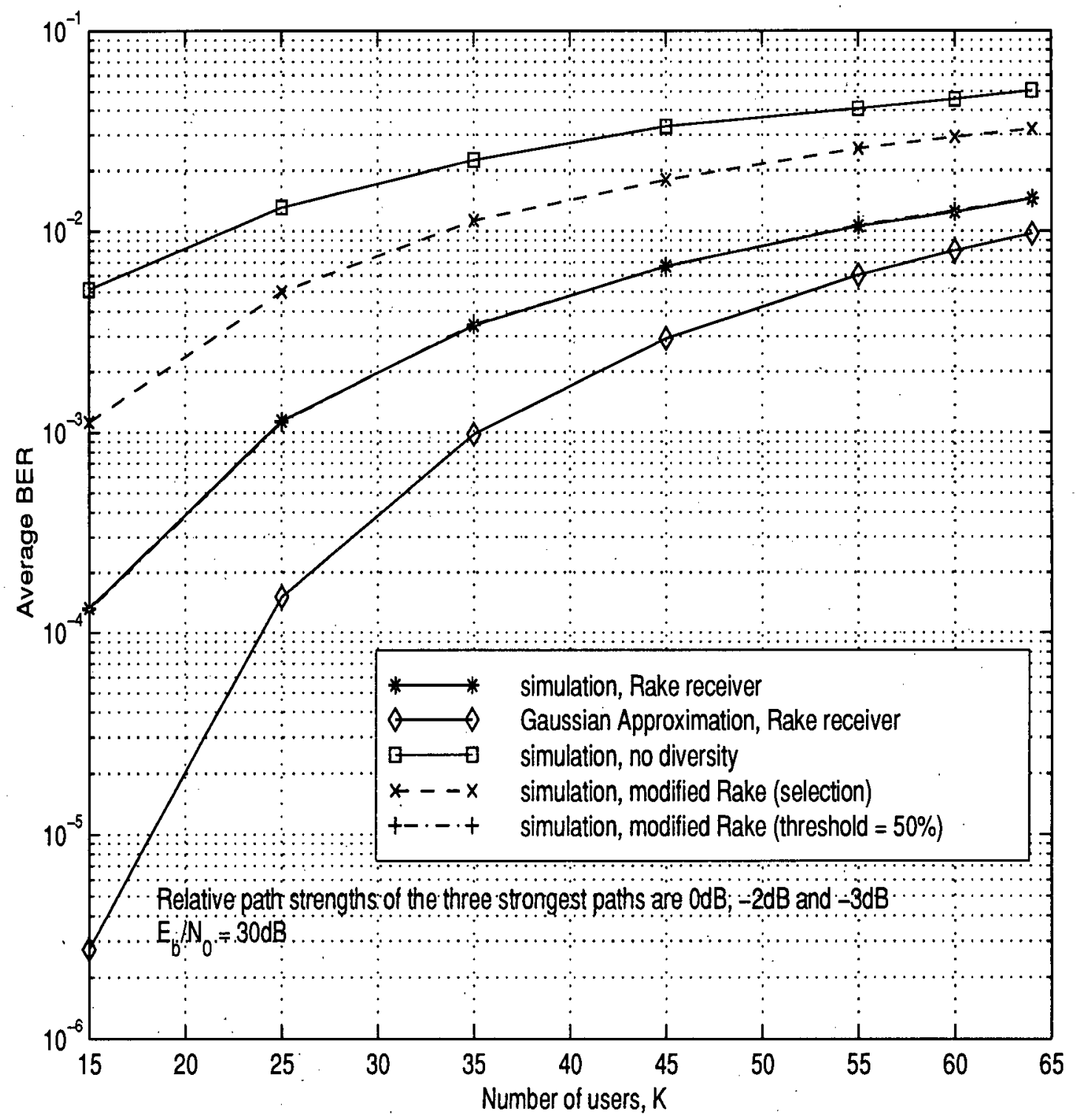

Figure 4.8: BER in a Rician faded channel with strong multipaths, $M=64, P=2047$ (correlation receiver, Rake receiver and modified Rake receiver) 


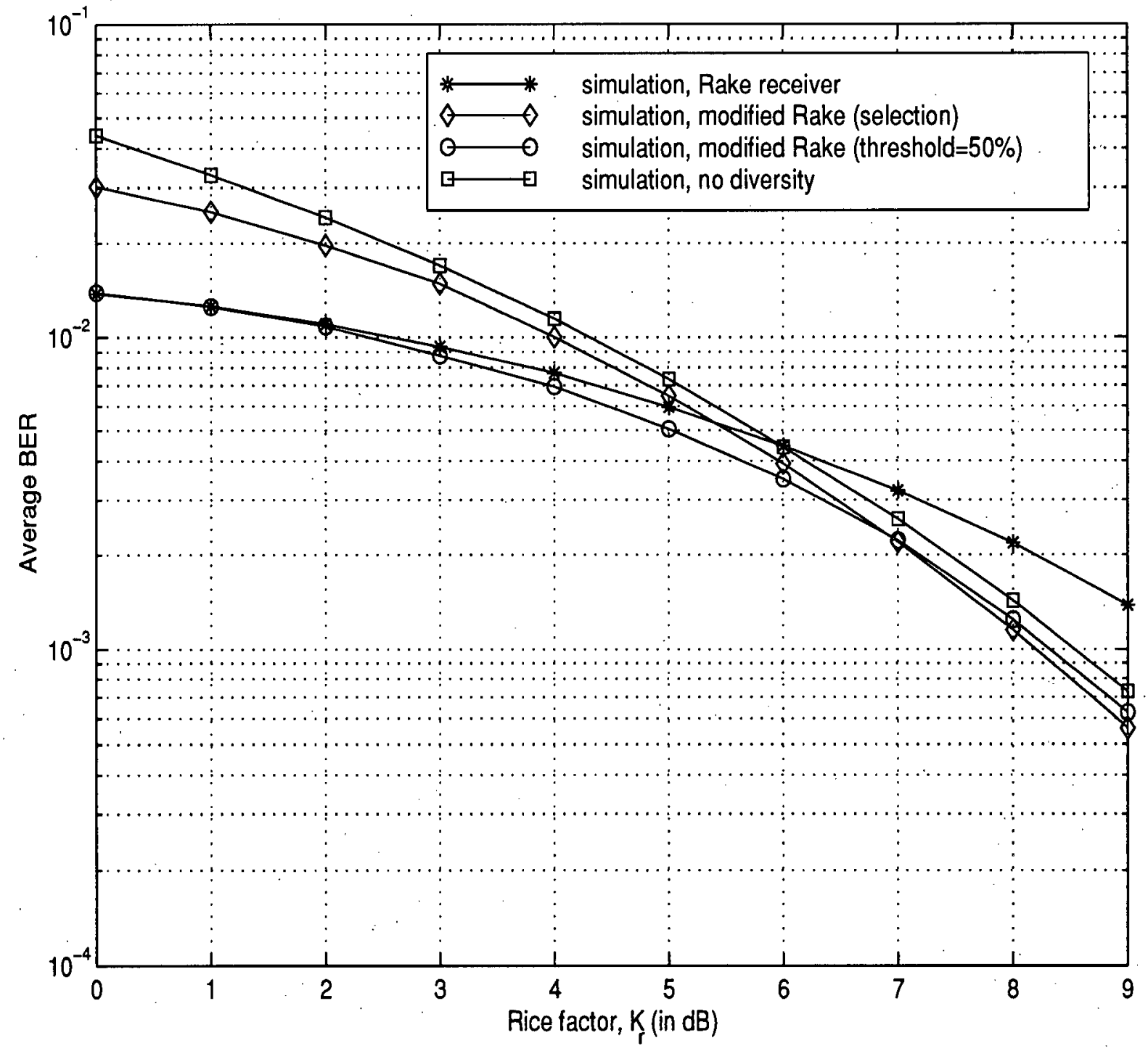

Figure 4.9: BER in a Rician faded channel (with different Rice Factor $K_{r}$ ) using Rake receiver, correlation receiver and the modified Rake receivers; $M=64, P=2047$ and Number of users $K=64$ 


\section{Chapter 5}

\section{Performance on the Forward Link in a Multicell Environment}

\subsection{Introduction}

One of the advantages of CDMA systems is universal frequency reuse. This is the ability to use a common carrier frequency in a multicell area. Since the interference power due to signals transmitted from adjacent cells is reduced because of the low cross correlation of their spreading sequences with that of the desired user, CDMA systems can use the same carrier frequency in all the cells. This universal frequency reuse enables a mobile receiver to utilize soft handoff between cells. In other words, a mobile that is close to a cell boundary can receive the desired signal from several base stations whose signal strengths are within a certain threshold of each other. The mobile can combine the desired signal received from these base stations coherently (similar to diversity combining) to improve BER performance [31]. At other locations, the signal strength received from a base station may be much higher than that from other base stations by more than this threshold; consequently, the mobile can acquire synchronization with this base station with the strongest signal, and thereafter receive the desired signal from it. The base stations in a multicellular CDMA system transmit a pilot signal that is used by the mobiles for the purpose of synchronization, signal strength measurement, fading amplitude measurement, coherent demodulation and the acquisition of timing of the spreading sequences [31].

In this chapter, we investigate the BER performance on the forward links of CDMA 
systems that use the Walsh/m-sequence for bandwidth spreading in a multicell environment. We use the Gaussian approximation for evaluating the BER and compare the results obtained through this approach to those obtained by Monte Carlo simulation. First, we present the system and channel models which are modified to take into consideration path loss, shadowing and issues such as handoff. Then, we carry out the BER analysis using the Gaussian approximation. Finally we show some performance results.

\subsection{System and Channel Model}

The $q$ th cell contains $K_{q}$ mobile receivers which are uniformly located spatially in a regular hexagonal shaped cell. The base station is located at the center of the cell, and has its own unique m-sequence (or different shifted versions of the same m-sequence can be used for each base station). This m-sequence is used for concatenation with Walsh sequences to form the spreading sequences for signals on the forward links. The distance from the base station to the corner of a cell is denoted as $R$. The communication link between the base station and any mobile suffer a path loss that is proportional to $r^{p}$, where $r$ is the distance between the mobile and the base station, and $p$ is the path loss exponent. In urban macrocellular environments, $p \approx 4.0$ [32]. In conjunction with the path loss, there is also shadowing of the communication link. This shadowing is assumed to be lognormally distributed with $0 \mathrm{~dB} \log$ mean and $\log$ standard deviation of $8 \mathrm{~dB}$ [32]. The combined effect of path loss and lognormal shadowing gives a path gain given by

$$
\text { Path Gain }=r^{-p} 10^{\zeta / 10}
$$

where $\zeta \sim N(0,8)$. As in Chapters 3 and 4 , we assume that there is multipath fading superimposed on the lognormal shadowing and path loss. In macrocellular mobile channel environments, a Rayleigh distribution is commonly assumed for the multipath fading [9]. We assume that there are $L_{q}+1$ paths between the $q$ th base station and any mobile, with 
the signal on the $n$th path having an amplitude gain of $\alpha_{q n}=\gamma_{q n} A_{q n}$ and transmission delay of $\tau_{q n}$. Here, $\gamma_{q n}^{2}$ denotes the power gain of the signal received through the $n$th path from the $q$ th base station, and $A_{q n}$ is the random fading envelope which has a Rayleigh distribution with unit second moment. Also the carrier phase, $\phi_{q n}$, of the signal on the $n$th path at the mobile is independently and uniformly distributed on $[0,2 \pi)$.

With the existence of many base stations, we assume that the mobile measures the average received power from the base stations, and receives the desired signal from the base station with the lowest attenuation. The advantage of this is to combat the effect of shadowing and improve on the received SNR. Consequently, a mobile may be receiving its desired signal from a base station other than the one in the cell in which it is located. We further assume that the average power of the signals transmitted from the base stations to each user is constant at $\mathcal{P}$. This assumption is necessary to simplify analysis. Although forward power allocation (whereby the base station allocates more power to mobiles that experience excessive interference from other base stations) can improve the BER of mobiles that are located at the corners of a cell, however, it is reported in [33] that the area-averaged BER does not change much with the application of forward power allocation.

The mobile uses a Rake receiver that has $B+1$ fingers, and adaptively search out the $B+1$ desired multipath signals from the base station with the smallest path attenuation. Let the base station from which the desired signal is being received be indexed with 0 . Without loss of generality, we set the transmission delay of the signal in the zeroth path from this base station to zero, i.e. $\tau_{00}=0$. We measure all other signal transmission delays relative to $\tau_{00}$. We can also normalize the distance between the cell corner and the center of the cell to unity, i.e. $R=1.0$, and let $E_{b}=\mathcal{P} T$ be the average received bit energy at the corner of a cell. 
We now derive expressions for the area-averaged BER in a multiple cell CDMA system that use the orthogonal Walsh/m-sequence for the bandwidth spreading of signals transmitted through the forward link. In the analysis, we neglect the effect of mobiles in soft handoffs receiving the same signal from multiple base stations. Also, we only consider interference from the first two surrounding rings of base stations, since the interference from other base stations can be neglected because of their greater path loss.

\subsection{BER for Rayleigh faded channels}

The decision variable from the output of the Rake receiver can be obtained by modifying (4.2), and is given by

$$
U_{i}=\sqrt{\frac{\mathcal{P}_{0}^{\prime}}{2}} T\left\{b_{0}^{i} \sum_{n=0}^{B}\left|\alpha_{0 n}\right|^{2}+F_{m}\right\}+\sum_{q=1}^{18} \sqrt{\frac{\mathcal{P}_{q}^{\prime}}{2}} T I_{q}+n^{\prime}
$$

where $I_{q}$ is the interference due to signals received over the $L_{q}+1$ paths from the $q$ th base station. In (5.2), the effect of path loss and shadowing are accounted for by the modified average received power. Thus for the $q$ th base station, the average received power is given by

$$
\mathcal{P}_{q}^{\prime}=\mathcal{P r}_{q}^{-4} 10^{\zeta_{q} / 10}
$$

where $r_{q}$ is the distance between the reference mobile and the base station, and $\zeta_{q}$ accounts for the shadowing of the $q$ th base station. Following the same steps as in [31], we may express the shadowing component $\zeta_{q}$ as the sum of two components: one in the near field of the mobile that is common to all base stations, $\xi$, and one that is only dependent on the $q$ th base station, $\xi_{q}$. Thus the shadowing parameter $\zeta_{q}$ can be expressed as

$$
\zeta_{q}=a \xi+b \xi_{q}
$$

where $a^{2}+b^{2}=1$ [31]. In (5.2), the parameter $F_{m}$ is the multipath interference due to all the signals transmitted by the controlling base station, and is zero mean with a variance 
given in (4.21) with $K$ replaced by $K_{0}, \gamma_{n}$ replaced by $\gamma_{0 n}$ and $\alpha_{n}$ replaced by $\alpha_{0 n}$. The parameter $I_{q}$ which is the interference due to the signals from the $q$ th base station is also zero mean with a variance given by [4]

$$
\operatorname{var}\left(I_{q}\right)=\frac{K_{q}}{3 M} \sum_{n=0}^{B}\left|\alpha_{0 n}\right|^{2} \sum_{n=0}^{L_{q}} \gamma_{q n}^{2} .
$$

In (5.2), $n^{\prime}$ which is due to AWGN is a zero mean Gaussian random variable with a variance given by [4]

$$
\operatorname{var}\left(n^{\prime}\right)=\frac{N_{0} T}{4} \sum_{n=0}^{B}\left|\alpha_{0 n}\right|^{2} .
$$

From (5.2), the conditional SNR at the output of the Rake receiver for a mobile at a distance of $r_{0}$ from the controlling base station is given by

$$
\operatorname{SNR}\left(\alpha_{00}, \cdots, \alpha_{0 B}\right)=\frac{\frac{\mathcal{P}_{0}^{\prime} T^{2}}{2}\left(\sum_{n=0}^{B}\left|\alpha_{0 n}\right|^{2}\right)^{2}}{\operatorname{var}\left(n^{\prime}\right)+\frac{\mathcal{P}_{0}^{\prime} T^{2}}{2} \operatorname{var}\left(F_{m}\right)+\sum_{q=1}^{18} \frac{\mathcal{P}_{q}^{\prime} T^{2}}{2} \operatorname{var}\left(I_{q}\right)} .
$$

Using (5.3), the conditional SNR can be expressed as

$$
\operatorname{SNR}\left(\alpha_{00}, \cdots, \alpha_{0 B}\right)=\frac{r_{0}^{-4} 10^{\zeta_{0} / 10}\left(\sum_{n=0}^{B}\left|\alpha_{0 n}\right|^{2}\right)^{2}}{\frac{N_{0}}{2 E_{b}} \sum_{n=0}^{B}\left|\alpha_{0 n}\right|^{2}+r_{0}^{-4} 10^{\zeta_{0} / 10} \operatorname{var}\left(F_{m}\right)+\sum_{q=1}^{18} r_{q}^{-4} 10^{\zeta_{q} / 10} \operatorname{var}\left(I_{q}\right)} .
$$

The conditional SNR can be simplified further by noting that since the environment is an interference limited one, we can neglect the background noise. Also, if the links between the mobile and the base stations have identical MIP's i.e. $\gamma_{q n}=\gamma_{n}$, and the base stations transmit to an equal number, $K$, of mobiles, the variance of the interference due to the signals from any base station other than the controlling one is given by

$$
\operatorname{var}\left(I_{q}\right)=\operatorname{var}(I)=\frac{K}{3 M} \sum_{n=0}^{B}\left|\alpha_{0 n}\right|^{2} \sum_{n=0}^{L} \gamma_{n}^{2} .
$$


Consequently, the conditional SNR becomes

$$
\operatorname{SNR}\left(\alpha_{00}, \cdots, \alpha_{0 B}\right)=\frac{\left(\sum_{n=0}^{B}\left|\alpha_{0 n}\right|^{2}\right)^{2}}{\operatorname{var}\left(F_{m}\right)+y_{r_{0}} \operatorname{var}(I)}
$$

where $y_{r_{0}}$ is the ratio of the sum of the average received power from the other base stations to the average received power from the controlling base station. Since the mobile will decide on the controlling base station based on its distance and the shadowing of the signals from the different base station, we can express $y_{r_{0}}$ as

$$
\begin{aligned}
y_{r_{0}} & =\frac{\sum_{\substack{l=1 \\
l \neq n}}^{19} r_{l}^{-4} 10^{b \xi_{l} / 10}}{r_{0}^{-4} 10^{b \xi_{0} / 10}} \quad \text { given that } \\
r_{0}^{-4} 10^{b \xi_{0} / 10} & =\max _{j=1, \cdots, 19}\left\{r_{j}^{-4} 10^{b \xi_{j} / 10}\right\} .
\end{aligned}
$$

Using the Gaussian approximation, for BPSK modulation, the conditional BER is given by

$$
P_{e}\left(\alpha_{00}, \cdots, \alpha_{0 B}\right)=\mathrm{Q}\left(\sqrt{\operatorname{SNR}\left(\alpha_{00}, \cdots, \alpha_{0 B}\right)}\right)
$$

The BER at location specified by $\left(r_{1}, r_{2}, \cdots, r_{19}\right)$ can be obtained by averaging $(5.12)$ over the amplitude gain parameters $\alpha_{00}, \cdots, \alpha_{0 B}$. If we know the area-averaged distribution of $y_{r_{0}}$, i.e. averaging over the entire cell, we can obtain the area-averaged BER by using this distribution. Let the area-averaged value of $y_{r_{0}}$ be given by $y$. We can approximate the distribution of $y$ by a lognormal distribution. The reason for this approximation is that the sum of independent lognormal random variables is well approximated by another lognormal random variable [34]. In the next section, we obtain the first and second moments of $y$, and compare a lognormal fit with these moments to a simulated pdf of $y$. 


\subsection{First and Second Moments of $y$}

To get the moments of $y$, we make the simplifying assumption that the mobile is far more likely to be controlled by the base station in the cell in which the mobile is located or one of the base stations in the first tier of six surrounding cells. Let the index of the base station in which the mobile is located be 1 , and those of the first tier of surrounding base stations be $2, \cdots, 7$. The first moment of $y$ is given by

$$
\begin{array}{r}
E[y]=\iint_{\mathrm{A}_{c}}\left[\sum _ { n = 1 } ^ { 7 } \left(\sum _ { \substack { j = 1 \\
j \neq n } } ^ { 1 9 } ( \frac { r _ { n } } { r _ { j } } ) ^ { 4 } E \left[10^{b\left(\xi_{n}-\xi_{j}\right) / 10}\right.\right.\right. \\
\left.\left.\left.r_{n}^{4} 10^{b \xi_{n} / 10} \leq r_{j}^{4} 10^{b \xi_{j} / 10}, j=1, \cdots, 7\right]\right)\right] \frac{d \mathrm{~A}}{\mathrm{~A}_{c}}
\end{array}
$$

where the indices $8, \cdots, 19$ are used for base stations in the second tier, $A_{c}$ is the area of the cell and $d \mathrm{~A}$ is the variable of integration over the area of the cell. Letting $\rho=$ $\ln (10) / 10$, and noting that $x_{j n}=\xi_{n}-\xi_{j}$ is a zero mean Gaussian random variable with variance $2 \sigma^{2}$, where $\sigma^{2}=\operatorname{var}\left(\xi_{j}\right)$ for $j=1, \cdots, 19$, we can express (5.13) as

$$
\begin{array}{r}
E[y]=\iint_{\mathrm{A}_{c}}\left[\sum _ { n = 1 } ^ { 7 } \left(\sum _ { \substack { j = 1 \\
j \neq n } } ^ { 1 9 } ( \frac { r _ { n } } { r _ { j } } ) ^ { 4 } E \left[e^{b \rho x_{j n}} \mid\right.\right.\right. \\
\left.\left.\left.\xi_{j} \geq \xi_{n}+\left(M_{n}-M_{j}\right) / b, j=1, \cdots, 7\right]\right)\right] \frac{d \mathrm{~A}}{\mathrm{~A}_{c}}
\end{array}
$$

where $M_{n}=40 \log \left(r_{n}\right)$. Since $\xi_{j}$ is independent of $\xi_{k}$, the first moment of $y$ is further simplified to [31]

$$
\begin{array}{r}
E[y]=e^{(b \rho \sigma)^{2}} \iint_{\mathrm{A}_{c}}\left[\sum _ { n = 1 } ^ { 7 } \left(\sum _ { \substack { j = 1 \\
j \neq n } } ^ { 1 9 } ( \frac { r _ { n } } { r _ { j } } ) ^ { 4 } \left[\int_{-\infty}^{\infty} \frac{e^{-\left(\xi_{n}-b \rho \sigma^{2}\right)^{2} / 2 \sigma^{2}}}{\sqrt{2 \pi} \sigma} d \dot{\xi}_{n}\right.\right.\right. \\
\int_{\xi_{n}+\left(M_{n}-M_{j}\right) / b}^{\infty} \frac{e^{-\left(\xi_{j}+b \rho \sigma^{2}\right)^{2} / 2 \sigma^{2}}}{\sqrt{2 \pi} \sigma} d \xi_{j} \\
\left.\left.\left.\prod_{\substack{w=1 \\
w \neq n \\
w \neq j}}^{7} \int_{\xi_{n}+\left(M_{n}-M_{w}\right) / b}^{\infty} \frac{e^{-\xi_{w}^{2} / 2 \sigma^{2}}}{\sqrt{2 \pi} \sigma} d \xi_{w}\right]\right)\right] \cdot \frac{d \mathrm{~A}}{\mathrm{~A}_{c}}
\end{array}
$$


where we have re-indexed the variables associated with the 7 most probable controlling base stations with $w$. Following the same steps as in [31] and changing variables, (5.15) can be simplified to

$$
\begin{array}{r}
E[y]=e^{(b \rho \sigma)^{2}} \iint_{\mathrm{A}_{c}}\left[\sum _ { n = 1 } ^ { 7 } \left(\sum _ { \substack { j = 1 \\
j \neq n } } ^ { 1 9 } ( \frac { r _ { n } } { r _ { j } } ) ^ { 4 } \left[\int_{-\infty}^{\infty} \frac{e^{-\left(z^{2}\right)^{2} / 2}}{\sqrt{2 \pi}}\right.\right.\right. \\
\left.\left.\left.\mathrm{Q}\left(z+\frac{M_{n}-M_{j}}{b \sigma}+2 b \rho \sigma\right) \prod_{\substack{w=1 \\
w \neq n \\
w \neq j}}^{7} \mathrm{Q}\left(z+\frac{M_{n}-M_{w}}{b \sigma}+b \rho \sigma\right)\right]\right)\right] \frac{d \mathrm{~A}}{\mathrm{~A}_{c}}
\end{array}
$$

The mean of $y$ was evaluated numerically over the area of the cell. Using $b=1 / \sqrt{2}[31]$ and $\sigma=8[32]$, we found that $E[y] \approx 0.51$.

Following the same procedure as for the first moment of $y$, the second moment of $y$ can be written as

$$
\begin{gathered}
E\left[y^{2}\right]=\iint_{\mathrm{A}_{c}}\left[\sum _ { n = 1 } ^ { 7 } E \left[\left(\sum_{\substack{j=1 \\
j \neq n}}^{19}\left(\frac{r_{n}}{r_{j}}\right)^{4} 10^{b x_{j n} / 10}\right)^{2} \mid\right.\right. \\
\left.\left.r_{n}^{4} 10^{b \xi_{n} / 10} \leq r_{j}^{4} 10^{b \xi_{j} / 10}, j=1, \cdots, 7\right]\right] \frac{d \mathrm{~A}}{\mathrm{~A}_{c}} .
\end{gathered}
$$

By expanding the square, the second moment can be written as

$$
\begin{array}{r}
E\left[y^{2}\right]=\iint_{\mathrm{A}_{c}}\left[\sum _ { n = 1 } ^ { 7 } \sum _ { \substack { j = 1 \\
j \neq n } } ^ { 1 9 } ( \frac { r _ { n } } { r _ { j } } ) ^ { 8 } E \left[10^{2 b x_{j n} / 10} \mid r_{n}^{4} 10^{b \xi_{n} / 10} \leq r_{j}^{4} 10^{b \xi_{j} / 10}\right.\right. \\
j=1, \cdots, 7]+2 \sum_{\substack{k=1 \\
k \neq n}}^{18} \sum_{\substack{l=k+1 \\
l \neq n}}^{19}\left(\frac{r_{n}^{2}}{r_{l} r_{k}}\right)^{4} E\left[10^{b\left(x_{n k}-x_{n l}\right) / 10} \mid\right. \\
\left.\left.r_{n}^{4} 10^{b \xi_{n} / 10} \leq r_{j}^{4} 10^{b \xi_{j} / 10}, j=1, \cdots, 7\right]\right] \frac{d \mathrm{~A}}{\mathrm{~A}_{c}} .
\end{array}
$$

Following the same procedure as before, we can simplify the second moment of $y$ to

$$
\begin{gathered}
E\left[y^{2}\right]=\iint_{\mathrm{A}_{c}}\left[\sum _ { n = 1 } ^ { 7 } \sum _ { \substack { j = 1 \\
j \neq n } } ^ { 1 9 } ( \frac { r _ { n } } { r _ { j } } ) ^ { 8 } e ^ { ( 2 b \rho \sigma ) ^ { 2 } } \left[\int_{-\infty}^{\infty} \frac{e^{-\left(\xi_{n}-2 b \rho \sigma^{2}\right)^{2} / 2 \sigma^{2}}}{\sqrt{2 \pi} \sigma} d \xi_{n}\right.\right. \\
\left.\int_{\xi_{n}+\left(M_{n}-M_{j}\right) / b}^{\infty} \frac{e^{-\left(\xi_{j}+2 b \rho \sigma^{2}\right)^{2} / 2 \sigma^{2}}}{\sqrt{2 \pi} \sigma} d \xi_{j} \prod_{\substack{w=1 \\
\text { wan } \\
w \neq j}}^{7} \int_{\xi_{n}+\left(M_{n}-M_{w}\right) / b}^{\infty} \frac{e^{-\xi_{w}^{2} / 2 \sigma^{2}}}{\sqrt{2 \pi} \sigma} d \xi_{w}\right]
\end{gathered}
$$




$$
\begin{array}{r}
+2 \sum_{\substack{k=1 \\
k \neq n}}^{18} \sum_{\substack{l=k+1 \\
l \neq n \\
l \neq n}}^{19}\left(\frac{r_{n}^{2}}{r_{l} r_{k}}\right)^{4} e^{3(b \rho \sigma)^{2}}\left[\int_{-\infty}^{\infty} \frac{e^{-\left(\xi_{n}-2 b \rho \sigma^{2}\right)^{2} / 2 \sigma^{2}}}{\sqrt{2 \pi} \sigma} d \xi_{n}\right. \\
\int_{\xi_{n}+\left(M_{n}-M_{k}\right) / b}^{\infty} \frac{e^{-\left(\xi_{k}+b \rho \sigma^{2}\right)^{2} / 2 \sigma^{2}}}{\sqrt{2 \pi} \sigma} d \xi_{k} \int_{\xi_{n}+\left(M_{n}-M_{l}\right) / b}^{\infty} \frac{e^{-\left(\xi_{l}+b \rho \sigma^{2}\right)^{2} / 2 \sigma^{2}}}{\sqrt{2 \pi} \sigma} d \xi_{l} \\
\left.\left.\prod_{\substack{w=1 \\
w \neq n \\
w \neq k \\
w \neq l}}^{7} \int_{\xi_{n}+\left(M_{n}-M_{w}\right) / b}^{\infty} \frac{e^{-\xi_{w}^{2} / 2 \sigma^{2}}}{\sqrt{2 \pi} \sigma} d \xi_{w}\right]\right] \frac{d \mathrm{~A}}{\mathrm{~A}_{c}}
\end{array}
$$

Following the same steps as in [31] and changing variables, (5.19) can be simplified to

$$
\begin{array}{r}
E\left[y^{2}\right]=e^{(2 b \rho \sigma)^{2}} \iint_{\mathrm{A}_{c}}\left[\sum _ { n = 1 } ^ { 7 } \sum _ { \substack { j = 1 \\
j \neq n } } ^ { 1 9 } ( \frac { r _ { n } } { r _ { j } } ) ^ { 8 } \left[\int_{-\infty}^{\infty} \frac{e^{-z^{2} / 2}}{\sqrt{2 \pi}}\right.\right. \\
\left.\mathrm{Q}\left(z+\frac{M_{n}-M_{j}}{b \sigma}+4 b \rho \sigma\right) \prod_{\substack{w=1 . \\
w \neq n \\
w \neq j}}^{7} \mathrm{Q}\left(z+\frac{M_{n}-M_{w}}{b \sigma}+2 b \rho \sigma\right) d z\right] \\
+2 \sum_{\substack{k=1 \\
k \neq n}}^{18} \sum_{\substack{l=k+1 \\
l \neq n}}^{19}\left(\frac{r_{n}^{2}}{r_{l} r_{k}}\right)^{4} e^{-(b \rho \sigma)^{2}}\left[\int_{-\infty}^{\infty} \frac{e^{-z^{2} / 2}}{\sqrt{2 \pi}}\right. \\
\mathrm{Q}\left(z+\frac{M_{n}-M_{k}}{b \sigma}+3 b \rho \sigma\right) \mathrm{Q}\left(z+\frac{M_{n}-M_{l}}{b \sigma}+3 b \rho \sigma\right) \\
\left.\prod_{\substack{w=1 \\
w \neq n \\
w \neq k \\
w \neq l}}^{7} \mathrm{Q}\left(z+\frac{M_{n}-M_{w}}{b \sigma}+2 b \rho \sigma\right) d z\right] \frac{d \mathrm{~A}}{\mathrm{~A}_{c}} .
\end{array}
$$

Using $b=1 / \sqrt{2}$ and $\sigma=8$, the second moment of $y$ was evaluated numerically to give $E\left[y^{2}\right] \approx 0.625$. The pdf of $y$ is plotted with these parameters using the lognormal distribution and is compared to the simulated pdf of $y$ in Figure 5.1(a). The method to simulate the pdf of $y$ is fully explained in $[31,35]$.

There is a reasonable agreement between the theoretical pdf and the simulated one for small values of $y$. A possible reason for the deviation of the theoretical pdf from the simulated one is that the lognormal random variables that are being added are dependent. The effect of the mobile selecting the base station with the smallest attenuation tends to reduce the probability of high values of $y$ as seen in the simulated pdf of $y$. Shown in 
(a) Controlling base station IS The one With LoWest attenuation

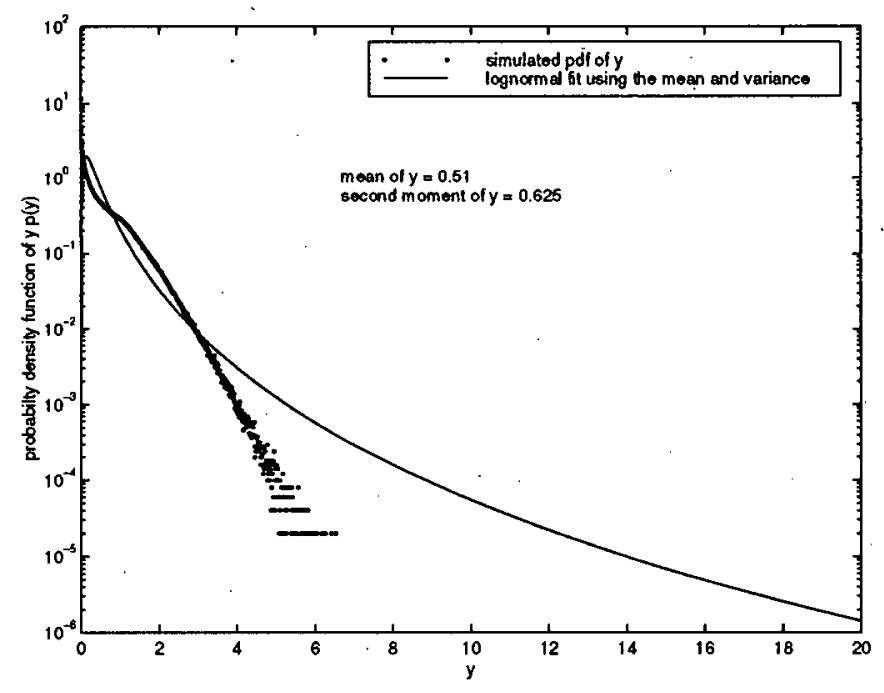

(b) Controlling Base station IS AlWAys the one located in THE SAME CELL AS THE MOBILE

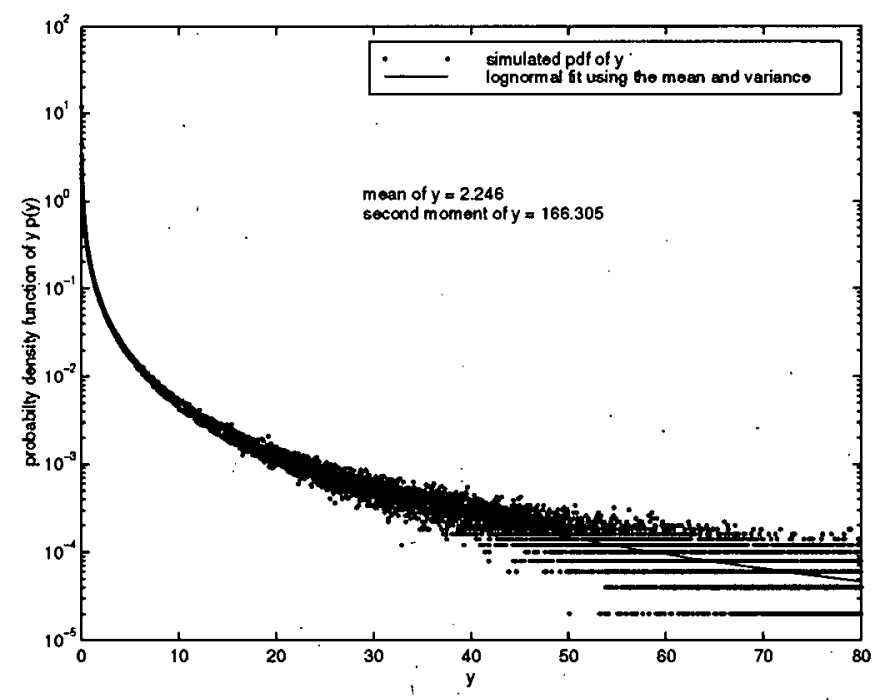

Figure 5.1: Probability density function of the the ratio of the sum of the average received power from the other base stations to the average received power from the controlling base station 
Figure 5.1(b) is a lognormal fit to $y$, when the controlling base station is restricted to the one in the same cell as the mobile. We can see a closer agreement between the fit and the simulated pdf. This is an indication that the difference between the theoretical fit and the simulated pdf is due to the dependence between the random variables involved. From the simulations, we can also see that soft handoff (where the mobile acquires synchronization with the base station with the smallest attenuation) reduces the mean of $y$ from 2.246 to 0.51 .

\subsection{BER for Rician faded channels}

With the proposal that future urban mobile systems be configured with many small cells [36], it is useful to evaluate the BER performance on the forward link of systems using the concatenated Walsh/m-sequence for bandwidth spreading in these environments. The communication link between a mobile and a controlling base station in such urban microcellular radio systems is characterized with Rician fading [36]. Also, when the reference mobile is located in the vicinity of the controlling base station in an urban macrocellular system, the communication link may consist of a specular signal. In this section, we derive expressions for the BER on the forward communication link that use the concatenated Walsh/m-sequence for bandwidth spreading using the Gaussian approximation. This will be done for two cases: (1) the communication links between the mobile and all the base stations have a specular signal on the zeroth path, and (2) only the communication link between the mobile and the controlling base station has a specular signal on the zeroth path, while the communication links to all the other base stations have Rayleigh fading on all the paths.

For the first case, if the power gain of the specular signal between the $q$ th base station and the mobile is $\beta_{q}^{2}$, the decision variable from the output of the Rake receiver is obtained 
by modifying $(5.2)$ to

$$
U_{i}=\sqrt{\frac{\mathcal{P}_{0}^{\prime}}{2}} T\left\{b_{0}^{i}\left(\beta_{0}^{2}+\sum_{n=1}^{B}\left|\alpha_{0 n}\right|^{2}\right)+F_{m}+I_{m}\right\}+\sum_{q=1}^{18} \sqrt{\frac{\mathcal{P}_{q}^{\prime}}{2}} T I_{q}+n^{\prime}
$$

where the additional multiuser interference term $I_{m}$ is zero mean with a variance of $\operatorname{var}\left(I_{m}\right)=\gamma_{00}^{2} / 2$. Here, the multipath interference $F_{m}$ is zero mean with a variance given by (4.30) with $\beta$ replaced by $\beta_{0}, K$ replaced by $K_{0}, \gamma_{n}$ replaced by $\gamma_{0 n}$ and $\alpha_{n}$ replaced by $\alpha_{0 n}$. Also, the variance of the interference due to the signals from the $q$ th base station is modified to

$$
\operatorname{var}\left(I_{q}\right)=\frac{K_{q}}{3 M}\left(\beta_{0}^{2}+\sum_{n=1}^{B}\left|\alpha_{0 n}\right|^{2}\right)\left(\beta_{q}^{2}+\sum_{n=0}^{L_{q}} \gamma_{q n}^{2}\right)
$$

The parameter due to.AWGN is also modified to

$$
\operatorname{var}\left(n^{\prime}\right)=\frac{N_{0} T}{4}\left(\beta_{0}^{2}+\sum_{n=1}^{B}\left|\alpha_{0 n}\right|^{2}\right)
$$

The conditional SNR at the output of the Rake receiver for a mobile at a distance of $r_{0}$ from the controlling base station is consequently given by

$$
\operatorname{SNR}\left(\alpha_{01}, \cdots \alpha_{0 B}\right)=\frac{\frac{\mathcal{P}_{0}^{\prime} T^{2}}{2}\left(\beta_{0}^{2}+\sum_{n=1}^{B}\left|\alpha_{0 n}\right|^{2}\right)^{2}}{\operatorname{var}\left(n^{\prime}\right)+\frac{\mathcal{P}_{0}^{\prime} T^{2}}{2} \operatorname{var}\left(F_{m}\right)+\sum_{q=1}^{18} \frac{\mathcal{P}_{q}^{\prime} T^{2}}{2} \operatorname{var}\left(I_{q}\right)}
$$

Using the same assumptions as in Section 5.3, i.e. that the background noise power can be neglected, for identical link parameters between the base stations and the mobile (i.e. $\beta_{q}=\beta$ and $\gamma_{q n}=\gamma_{n}$ ) and an equal number of controlled mobiles per base station, the conditional SNR can be simplified to

$$
\operatorname{SNR}\left(\alpha_{01}, \cdots, \alpha_{0 B}\right)=\frac{\left(\beta^{2}+\sum_{n=1}^{B}\left|\alpha_{0 n}\right|^{2}\right)^{2}}{\operatorname{var}\left(F_{m}\right)+\operatorname{var}\left(I_{m}\right)+y_{r_{0}} \operatorname{var}(I)}
$$

where

$$
\operatorname{var}\left(I_{q}\right)=\operatorname{var}(I)=\frac{K}{3 M}\left(\beta^{2}+\sum_{n=1}^{B}\left|\alpha_{0 n}\right|^{2}\right)\left(\beta^{2}+\sum_{n=0}^{L_{q}} \gamma_{n}^{2}\right)
$$


and $y_{r_{0}}$ is the same as in (5.11).

Using the Gaussian approximation, for BPSK modulation, the conditional BER is given by

$$
P_{e}\left(\alpha_{01}, \cdots, \alpha_{0 B}\right)=\mathrm{Q}\left(\sqrt{\operatorname{SNR}\left(\alpha_{01}, \cdots, \alpha_{0 B}\right)}\right)
$$

The BER at location $r_{0}$ can be obtained by averaging (5.27) over the fading parameters $\alpha_{01}, \cdots, \alpha_{0 B}$. The area-averaged BER can be obtained by using the distribution of $y$ when $y_{r_{0}}$ is replaced by $y$.

For the second case where only the controlling base station has a specular signal in the zeroth path, the conditional SNR is also given by (5.25) with $\operatorname{var}(I)$ modified to

$$
\operatorname{var}(I)=\frac{K}{3 M}\left(\beta^{2}+\sum_{n=1}^{B}\left|\alpha_{0 n}\right|^{2}\right)\left(\sum_{n=1}^{L_{q}} \gamma_{n}^{2}+\gamma_{0}^{\prime 2}\right)
$$

where $\gamma_{0}^{\prime 2}$ represent the average power gain in the faded signal on the zeroth path between a non controlling base station and the mobile. The area-averaged BER can similarly be obtained by averaging (5.27) over the amplitude gain parameters $\alpha_{01}, \cdots, \alpha_{0 B}$ and $y$. It should be noted from (5.26) and (5.28) that when $\gamma_{0}^{\prime 2}=\beta^{2}+\gamma_{0}^{2}$, the Gaussian approximation will give the same area-averaged BER in both cases.

\subsection{Numerical Results}

We follow the same approach as in Chapters 3 and 4 in presenting numerical results for the forward link of the system in a multicell environment, i.e. weak and strong multipaths signals in both Rayleigh and Rician channel environments. In the simulation results presented, the same procedure of simulating the average BER as in Chapter 4 was used. Different m-sequences of period 2047 selected from the set of sequences in [37] were used for each of the 19 base stations. Their characteristic polynomials represented in octal notation are $5001,4027,4053,4055,4107,4143,4145,4767,4005,5007,5023,5025,5051,5111$, 
$5141,5155,5253,5263$ and 5337 . We assume no timing synchronization of the sequences between the different base stations, thus, BER results are averages over all possible timing offsets between the different base stations. It should be noted that for each bit during the simulation, a random mobile location (in the center cell) is used, and independent lognormal shadowing is used for each base station. The controlling base station is selected from the set of 19 base stations as the one with the lowest attenuation due to its distance and shadowing from the mobile.

Figures 5.2 and 5.3 show the simulated area-averaged BER for a system with Rayleigh fading on the zeroth path. Both figures also show the area-averaged BER obtained by an averaging using the approximate lognormal distribution of $y$ and that obtained by using the mean of $y$ in the Gaussian approximation expression (5.12). Figure 5.2 shows that averaging with the approximate distribution of $y$ gives a BER closer to that obtained from Monte Carlo simulation than that obtained by using the mean of $y$. It should be noted that in [4] the mean of $y \cong 0.77$ (for a situation where the mobile is controlled by the base station with the smallest attenuation from the two closest ones) is used. The BER that results from selecting the controlling base station from just the two closest ones is higher compared to that when this selection is done from the larger set of seven base stations. Figure 5.3 which shows the BER for a channel environment with strong multipaths also indicates that the BER obtained by using the mean of $y$ is more optimistic compared to that obtained by averaging with the distribution of $y$ for $K \leq 35$.

Figures 5.4 and 5.5 show the simulated area-averaged BER for a system with Rician fading on the zeroth path and the area-averaged BER obtained by an averaging using the approximate lognormal distribution of $y$ and that obtained by using the mean of $y$ in the Gaussian approximation expression (5.27). In both figures, $\beta=1.0$ and $\gamma_{0}=0$. In Figure 5.4, for a channel with weak multipath signals, BER curves are shown for (1) an environment where the communication link between all the base stations and the mobile 
contains a specular signal of amplitude $\beta$, and for (2) an environment where only the controlling base station has a specular signal of amplitude $\beta$. In the second environment, $\gamma_{0}^{\prime}=1.0$. It can be seen that the BER obtained from these two environments are practically the same. We can also observe that the BER obtained using the mean of $y$ is more optimistic compared to that from using the approximate distribution of $y$ in the Gaussian approximation expression (5.27). Figure 5.5 which shows the BER for a channel environment with strong multipath signals also reveals the same trend for $K \leq 45$. 


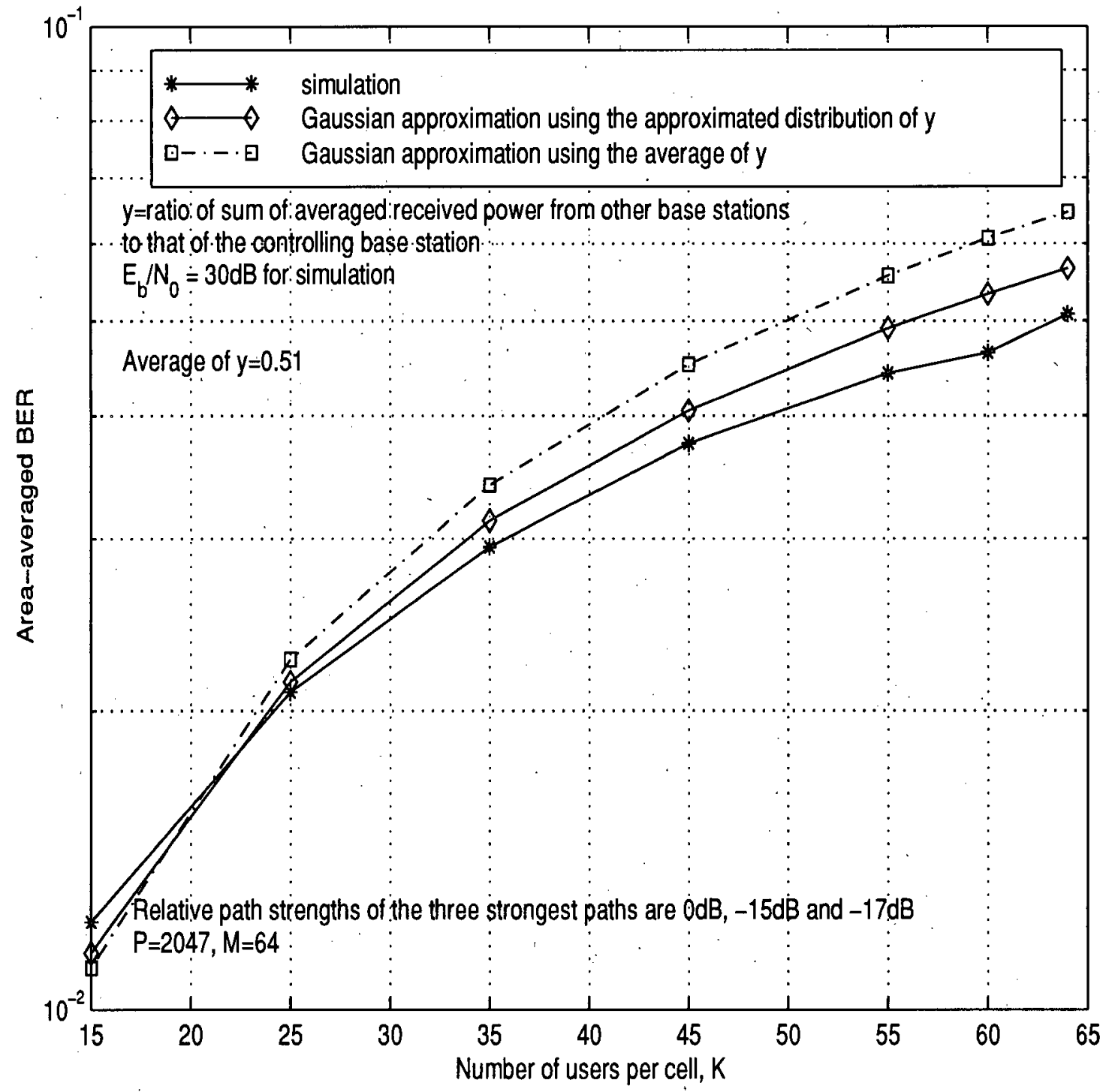

Figure 5.2: Area-averaged BER in a Rayleigh faded channel with weak multipaths, $M=64, P=2047$ (Rake receiver) 


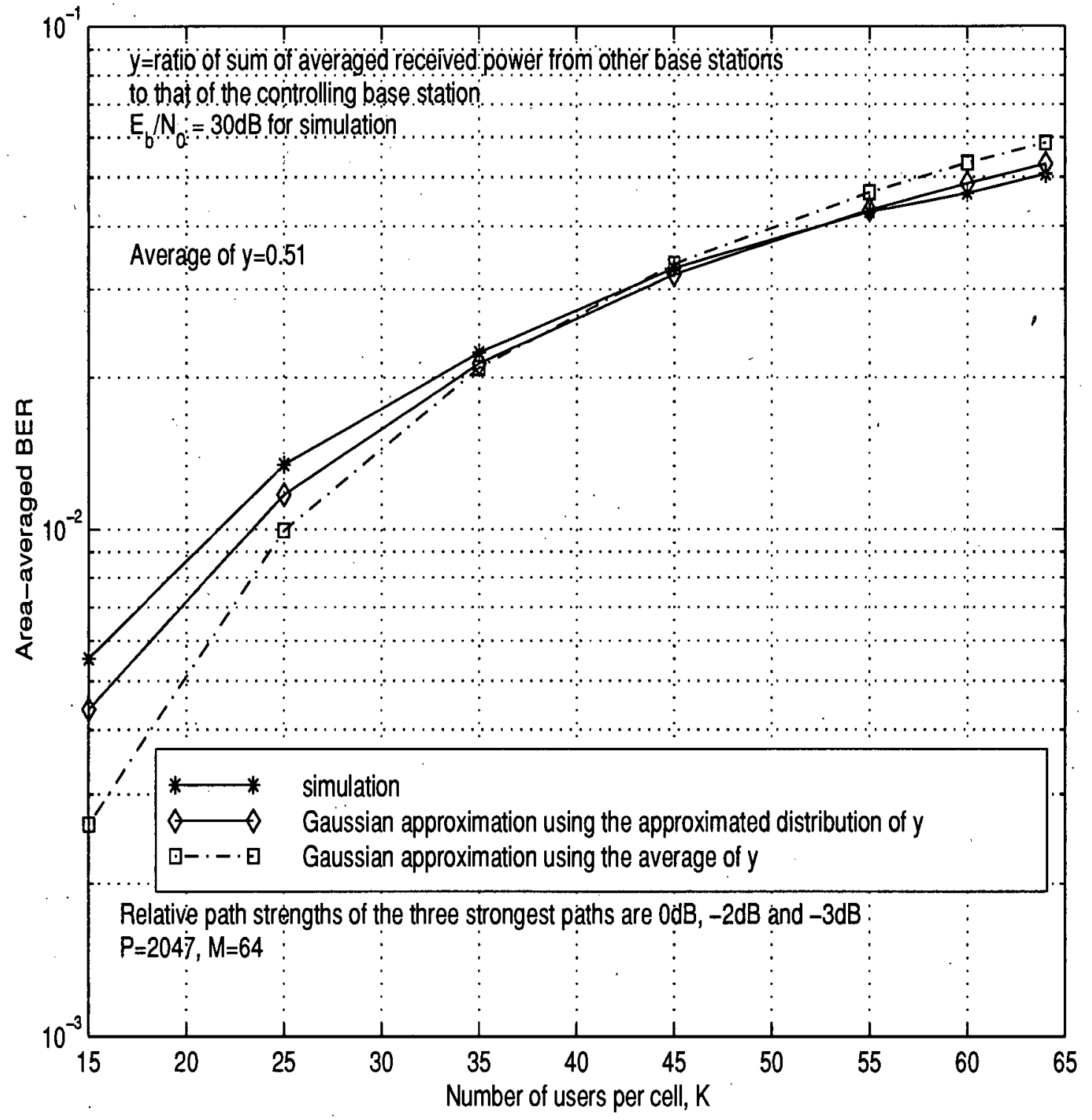

Figure 5.3: Area-averaged BER in a Rayleigh faded channel with strong multipaths, $M=64, P=2047$ (Rake receiver) 


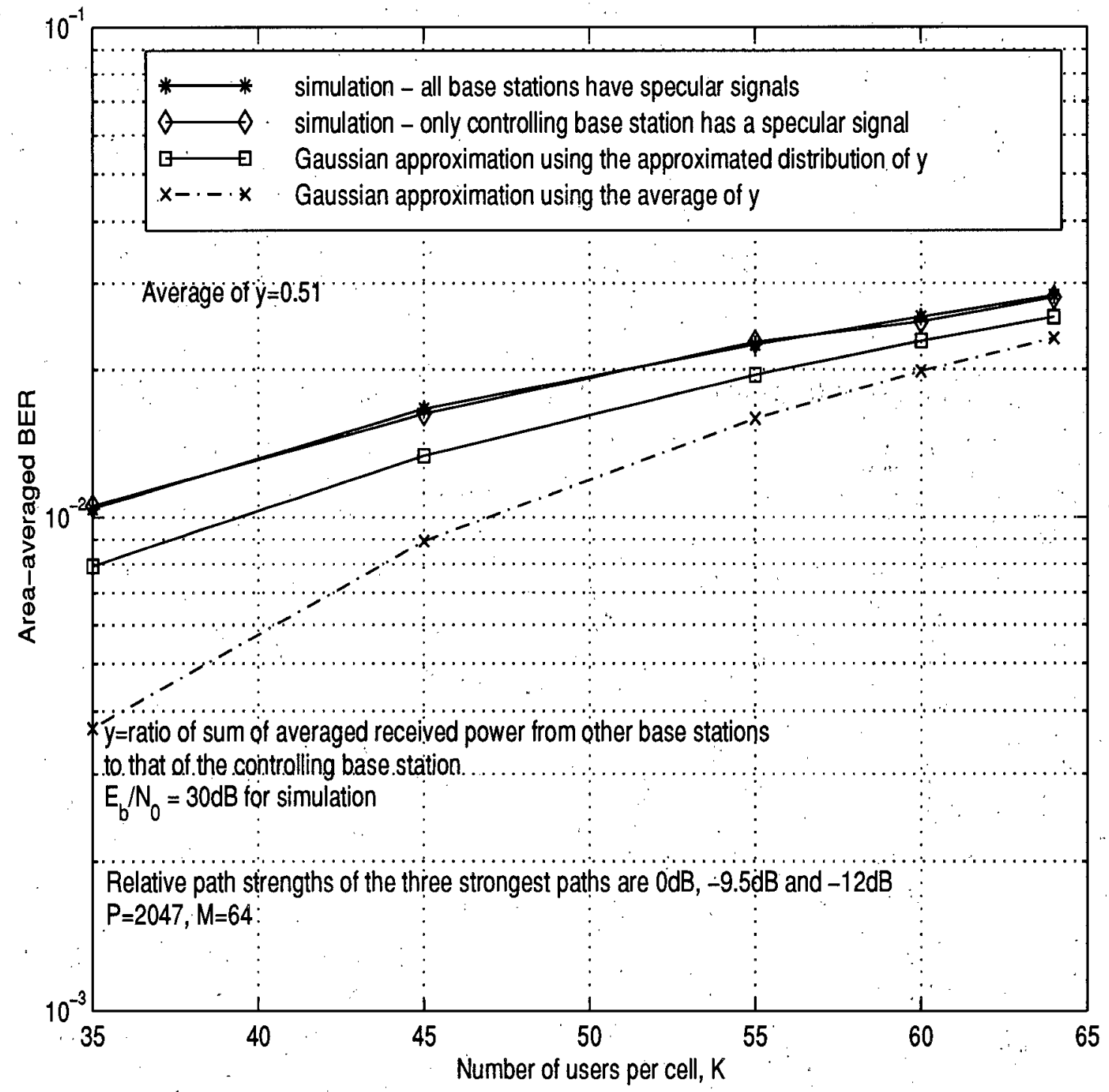

Figure 5.4: Area-averaged BER in a Rician faded channel with weak multipaths, $M=64, P=2047$ (Rake receiver) 


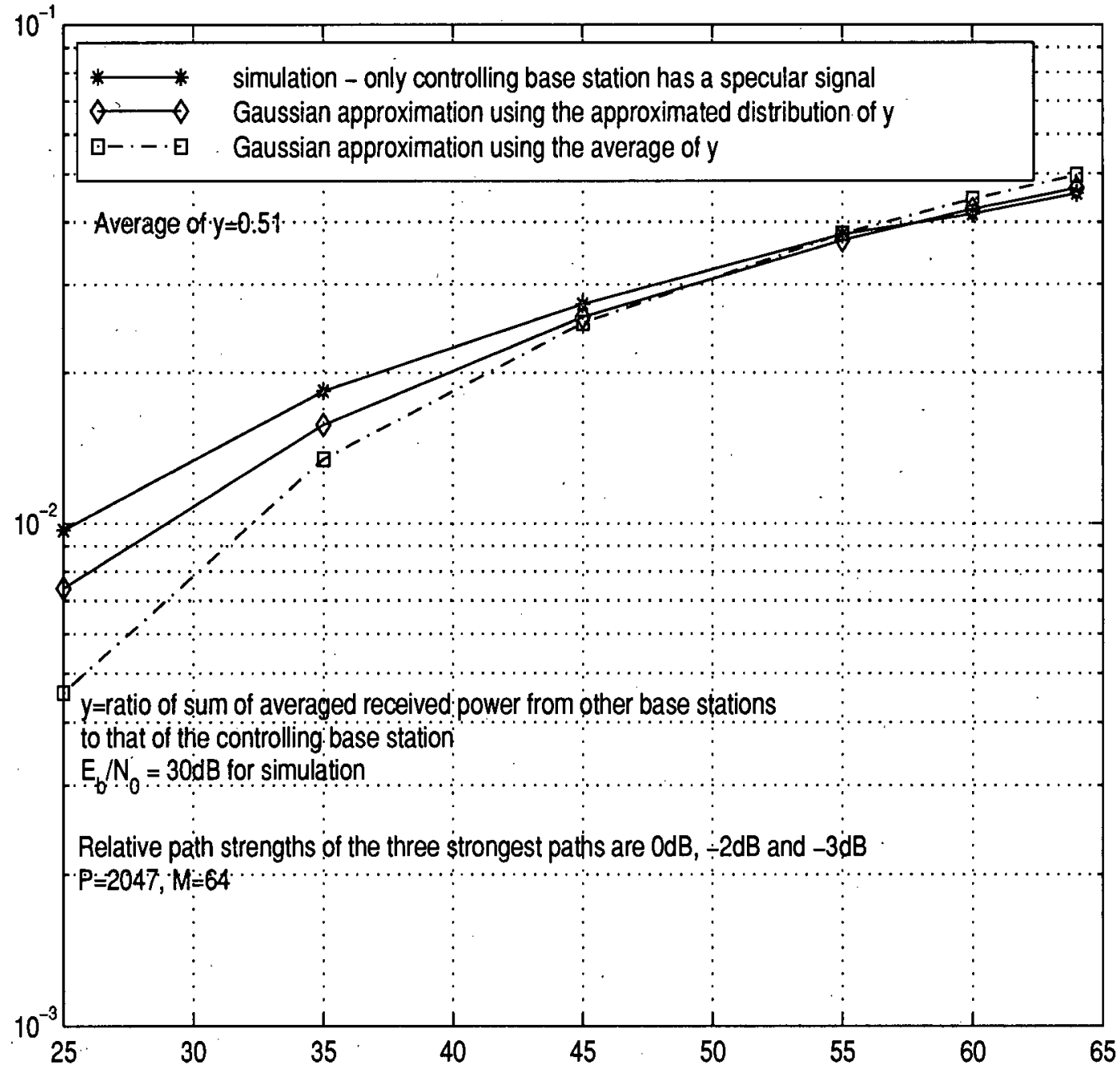

Figure 5.5: Area-averaged BER in a Rician faded channel with strong multipaths, $M=64, P=2047$ (Rake receiver) 


\section{Chapter 6}

\section{Conclusion and Further Study}

\subsection{Introduction}

In this thesis, the BER performance on the forward links of CDMA systems using a concatenated orthogonal Walsh/m-sequence scheme $[3,4]$ for bandwidth spreading has been studied. In this chapter, we summarize the main contributions of this thesis and provide some suggestions for further study.

\subsection{Contributions of the Thesis and Suggestions for Further Study}

\section{An expression for evaluating the BER using the characteristic function approach}

An expression is derived for evaluating the BER for the forward link of a single cell CDMA system both in a frequency selective Rayleigh and Rician fading channel environment using the characteristic function approach. Unfortunately, the computational time that is required to obtain the BER using this expression increases rapidly (as $P \times K \times 4^{K}$ ) with the number of users, $K$, in the system. Since the characteristic function approach is exact, it would be useful to study ways of reducing computation complexity of this approach. To this end, the behaviour of the concatenated sequences for long period m-sequences which is similar to that of random sequences can be exploited. 


\section{The effect of the period of the m-sequence}

It is observed that when a short period m-sequence is used in concatenating with Walsh sequences to form the spreading code, a somewhat lower average BER is obtained, for a small number of users, compared to when a long period m-sequence is used. The BER performance, however, is approximately the same for systems using short or long period $\mathrm{m}$-sequences as the number of users increase. It is also observed that for short period msequences and for a small number of users, the BER performance can vary much with the set of Walsh sequences that are allocated to the users. A future study may be required to select the set of Walsh sequences that give the best performance for a particular number of users.

\section{Accuracy of the Gaussian Approximation}

Another contribution of the thesis is the observation that the Gaussian approximation is very inaccurate in estimating the BER for the forward link of a CDMA system using the concatenated Walsh/m-sequence for bandwidth spreading. This inaccuracy is especially large in a single cell system in a frequency selective Rician fading environment where the mobile uses a correlation receiver. Also, for single cell systems, BER estimates by the Gaussian approximation in both Rayleigh and Rician fading environments are very optimistic when Rake receivers are used. The explanation for this is that the Gaussian approximation, which is based on the central limit theorem, is not really applicable for the forward link since the interference terms due to the simultaneously transmitted signals by the base station to other users are not statistically independent. The carrier phases, the transmission delays of the multipaths and the fading amplitudes of the simultaneously transmitted signals are the same at the mobile. 


\section{A modified Rake receiver}

The further contribution of the thesis is the observation that for channel environments where the multipath signals (other than the zeroth path signal) are weak, the maximal ratio combining Rake receiver, which is optimum in AWGN, is not optimum in the interference environment of a CDMA system using the concatenated Walsh/m-sequence for bandwidth spreading. For such a system and for Rician fading with weak multipath signals, the BER performance of the forward link using the Rake receiver degrades more rapidly with the number of users than for a system using the conventional correlation receiver. Beyond a certain number of users, the Rake receiver actually gives a higher BER than the conventional correlation receiver. An explanation for this is that since interference due to simultaneously transmitted signals on the multipaths are eliminated because of the orthogonality of the Walsh sequences, the interference signal that is in the output of a finger which is synchronized to a path is only due to the signals in the other paths. Consequently, a finger which is synchronized to a weak signal will output more interference due to the strong signals in the zeroth path. The addition of the output of such a finger to the total decision variable, despite being weighted by the path amplitude gain, will add more interference power than desired signal power. The result is a degraded BER performance.

We modified the Rake receiver to one where the output of a finger is only included in the maximal ratio combining if its instantaneous path strength is within a certain threshold of the maximum instantaneous path strengths. When this threshold is $0 \%$, the combining technique is essentially a selection diversity type. We found that a receiver using a threshold of about $50 \%$ gave better BER performance on the average compared to both the conventional correlation receiver and the Rake receiver for the channel environments considered in this thesis. Further study is required to provide guidelines for 
choosing the optimum threshold since this varies with number of users as well as the channel environment. The structure of the optimal receiver could also be investigated.

\section{An approximate distribution to evaluate the area-averaged BER}

A final contribution of the thesis is the use of a lognormal distribution to approximate the distribution of the ratio of the sum of the total average received power from other base stations to that received from the controlling base station. This approximation of lognormal distribution was utilized in evaluating the area-averaged BER, and was found to be more accurate than using just the mean of this distribution. 


\section{Glossary}

This section provides a list of acronyms used in this thesis.

AWGN -Additive White Gaussian Noise

BER -Bit Error Rate

BPSK -Binary Phase Shift Keying

CDMA -Code Division Multiple Access

$K$-Number of Users in the system

$M$-Period of Walsh sequences and also the processing gain

MIP -Multipath Intensity Profile

$P$-Period of m-sequence

pdf -probability density function

PN -Pseudo-Noise

RF -Radio Frequency

SNR - Signal to Noise Ratio

WAL $(n, T)$-Walsh function with $n$ zero crossing during time period $T$

WSSUS -Wide Sense Stationary Uncorrelated Scattering 


\section{Bibliography}

[1] E. Geraniotis, "Direct-sequence spread-spectrum multiple-access communications over nonselective and frequency-selective Rician fading channels," IEEE Trans. on Communications, vol. 34, pp. 756-764, Aug. 1986.

[2] D. Liu, C. L. Despins, and W. A. Krzymien, "Efficient and accurate DS-SSMA deterministic signature sequence performance evaluation over wireless fading channels," IEEE Signal Processing Workshop on Signal Processing Advances in Wireless Communications, SPAWC'97, pp. 253-256, 1997.

[3] M. H. Fong and V. K. Bhargava, "Concatenated orthogonal/PN codes for DS-CDMA systems in a multi-user and multipath fading environment," IEEE Global Communications Conference, pp. 1642-1646, 1994.

[4] M. H. Fong, V. K. Bhargava, and Q. Wang, "Concatenated orthogonal/PN spreading sequences and their application to cellular DS-CDMA systems with integrated traffic," IEEE Journal on Selected Areas in Communications, vol. 14, pp. 547-558, Apr. 1996.

[5] R. Skaug and J. F. Hjelmstad, Spread Spectrum in Communication. London: Peter Peregrinus Ltd, 1985.

[6] E. Geraniotis and B. Ghaffari, "Performance of binary and quaternary directsequence spread-spectrum multiple-access systems with random signature sequences," IEEE Trans. on Communications, vol. 39, pp. 713-724, May 1991.

[7] E. Geraniotis and M. B. Pursley, "Performance of coherent direct-sequence spreadspectrum communications over specular multipath fading channels,". IEEE Trans. on Communications, vol. 33, pp. 502-508, June 1985.

[8] G. P. Efthymoglou, V. A. Aalo, and H. Helmken, "Performance analysis of coherent DS-CDMA systems in a Nakagami fading channel with arbitrary parameters," IEEE Transactions on Vehicular Technology, pp. 289-297, May 1997.

[9] J. G. Proakis, Digital Communications. McGraw-Hill Inc, third ed., 1995.

[10] M. B. Pursley and D. V. Sarwate, "Evaluation of correlation parameters for periodic sequences," IEEE Trans, on Information Theory, pp. 508-513, July 1977. 
[11] J. L. Massey and J. J. Uhran, "Sub-baud coding," Proceedings of the Thirteenth Annual Allerton Conference on Circuit and System Theory, pp. 539-547, Oct. 1975.

[12] K. Yao, "Error probability of asynchronous spread spectrum multiple access communication systems," IEEE Trans. on Communications, vol. 25, pp. 803-809, Aug. 1977.

[13] K. Yao and R. M. Tobin, "Moment space upper and lower error bounds for digital systems with intersymbol interference," IEEE Trans. on Information Theory, vol. 13, pp. 65-75, Jan. 1976.

[14] K. Cheun, "Performance of direct-sequence spread-spectrum Rake receivers with random spreading sequences," IEEE Trans. on Communications, vol. 45, pp. 11301143, Sept. 1997.

[15] K. B. Letaief, "Efficient evaluation of the error probabilities of spread-spectrum multiple-access communications," IEEE Trans. on Communications, vol. 45, pp. 239-246, Feb. 1997.

[16] J. M. Holtzman, "A simple, accurate method to calculate spread spectrum multiple access error probabilities," IEEE Trans. on Communications, vol. 40, pp. 461-464, Mar. 1992.

[17] L. Tao and X. H. Chen, "Comparison of correlation parameters of binary codes for DS/CDMA systems," Proc. IEEE Int. Conf. on Communications, pp. 1059-1063, 1994.

[18] A. Salmasi and K. S. Gilhousen, "On the system design aspect of code division multiple access (CDMA) applied to digital cellular and personal communications networks," IEEE Vehicular Technology Conference, pp. 57-62, May 1991.

[19] Z. Liu, M. J. Karol, M. El-Zarki, and K. Y. Eng, "Demand-assignment access control for multi-code DS-CDMA wireless packet (ATM) networks," IEEE Computer and Communications Societies, pp. 713-721, 1996.

[20] H. Hashemi, "Impulse response modeling of indoor radio propagation channels," IEEE Journal on Selected Areas in Communications, vol. 11, pp. 967-978, Sept. 1993.

[21] G. Wu, A. Jalali, and P. Mermelstein, "On channel model parameters for microcellular CDMA systems," IEEE Transactions on Vehicular Technology, vol. 44, pp. 706711, Aug. 1995. 
[22] V. A. Aalo, "Performance of maximal-ratio diversity systems in a correlated Nakagami-fading environment," IEEE Trans. on Communications, vol. 43, pp. 23602369, Aug. 1995.

[23] G. L. Turin, "Introduction to spread-spectrum antimultipath techniques and their application to urban digital radio," Proc. of the IEEE, vol. 68, pp. 328-353, Mar. 1980.

[24] T. S. Rappaport, S. Y. Seidel, and K. Takamizawa, "Statistical channel impulse response models for factory and open plan building radio communication system design," IEEE Trans. on Communications, vol. 39, pp. 794-807, May 1991.

[25] E. Geraniotis, "Coherent hybrid DS-SFH spread-spectrum multiple-access communications," IEEE Journal on Selected Areas in Communications, vol. 3, pp. 695-705, May 1985.

[26] E. Geraniotis, "Noncoherent hybrid DS-SFH. spread-spectrum multiple-access communications," IEEE Trans. on Communications, vol. 34; pp. 862-872, Sept. 1986.

[27] R. Ganesh and K. Pahlavan, "Statistical modeling and computer simulation of the indoor radio channel," IEE Proc. Part I: Commun., Speech and Vision, vol. 138, pp. 153-161, June 1991.

[28] M. Schwartz et al., Communication Systems and Techniques. New York, NY: McGraw-Hill, 1966.

[29] M. B. Pursley and H. F. A. Roefs, "Numerical evaluation of correlation parameters for optimal phases of binary shift-register sequences," IEEE Trans. on Communications, vol. 27, pp. 1597-1604, Oct. 1979.

[30] K. G. Beauchamp, Walsh Functions and Their Applications. London: Academic Press Inc. (London) Ltd, 1975.

[31] A. J. Viterbi, CDMA: Principles of Spread Spectrum Communication. Reading, Massachusetts: Addison-Wesley Publishing Company, 1995.

[32] W. C. Y. Lee, "Overview of cellula CDMA," IEEE Transactions on Vehicular Technology, vol. 40, pp. 291-302, May 1991.

[33] G. L. Stüber and C. Kchao, "Analysis of a multiple-cell direct-sequence CDMA cellular mobile radio system," IEEE Journal on Selected Areas in Communications, vol. 10, pp. 669-679, May 1992. 
[34] S. C. Schwartz and Y. S. Yeh, "On the distribution function and moments of power sums with lognormal components," Bell Syst. Tech. J;; vol. 61', pp. 1441-1462, Sept. 1982.

[35] K. S. Gilhousen et al., "On the capacity of a cellula CDMA system," IEEE Transactions on Vehicular Technology, vol. 40, pp. 303-312, May 1991.

[36] R. J. C. Bultitude and G. C. Bedal, "Propagation characteristics on microcellular urban mobile radio channels at $910 \mathrm{MHz}$," IEEE Journal on Selected Areas in Communications, vol. 7, pp. 31-39, Jan. 1989.

[37] S. W. Golomb, Shift Register Sequences. San Francisco, California: Holden-Day Inc, 1967. 